\title{
Spatiotemporal Information Coupling in Network Navigation
}

\author{
Santiago Mazuelas, Senior Member, IEEE, Yuan Shen, Member, IEEE, and Moe Z. Win, Fellow, IEEE
}

\begin{abstract}
Network navigation, encompassing both spatial and temporal cooperation to locate mobile agents, is a key enabler for numerous emerging location-based applications. In such cooperative networks, the positional information obtained by each agent is a complex compound due to the interaction among its neighbors. This information coupling may result in poor performance: algorithms that discard information coupling are often inaccurate, and algorithms that keep track of all the neighbors' interactions are often inefficient. In this paper, we develop a principled framework to characterize the information coupling present in network navigation. Specifically, we derive the equivalent Fisher information matrix for individual agents as the sum of effective information from each neighbor and the coupled information induced by the neighbors' interaction. We further characterize how coupled information decays with the network distance in representative case studies. The results of this work can offer guidelines for the development of distributed techniques that adequately account for information coupling, and hence enable accurate and efficient network navigation.
\end{abstract}

Index Terms-Fisher information, localization, navigation, spatiotemporal cooperation, information coupling, inference.

\section{INTRODUCTION}

$\mathbf{N}$ ETWORK NAVIGATION is an emerging paradigm for providing location awareness of mobile nodes in a network with unprecedented accuracy and reliability [1]-[3]. This new paradigm will enable numerous future location-based applications such as healthcare monitoring, personnel/asset tracking, emergency evacuation, search/rescue operations, autonomous vehicles, and military operations [4]-[12]. These potential applications have motivated increasing research interest in localization and tracking technologies in the past decade [13]-[26].

Manuscript received XXXX, YYYY, and revised AAAA, BBBB

This research was supported in part by the Office of Naval Research under Grant N00014-16-1-2141, the U.S. Department of Commerce, National Institute of Standards and Technology under Grant 70NANB17H177, the MIT Institute for Soldier Nanotechnologies, the Spanish Ministry of Economy and Competitiveness MINECO under Ramon y Cajal Grant RYC-2016-19383, and the National Natural Science Foundation of China under Grant 61501279. The material in this paper was presented, in part, at the IEEE Global Communications Conference, Anaheim, CA, Dec. 2012.

S. Mazuelas was with the Wireless Information and Network Sciences Laboratory, Massachusetts Institute of Technology, Cambridge, MA 02139 USA. He is now with the BCAM-Basque Center for Applied Mathematics, and IKERBASQUE-Basque Foundation for Science, Bilbao 48009, Spain (email: smazuelas@bcamath.org).

Y. Shen was with the Wireless Information and Network Sciences Laboratory, Massachusetts Institute of Technology, Cambridge, MA 02139 USA $\mathrm{He}$ is now with the Department of Electronic Engineering, Tsinghua University, and Beijing National Research Center for Information Science and Technology, Beijing 100084, China (e-mail: shenyuan_ee@tsinghua.edu.cn).

M. Z. Win is with the Wireless Information and Network Sciences Laboratory, Massachusetts Institute of Technology, Cambridge, MA 02139 USA (e-mail: moewin@mit.edu).

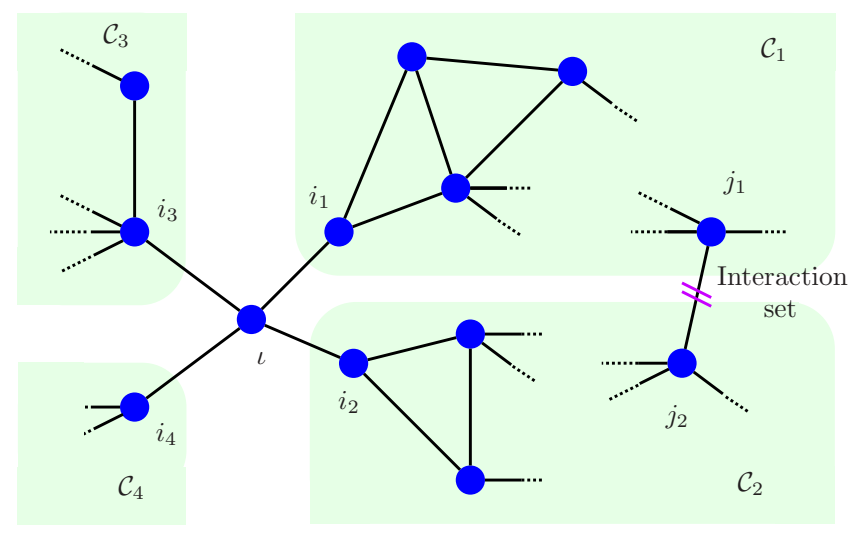

Fig. 1. A network navigation scenario where blue dots represent different states, while edges represent pairs of states that share information. Positional information for state $\iota$ is obtained from neighbors $i_{1}, i_{2}, i_{3}$, and $i_{4}$ that likewise obtain information from states in components $\mathcal{C}_{1}, \mathcal{C}_{2}, \mathcal{C}_{3}$, and $\mathcal{C}_{4}$, respectively. The double slash denotes an interaction set, as will be defined in Section

In classic localization techniques, the states of the mobile agents are inferred from the measurements between anchors (with known positions) and agents [27]-[30]. The state usually includes the agent position and possibly other mobility parameters such as velocity and acceleration, while typical measurements for localization are inter-node distances and angles-ofarrival. However, in harsh propagation environments without sufficient line-of-sight measurements between the anchors and agents, these techniques yield unsatisfactory performance or even completely fail [31]-[34]. For example, the global positioning system (GPS)-based navigation hardly works in indoor environments [35]-[37]. Other localization techniques use intra-node inertial measurements of an agent to determine its moving trajectory [38]-[42], which is commonly known as dead-reckoning. These techniques suffer from cumulative errors (positional drift) and thus lose localization accuracy over time. The above limitations have resulted in remarkable ongoing research efforts to overcome the drawbacks of harsh propagation environments for anchor-based techniques [43][46] and cumulative errors for inertial-based techniques [38][40]. However, the performance of such techniques still relies on high-density anchor deployment, high-power anchors, or high-grade inertial measurement units-none of which are cost-effective solutions - to achieve reliable and accurate ubiquitous localization.

Driven by the success in wireless communications [47][50], cooperative techniques have been introduced to improve 
the localization accuracy by sharing information and performing measurements among spatial neighbors [51]-[63]. In particular, network navigation provides a new framework in which agents exploit both spatial and temporal cooperation to infer their states [1]-[3]. Under this framework, each agent obtains information about its state from prior knowledge, inter- and intra-node measurements, and messages from neighbors (see Fig. 11. It was shown that the spatiotemporal cooperation can significantly improve the localization accuracy by providing additional information.

In terms of implementation, distributed algorithms that do not rely on a central processor are often preferred for network navigation since they can reduce communication requirements and increase system robustness $[64]-[66]$. The main difficulty for the distributed schemes lies in that the information for each state depends not only on its interaction with neighboring states but also on the interaction of those neighbors among themselves [67]-[71] 1 The interaction among neighbors causes information coupling, that is, the positional information of a state is not the sum of effective information obtained from each neighbor. Such information coupling hinders the development of efficient and accurate distributed algorithms since monitoring completely the interaction among neighbors requires a difficult network coordination.

Most current distributed algorithms either simply discard the information coupling or use ad-hoc methods to keep track of neighbors' interaction, resulting in sub-optimal performances. For instance, techniques based on belief propagation (BP) may fail to obtain accurate solutions due to the presence of cycles in the associated inference graph [51]-[53] (see also Section VI-A). In fact, loopy BP algorithms only obtain approximate beliefs since the underlying independence assumptions do not hold in general and iterative algorithms may use the same measurements repeatedly. The information coupling phenomenon has also been observed using other algorithms and some mitigation techniques have been proposed. Specifically, it was observed in [72]-[74] that ignoring the cross-correlations among positional estimates can have a negative effect, often leading to biased position estimates. Those studies explore mitigation methods that either prevent certain cooperations [53] or perform a careful bookkeeping of cross-correlations and the origins of the used data [74]. To fully unleash the potential of network navigation, the design of distributed algorithms requires a principled characterization of the information coupling due to the complex information dynamics induced by spatiotemporal cooperation.

Based on Fisher information analysis, the fundamental limits or the performance bounds of localization accuracy for network localization are derived in [55] and then the results are extended to network navigation in [2] 2 In particular, since the Fisher information matrix (FIM) for network navigation is non-diagonal [55], the equivalent FIM (EFIM) for a state

\footnotetext{
${ }^{1}$ A neighboring state refers to the state of either a spatial neighbor or a temporal neighbor, where a temporal neighbor means the same agent at a consecutive time step.

${ }^{2}$ The Fisher information is the most common metric to characterize the performance limit of inference problems in terms of the information inequality or Cramér-Rao lower bound (CRLB) 75.
}

is not the sum of effective information obtained from each neighbor, and depends also on the coupled information due to the interaction among neighbors. Recent studies analyzed the position error propagation in spatial and temporal domains for cooperative localization [68], [69], where the recursive expressions of the EFIM are obtained by approximating part of the non-diagonal terms in the FIM. To the best of the authors' knowledge, the only works that have studied information coupling are [70], [71], which focus on simple networks with three or four cooperating agents.

In this paper, we characterize the effect of information coupling arising from neighbors' interaction in general networks, and derive the closed-form expressions for the coupled information. Specifically, the main contributions of the paper are as follows.

- We derive the EFIM for individual states in navigation networks and the effective information obtained from each neighbor.

- We characterize the coupled information induced by sets of pair-wise information links and determine how information coupling decays with the network distance.

- We derive the closed-form expression of the coupled information arising from neighbors' interaction through one information link.

- We determine the effect of the information coupling in representative case studies through analytical expressions and numerical results.

- We show the impact of information coupling in the performance of distributed algorithms, and outline design guidelines for coupling-aware algorithms inspired by the theoretical results.

The rest of the paper is organized as follows. Section II describes the FIM for network navigation and the graph structure induced by the FIM in the set of states. Section III derives the EFIMs for individual states and characterizes the information coupling. Section IV specifies the expressions for coupled information in representative case studies. Section V provides numerical results for coupled information in several scenarios, and Section VI shows the relevance of the results presented for distributed algorithms. Finally, conclusions are drawn in Section VII.

Notations: The notation $[\cdot]^{\mathrm{T}}$ denotes the transpose of its argument. Random variables are shown in sans serif, upright fonts; their realizations in serif, italic fonts. Vectors are denoted by bold lowercase letters. For example, a random variable (RV) and its realization are denoted by $\mathrm{x}$ and $x$; a random vector and its realization are denoted by $\mathbf{x}$ and $\boldsymbol{x}$. The function $f_{\mathbf{x}}(\boldsymbol{x})$ and, for brevity when possible, $f(\boldsymbol{x})$ denote the probability density function (PDF) of a continuous RV $\mathbf{x}$; $f_{\mathbf{x} \mid \mathbf{y}}(\boldsymbol{x} \mid \boldsymbol{y})$ and, for brevity when possible, $f(\boldsymbol{x} \mid \boldsymbol{y})$ denote the PDF of $\mathbf{x}$ conditional on $\mathbf{y}=\boldsymbol{y} . \boldsymbol{e}_{i}^{S}$ denotes the $S$-dimensional vector with all zeros except a one at $i$ th element. $I$ denotes a generic identity matrix and $\boldsymbol{I}_{S}$ denotes a $S \times S$ identity matrix. $\otimes$ denotes the Kronecker product. $\|\boldsymbol{A}\|_{*}$ denotes the nuclear norm of a matrix $\boldsymbol{A}$. $\operatorname{diag}\left\{M_{1}, M_{2}, \ldots, M_{k}\right\}$ denotes the block diagonal matrix formed by concatenating the matrices $\boldsymbol{M}_{i}, i=1,2, \ldots, k . \mathbb{S}_{+}^{D}$ and $\mathbb{S}_{++}^{D}$ denote the set of 
$D \times D$ real positive semi-definite and positive definite matrices, respectively. For $\boldsymbol{A}, \boldsymbol{B} \in \mathbb{S}_{+}^{D}, \boldsymbol{A} \prec \boldsymbol{B}, \boldsymbol{A} \preccurlyeq \boldsymbol{B}, \boldsymbol{A} \succ \boldsymbol{B}$, and $\boldsymbol{A} \succcurlyeq \boldsymbol{B}$ denote, respectively the fact that $\boldsymbol{B}-\boldsymbol{A} \in \mathbb{S}_{++}^{D}$, $\boldsymbol{B}-\boldsymbol{A} \in \mathbb{S}_{+}^{D}, \boldsymbol{A}-\boldsymbol{B} \in \mathbb{S}_{++}^{D}$, and $\boldsymbol{A}-\boldsymbol{B} \in \mathbb{S}_{+}^{D}$.

\section{Fisher INFORMATION IN NETwork NAVIGATION}

This section describes the FIM for network navigation and the graph structure that such FIM induces in the set of states. These matrices and graph structure are used in the subsequent sections to characterize the information coupling via the EFIMs.

\section{A. Preliminaries}

Consider a cooperative network consisting of $K$ nodes. Let $\boldsymbol{x}_{k}^{(n)} \in \mathbb{R}^{D}$ be the state of node $k$ at time $t_{n}$ for $k=1,2, \ldots, K$ and $n=1,2, \ldots, N$. The goal of network navigation is to infer the states of the nodes from measurements and prior knowledge $\beta^{\beta}$ Let $\mathcal{S}=\{1,2, \ldots, S\}$ with $S=K N$ be an index set of all the states. For notational convenience, we refer to the state of node $k$ at time step $t_{n}$ either by $\boldsymbol{x}_{k}^{(n)}$ or by its index $i \in \mathcal{S}$.

It has been proven [2] that the FIM for the $S$ states can be decomposed as

$$
\boldsymbol{J}=\sum_{(i, j) \in \mathcal{S}^{2}, i \geqslant j} \boldsymbol{G}_{i, j}^{S} \otimes \boldsymbol{K}_{i, j}
$$

where

$$
\boldsymbol{G}_{i, j}^{S}= \begin{cases}\left(\boldsymbol{e}_{i}^{S}-\boldsymbol{e}_{j}^{S}\right)\left(\boldsymbol{e}_{i}^{S}-\boldsymbol{e}_{j}^{S}\right)^{\mathrm{T}}, & i \neq j \\ \boldsymbol{e}_{i}^{S}\left(\boldsymbol{e}_{i}^{S}\right)^{\mathrm{T}}, & i=j .\end{cases}
$$

The matrix $\boldsymbol{K}_{i, j} \in \mathbb{S}_{+}^{D}$ accounts for the pair-wise positional information from the measurements or prior knowledge related jointly to the states $i$ and $j$. For example, if $\mathbf{y}_{i, j}$ are the measurements related to states $\boldsymbol{x}_{i}$ and $\boldsymbol{x}_{j}, i, j \in \mathcal{S}$,

$$
\boldsymbol{K}_{i, j}=\mathbb{E}\left\{-\frac{\partial^{2} \ln f\left(\mathbf{y}_{i, j} \mid \boldsymbol{x}_{i}, \boldsymbol{x}_{j}\right)}{\partial \boldsymbol{x}_{i} \partial \boldsymbol{x}_{i}^{\mathrm{T}}}\right\} .
$$

Similarly, if $f\left(\boldsymbol{x}_{i}, \boldsymbol{x}_{j}\right)$ models the prior knowledge related to states $\boldsymbol{x}_{i}$ and $\boldsymbol{x}_{j}, i, j \in \mathcal{S}$,

$$
\boldsymbol{K}_{i, j}=\mathbb{E}\left\{-\frac{\partial^{2} \ln f\left(\boldsymbol{x}_{i}, \boldsymbol{x}_{j}\right)}{\partial \boldsymbol{x}_{i} \partial \boldsymbol{x}_{i}^{\mathrm{T}}}\right\} .
$$

As special cases, $\boldsymbol{K}_{i, j}$ is the zero matrix in the absence of such measurements or prior knowledge, and $\boldsymbol{K}_{i, i}$ accounts for the self positional information related only with state $i$, e.g., prior knowledge or information from anchors. Moreover, we assume that measurements and prior knowledge are related to the states pair-wise differences, and hence $\boldsymbol{K}_{i, j}=\boldsymbol{K}_{j, i}$.

Proposition 1: Each term $\boldsymbol{G}_{i, j}^{S} \otimes \boldsymbol{K}_{i, j}$ in (1) is a positive semidefinite matrix that has rank equal to the rank of $\boldsymbol{K}_{i, j}$. In addition, if $\boldsymbol{K}_{i, i} \succ 0$ for all $i \in \mathcal{S}$ then $\boldsymbol{J} \succ 0 \sharp$

\footnotetext{
${ }^{3}$ The state includes position and possibly other mobility parameters such as velocity, acceleration, orientation, and angular velocity; examples of measurement sensors include RF radios and inertial devices; and examples of prior knowledge include positions of certain nodes and mobility models.

${ }^{4}$ In the following, we assume $\boldsymbol{K}_{i, i} \succ 0$ for all $i \in \mathcal{S}$, while $\boldsymbol{K}_{i, j}$ for $i \neq j$ are not necessarily full rank.
}

Proof: See Appendix A

Let $\mathcal{A}$ be a subset of the natural numbers, $M$ be a matrix of size $|\mathcal{A}| D \times|\mathcal{A}| D$, so that we can associate each element in $\mathcal{A}$ with a $D \times|\mathcal{A}| D$ block-row or $|\mathcal{A}| D \times D$ block-column of $\boldsymbol{M}$. We denote by $\boldsymbol{M}_{\mathcal{A}_{1}, \mathcal{A}_{2}}$ the sub-matrix of $M$ formed by the blocks corresponding to rows associated with $\mathcal{A}_{1}$ and columns associated with $\mathcal{A}_{2}$ for $\mathcal{A}_{1}, \mathcal{A}_{2} \subseteq \mathcal{A}$. For instance, in a navigation network with three states, $\bar{J}_{\{1\},\{2,3\}}$ denotes the upper right $D \times 2 D$ block of the FIM.

In the example of Fig. 1, the FIM for all the states can be written as

$$
\boldsymbol{J}=\left[\begin{array}{ccccc}
\boldsymbol{J}_{\iota, \iota} & \boldsymbol{J}_{\iota, \mathcal{C}_{1}} & \boldsymbol{J}_{\iota, \mathcal{C}_{2}} & \boldsymbol{J}_{\iota, \mathcal{C}_{3}} & \boldsymbol{J}_{\iota, \mathcal{C}_{4}} \\
\boldsymbol{J}_{\mathcal{C}_{1}, \iota} & \boldsymbol{J}_{\mathcal{C}_{1}, \mathcal{C}_{1}} & \boldsymbol{J}_{\mathcal{C}_{1}, \mathcal{C}_{2}} & 0 & 0 \\
\boldsymbol{J}_{\mathcal{C}_{2}, \iota} & \boldsymbol{J}_{\mathcal{C}_{2}, \mathcal{C}_{1}} & \boldsymbol{J}_{\mathcal{C}_{2}, \mathcal{C}_{2}} & 0 & 0 \\
\boldsymbol{J}_{\mathcal{C}_{3}, \iota} & 0 & 0 & \boldsymbol{J}_{\mathcal{C}_{3}, \mathcal{C}_{3}} & 0 \\
\boldsymbol{J}_{\mathcal{C}_{4}, \iota} & 0 & 0 & 0 & \boldsymbol{J}_{\mathcal{C}_{4}, \mathcal{C}_{4}}
\end{array}\right]
$$

where

$$
\begin{aligned}
\boldsymbol{J}_{\iota, \iota} & =\boldsymbol{K}_{\iota, \iota}+\sum_{k=1}^{4} \boldsymbol{K}_{\iota, i_{k}} \\
\boldsymbol{J}_{\iota, \mathcal{C}_{k}} & =\boldsymbol{J}_{\mathcal{C}_{k}, \iota}^{\mathrm{T}}=-\left[\begin{array}{llll}
\boldsymbol{K}_{\iota, i_{k}} & 0 & \cdots & 0
\end{array}\right]
\end{aligned}
$$

for $k=1,2,3,4$, and

$$
\begin{aligned}
{\left[\boldsymbol{J}_{\mathcal{C}_{1}, \mathcal{C}_{2}}\right]_{j_{1}, j_{2}} } & =\left[\boldsymbol{J}_{\mathcal{C}_{2}, \mathcal{C}_{1}}\right]_{j_{2}, j_{1}}=-\boldsymbol{K}_{j_{1}, j_{2}} \\
{\left[\boldsymbol{J}_{\mathcal{C}_{1}, \mathcal{C}_{2}}\right]_{i, j} } & =\left[\boldsymbol{J}_{\mathcal{C}_{2}, \mathcal{C}_{1}}\right]_{j, i}=0
\end{aligned}
$$

for $(i, j) \neq\left(j_{1}, j_{2}\right)$.

Based on the structure of the FIM, we next introduce a graph structure for the set of states, which will be used to determine the interrelationship among the different information components.

\section{B. Structure of the States from FIM}

The FIM induces a graph-structure for the set of states that we refer to as the navigation information graph (NIG). Its vertices correspond to the states, and its edges (links) correspond to the pairs of states $(i, j) \in \mathcal{S}^{2}$ for which $\boldsymbol{K}_{i, j} \neq 0$ (see Fig. 1) 5 Based on the terminologies in graph theory, we next give several definitions for the NIG.

Definition 1: The matrix $\boldsymbol{G}_{i, j}^{S} \otimes \boldsymbol{K}_{i, j}$ is the information link related to the states $i$ and $j$ for $(i, j) \in \mathcal{S}^{2}$, and the matrix $\boldsymbol{K}_{i, j} \in \mathbb{S}_{+}^{D}$ is the capacity of the link. Two states $i \neq j$ are neighbors if $\boldsymbol{K}_{i, j} \neq 0$, and $\mathcal{N}_{i}$ denotes the set of states that are neighbors of state $i$.

Definition 2: Two states $i$ and $j$ are connected in a set of states $\overline{\mathcal{S}} \subset \mathcal{S}$ if there exists a path between $i$ and $j$ in $\overline{\mathcal{S}}$, i.e., a sequence of states $\left\{k_{1}, k_{2}, \ldots, k_{r+1}\right\} \subset \overline{\mathcal{S}}$ with $k_{1}=i$ and $k_{r+1}=j$ such that $\boldsymbol{K}_{k_{s}, k_{s}+1} \neq 0$ for $s=1,2, \ldots, r$. The network distance between two connected states $i$ and $j$, denoted by $\mathrm{ND}(i, j)$, is the length of the shortest path between $i$ and $j$. A connected component of $\overline{\mathcal{S}}$ is a maximal subset of states connected in $\overline{\mathcal{S}}$.

In this paper, without loss of generality we focus on a specific state $\iota \in \mathcal{S}$. The set $\overline{\mathcal{S}}=\mathcal{S} \backslash\{\iota\}$ can be uniquely

\footnotetext{
${ }^{5}$ Notice that the FIM in 11 corresponds to the Laplacian of the undirected graph with vertices corresponding to the states and edges weighted by the semidefinite matrices $\boldsymbol{K}_{i, j}[76$.
} 
partitioned as the disjoint union of connected components. In the network example shown in Fig. 11 $\overline{\mathcal{S}}$ has three connected components (i.e., $\left(\mathcal{C}_{1} \cup \mathcal{C}_{2}\right), \mathcal{C}_{3}$, and $\left.\mathcal{C}_{4}\right)$ if $\boldsymbol{K}_{j_{1}, j_{2}} \neq 0$ and four connected components (i.e., $\mathcal{C}_{1}, \mathcal{C}_{2}, \mathcal{C}_{3}$, and $\mathcal{C}_{4}$ ) if $\boldsymbol{K}_{j_{1}, j_{2}}=0$. In the former case the component $\left(\mathcal{C}_{1} \cup \mathcal{C}_{2}\right)$ contains two neighbors of $\iota$, and in the latter case each component contains only one neighbor of $\iota$.

In the next section, we characterize the effective information obtained from each neighbor as well as the coupled information due to neighbors' interaction.

\section{INFORMATION COUPLING IN NETWORK NAVIGATION}

This section first derives the EFIM for individual states and characterizes the effect of the information coupling induced by sets of information links. Then, we determine the decay of information coupling with the network distance and the closed-form EFIM for networks coupled by one information link.

\section{A. EFIM for Individual States}

The FIM $\boldsymbol{J}$ in (1) captures the information for all the states in $\mathcal{S}$, and thus determines the performance bound for joint estimation of the states. To further study information coupling, we will next adopt the notion of the EFIM [32]. Such matrix represents the equivalent information for states' subsets and shows how coupled information arises as a consequence of other states' interaction.

Definition 3 (EFIM [32]): Given a parameter vector $\boldsymbol{\theta}=$ $\left[\begin{array}{ll}\boldsymbol{\theta}_{1}^{\mathrm{T}} & \boldsymbol{\theta}_{2}^{\mathrm{T}}\end{array}\right]^{\mathrm{T}}$ and the FIM $\boldsymbol{J}_{\boldsymbol{\theta}}$ of the form

$$
J_{\theta}=\left[\begin{array}{cc}
A & B \\
B^{\mathrm{T}} & C
\end{array}\right]
$$

where $\boldsymbol{\theta} \in \mathbb{R}^{N}, \boldsymbol{\theta}_{1} \in \mathbb{R}^{n}, \boldsymbol{A} \in \mathbb{R}^{n \times n}, \boldsymbol{B} \in \mathbb{R}^{n \times(N-n)}$, and $\boldsymbol{C} \in \mathbb{R}^{(N-n) \times(N-n)}$ with $1 \leqslant n<N$, the EFIM for $\boldsymbol{\theta}_{1}$ is given by

$$
J_{\mathrm{e}}\left(\boldsymbol{\theta}_{1}\right):=\boldsymbol{A}-\boldsymbol{B} \boldsymbol{C}^{-1} \boldsymbol{B}^{\mathrm{T}} .
$$

Note that the right-hand side of 9 is known as the Schur complement of block-matrix $\boldsymbol{A}$ in matrix $\boldsymbol{J}_{\boldsymbol{\theta}}$ [77]. It is clear that the EFIM retains all the necessary information to derive the information inequality for the parameter $\boldsymbol{\theta}_{1}$. In other words, the CRLB for $\boldsymbol{\theta}_{1}$ can be obtained by the inverse of $J_{\mathrm{e}}\left(\boldsymbol{\theta}_{1}\right)$ rather than directly inverting a high-dimensional matrix $\boldsymbol{J}_{\boldsymbol{\theta}}$. More importantly, the use of the EFIM allows to characterize all the information components of each individual state, which is essential for studying the effect of information coupling.

The following theorem shows that the EFIM $J_{\mathrm{e}}$ for a generic state $\iota \in \mathcal{S}$ can be decomposed as a sum of terms, each corresponding to a connected component of $\overline{\mathcal{S}}$ that contains neighbors of $\iota$.

Theorem 1: Let $\overline{\mathcal{S}}=\mathcal{C}_{1} \cup \mathcal{C}_{2} \cup \ldots \cup \mathcal{C}_{n}$ be a disjoint partition of $\overline{\mathcal{S}}$ as a union of connected components, where only the first $m$ components have nonempty intersection with $\mathcal{N}_{\iota}$, i.e., $\mathcal{C}_{k} \cap \mathcal{N}_{\iota}=\varnothing$ for $m<k \leqslant n$. Then the EFIM for state $\iota$ is

$$
\boldsymbol{J}_{\mathrm{e}}=\boldsymbol{K}_{\iota, \iota}+\sum_{k=1}^{m}\left[\sum_{i \in \mathcal{N}_{\iota} \cap \mathcal{C}_{k}} \boldsymbol{K}_{\iota, i}-\boldsymbol{J}_{\iota, \mathcal{N}_{\iota} \cap \mathcal{C}_{k}} \boldsymbol{\Lambda}_{k} \boldsymbol{J}_{\iota, \mathcal{N}_{\iota} \cap \mathcal{C}_{k}}^{\mathrm{T}}\right]
$$

where $\boldsymbol{\Lambda}_{k}=\left[\boldsymbol{J}_{\mathcal{C}_{k}, \mathcal{C}_{k}}^{-1}\right]_{\mathcal{N}_{\iota} \cap \mathcal{C}_{k}, \mathcal{N}_{\iota} \cap \mathcal{C}_{k}}$.

Proof: From the definition of the EFIM, we have that

$$
\begin{aligned}
& \boldsymbol{J}_{\mathrm{e}}=\boldsymbol{K}_{\iota, \iota}+\sum_{i \in \mathcal{N}_{\iota}} \boldsymbol{K}_{\iota, i}-\left[\begin{array}{llll}
\boldsymbol{J}_{\iota, \mathcal{C}_{1}} & \boldsymbol{J}_{\iota, \mathcal{C}_{2}} & \cdots & \boldsymbol{J}_{\iota, \mathcal{C}_{n}}
\end{array}\right] \\
& \cdot \boldsymbol{J}_{\overline{\mathcal{S}}, \overline{\mathcal{S}}}^{-1}\left[\begin{array}{llll}
\boldsymbol{J}_{\iota, \mathcal{C}_{1}} & \boldsymbol{J}_{\iota, \mathcal{C}_{2}} & \cdots & \boldsymbol{J}_{\iota, \mathcal{C}_{n}}
\end{array}\right]^{\mathrm{T}} \\
& =\boldsymbol{K}_{\iota, \iota}+\sum_{i \in \mathcal{N}_{\iota}} \boldsymbol{K}_{\iota, i}-\left[\begin{array}{llll}
\boldsymbol{J}_{\iota, \mathcal{C}_{1}} & \boldsymbol{J}_{\iota, \mathcal{C}_{2}} & \cdots & \boldsymbol{J}_{\iota, \mathcal{C}_{m}}
\end{array}\right] \\
& \cdot \operatorname{diag}\left\{\boldsymbol{J}_{\mathcal{C}_{1}, \mathcal{C}_{1}}^{-1}, \boldsymbol{J}_{\mathcal{C}_{2}, \mathcal{C}_{2}}^{-1}, \ldots, \boldsymbol{J}_{\mathcal{C}_{m}, \mathcal{C}_{m}}^{-1}\right\} \\
& \cdot\left[\begin{array}{llll}
\boldsymbol{J}_{\iota, \mathcal{C}_{1}} & \boldsymbol{J}_{\iota, \mathcal{C}_{2}} & \cdots & \boldsymbol{J}_{\iota, \mathcal{C}_{m}}
\end{array}\right]^{\mathrm{T}} \\
& =\boldsymbol{K}_{\iota, \iota}+\sum_{i \in \mathcal{N}_{\iota}} \boldsymbol{K}_{\iota, i}-\sum_{k=1}^{m} \boldsymbol{J}_{\iota, \mathcal{C}_{k}} \boldsymbol{J}_{\mathcal{C}_{k}, \mathcal{C}_{k}}^{-1} \boldsymbol{J}_{\iota, \mathcal{C}_{k}}^{\mathrm{T}}
\end{aligned}
$$

where the second equality is due to

$$
\boldsymbol{J}_{\overline{\mathcal{S}}, \overline{\mathcal{S}}}=\operatorname{diag}\left\{\boldsymbol{J}_{\mathcal{C}_{1}, \mathcal{C}_{1}}, \boldsymbol{J}_{\mathcal{C}_{2}, \mathcal{C}_{2}}, \ldots, \boldsymbol{J}_{\mathcal{C}_{n}, \mathcal{C}_{n}}\right\}
$$

and $\boldsymbol{J}_{\iota, \mathcal{C}_{i}}=0$ for $i>m$. The result 10) is obtained by observing that

$$
\boldsymbol{J}_{\iota, \mathcal{C}_{k}} \boldsymbol{J}_{\mathcal{C}_{k}, \mathcal{C}_{k}}^{-1} \boldsymbol{J}_{\iota, \mathcal{C}_{k}}^{\mathrm{T}}=\boldsymbol{J}_{\iota, \mathcal{N}_{\iota} \cap \mathcal{C}_{k}} \boldsymbol{\Lambda}_{k} \boldsymbol{J}_{\iota, \mathcal{N}_{\iota} \cap \mathcal{C}_{k}}^{\mathrm{T}}
$$

and that the non-zero blocks of $\boldsymbol{J}_{\iota, \mathcal{C}_{k}}$ are those corresponding to $\boldsymbol{J}_{\iota, \mathcal{N}_{\iota} \cap \mathcal{C}_{k}}$.

The theorem shows that the information obtained by state $\iota$ is the sum of the self positional information and that obtained from each connected component, where the latter is the difference between the capacity of the links to the neighbors in such a component and a term that accounts for the uncertainty of neighbors in the component.

Definition 4: The neighbors of a state $\iota \in \mathcal{S}$ in $\mathcal{N}_{\iota}$ are isolated if each connected component of $\overline{\mathcal{S}}$ contains at most one neighbor of $\iota$, i.e., for each connected component $\mathcal{C}_{k}, \mid C_{k} \cap$ $\mathcal{N}_{\iota} \mid \leqslant 1$.

In the network example in Fig. 1, the neighbors of state $\iota$ are isolated if $\boldsymbol{K}_{j_{1}, j_{2}}=0$. The following result shows that when the neighbors are isolated, the information obtained through cooperation can be decoupled as the sum of the information obtained from each neighbor.

Corollary 1: If the neighbors of state $\iota$ are isolated, then the EFIM for state $\iota$ is

$$
\boldsymbol{J}_{\mathrm{e}}=\boldsymbol{K}_{\iota, \iota}+\sum_{i \in \mathcal{N}_{\iota}}\left(\boldsymbol{I}-\boldsymbol{K}_{\iota, i} \boldsymbol{\Lambda}_{k_{i}}\right) \boldsymbol{K}_{\iota, i}
$$

where $k_{i}$ is the index of the component corresponding to neighbor $i$, i.e., $\mathcal{C}_{k_{i}} \cap \mathcal{N}_{\iota}=\{i\}$.

Proof: The proof is a direct consequence of Theorem 1

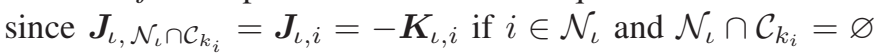
in other case.

This result shows that when the neighbors are isolated, the information obtained through cooperation is the sum of the information obtained from each neighbor. In addition, each of 
those terms depends on the capacity of the link to the neighbor, i.e., $\boldsymbol{K}_{\iota, i}$, and on the neighbor's uncertainty induced by its component, i.e., $\boldsymbol{\Lambda}_{k_{i}}$. Such amenable situation characterizes uncoupled neighbors.

Definition 5: The neighbors of state $\iota \in \mathcal{S}$ in $\mathcal{N}_{\iota}$ are uncoupled if

$$
\boldsymbol{J}_{\mathrm{e}}=\boldsymbol{K}_{\iota, \iota}+\sum_{i \in \mathcal{N}_{\iota}} \boldsymbol{K}_{i \rightarrow \iota}
$$

with

$$
\boldsymbol{K}_{i \rightarrow \iota} \triangleq\left(\boldsymbol{I}-\boldsymbol{K}_{\iota, i} \boldsymbol{\Lambda}_{k_{i}}\right) \boldsymbol{K}_{\iota, i}
$$

representing the effective information that can be obtained from neighbor $i$ taking into account its own uncertainty. In addition, we say that a state is subject to information coupling when its neighbors are not uncoupled.

\section{B. Information Coupling and Effective Information}

The results below further characterize the terms corresponding to the information that can be effectively obtained from each neighbor as well as the information coupling.

Definition 6: Let $\mathcal{B}$ be a set of links between the states, the $\mathcal{B}$-reduced FIM is defined as

$$
\begin{aligned}
\boldsymbol{J}^{\mathcal{B}} & =\sum_{(i, j) \in \mathcal{S}^{2} \backslash \mathcal{B}, i \geqslant j} \boldsymbol{G}_{i, j}^{S} \otimes \boldsymbol{K}_{i, j} \\
& =\boldsymbol{J}-\sum_{(i, j) \in \mathcal{B}, i \geqslant j} \boldsymbol{G}_{i, j}^{S} \otimes \boldsymbol{K}_{i, j} .
\end{aligned}
$$

Then, the $\mathcal{B}$-effective link information from $i \in \mathcal{N}_{\iota}$ to $\iota$ is defined as

$$
\boldsymbol{K}_{i \rightarrow \iota}^{\mathcal{B}}=\left(\boldsymbol{I}-\boldsymbol{K}_{\iota, i} \boldsymbol{\Lambda}_{k_{i}}^{\mathcal{B}}\right) \boldsymbol{K}_{\iota, i}
$$

where

$$
\boldsymbol{\Lambda}_{k_{i}}^{\mathcal{B}}=\left[\left(\boldsymbol{J}_{\mathcal{C}_{k_{i}}, \mathcal{C}_{k_{i}}}^{\mathcal{B}}\right)^{-1}\right]_{i, i}
$$

and $i \in \mathcal{C}_{k_{i}} \cap \mathcal{N}_{\iota}$. Moreover, $\boldsymbol{K}_{i \rightarrow \iota}=\boldsymbol{K}_{i \rightarrow \iota}^{\mathcal{B}}$ when $\mathcal{B}=\varnothing$.

Definition 7: A set of links between the states in $\overline{\mathcal{S}}, \mathcal{B} \subset \overline{\mathcal{S}}^{2}$, is an interaction set for state $\iota$ if the neighbors of state $\iota$ are isolated for the FIM $\boldsymbol{J}^{\mathcal{B}}$ but not for the FIM $\boldsymbol{J}$.

Remark 1: This definition characterizes the sets of links such that their removal isolates the neighbors of a state, since the matrix $\boldsymbol{J}^{\mathcal{B}}$ corresponds with the FIM for $\mathcal{S}$ when $\boldsymbol{K}_{j_{1}, j_{2}}=0$ for all $\left(j_{1}, j_{2}\right) \in \mathcal{B}$ [see [17]]. For instance, in the network shown in Fig. 1 the set $\left(j_{1}, j_{2}\right)$ is an interaction set containing a single link.

Proposition 2: Let $\mathcal{B}$ be an interaction set for state $\iota$. The EFIM for state $\iota$ is bounded as

$$
\boldsymbol{K}_{\iota, \iota}+\sum_{i \in \mathcal{N}_{\iota}} \boldsymbol{K}_{i \rightarrow \iota}^{\mathcal{B}} \preccurlyeq J_{\mathrm{e}} \preccurlyeq \boldsymbol{K}_{\iota, \iota}+\sum_{i \in \mathcal{N}_{\iota}} \boldsymbol{K}_{\iota, i} .
$$

Proof: The first inequality is a consequence of Corollary 1 since $\boldsymbol{J} \succcurlyeq \boldsymbol{J}^{\mathcal{B}}$ implies that $\boldsymbol{\Lambda}_{k} \preccurlyeq \boldsymbol{\Lambda}_{k}^{\mathcal{B}}$ for any $k$. The second inequality is a direct consequence of Theorem 1 since the matrices $\boldsymbol{\Lambda}_{k}$ are positive definite.

Proposition 2 shows that the information obtained from cooperation by each state is upper bounded by the sum of the capacities of the neighboring links and lower bounded by the sum of the $\mathcal{B}$-effective information from neighbors for any interaction set $\mathcal{B}$. Moreover, such lower bound becomes an equality for $\mathcal{B}=\varnothing$ if the neighbors of state $\iota$ are isolated. Note that the effective information for cooperative localization was first introduced in [55], $\mathcal{B}$-effective information proposed in this paper is a generalization of that in [55], which corresponds to the specific interaction set $\mathcal{B}=\left\{(i, j) \in(\mathcal{S} \backslash\{\iota\})^{2}\right.$ for $i>$ $j\}$.

Remark 2: Proposition 2 has the following operational meaning: discarding several measurements or prior knowledges, i.e., letting several link capacities $\boldsymbol{K}_{i, j}=0$, decouples the neighbors of state $\iota$. Although such an operation reduces the total information for state $\iota$, it decomposes the information for state $\iota$ as a sum of that from each neighbor, which is amenable for distributed algorithms.

The next proposition shows that the effective information is always smaller than or equal to the link capacity and that the effective information increases when the interaction set $\mathcal{B}$ decreases.

Proposition 3:

1) If $\mathcal{B}$ and $\mathcal{B}^{\prime}$ are two sets of links between the states with $\mathcal{B} \subseteq \mathcal{B}^{\prime}$, then for any $i \in \mathcal{N}_{\iota}$

$$
0 \preccurlyeq \boldsymbol{K}_{i \rightarrow \iota}^{\mathcal{B}^{\prime}} \preccurlyeq \boldsymbol{K}_{i \rightarrow \iota}^{\mathcal{B}} \preccurlyeq \boldsymbol{K}_{i \rightarrow \iota} \preccurlyeq \boldsymbol{K}_{\iota, i} .
$$

2) If $\boldsymbol{K}_{i, i}=\xi_{i} \boldsymbol{I}$, for any set of links $\mathcal{B}$

$$
\lim _{\xi_{i} \rightarrow \infty} \boldsymbol{K}_{i \rightarrow \iota}^{\mathcal{B}}=\boldsymbol{K}_{\iota, i} .
$$

Proof: See Appendix C

Proposition 4: If $\boldsymbol{K}_{\iota, i}$ is rank-one, i.e., $\boldsymbol{K}_{\iota, i}=\boldsymbol{v}_{\iota, i} \boldsymbol{v}_{\iota, i}^{\mathrm{T}}$ for $\boldsymbol{v}_{\iota, i} \in \mathbb{R}^{D}$, the $\mathcal{B}$-effective link information $\boldsymbol{K}_{i \rightarrow \iota}^{\mathcal{B}}$ is proportional to $\boldsymbol{K}_{\iota, i}, \boldsymbol{K}_{i \rightarrow \iota}^{\mathcal{B}}=\xi_{\iota, i}^{\mathcal{B}} \boldsymbol{K}_{\iota, i}$ with $0<\xi_{\iota, i}^{\mathcal{B}}<1$ for any set $\mathcal{B}$, and given by

$$
\xi_{\iota, i}^{\mathcal{B}}=1-\boldsymbol{v}_{\iota, i}^{\mathrm{T}} \boldsymbol{\Lambda}_{k_{i}}^{\mathcal{B}} \boldsymbol{v}_{\iota, i}
$$

Proof: Using the definition of effective link information, we have that

$$
\begin{aligned}
\boldsymbol{K}_{i \rightarrow \iota}^{\mathcal{B}} & =\left(\boldsymbol{I}-\boldsymbol{v}_{\iota, i} \boldsymbol{v}_{\iota, i}^{\mathrm{T}} \boldsymbol{\Lambda}_{k_{i}}^{\mathcal{B}}\right) \boldsymbol{v}_{\iota, i} \boldsymbol{v}_{\iota, i}^{\mathrm{T}} \\
& =\left(1-\boldsymbol{v}_{\iota, i}^{\mathrm{T}} \boldsymbol{\Lambda}_{k_{i}}^{\mathcal{B}} \boldsymbol{v}_{\iota, i}\right) \boldsymbol{K}_{\iota, i} .
\end{aligned}
$$

The remaining part of the corollary, i.e., $\xi_{\iota, i}^{\mathcal{B}}>0$, is proven using the Lemma 1 in Appendix $\mathbb{B}$ with $\boldsymbol{C}=\boldsymbol{J}_{\mathcal{C}_{k_{i}}, \mathcal{C}_{k_{i}}}^{\mathcal{B}}$ and $\boldsymbol{A}=\boldsymbol{v}_{\iota, i} \boldsymbol{v}_{\iota, i}^{\mathrm{T}}+\boldsymbol{K}_{i, i}$. Then, the matrix $\left[\left(\boldsymbol{J}_{\mathcal{C}_{k_{i}}, \mathcal{C}_{k_{i}}}^{\mathcal{B}}\right)^{-1}\right]_{i, i}$ can be written as $\left(\boldsymbol{v}_{\iota, i} \boldsymbol{v}_{\iota, i}^{\mathrm{T}}+\boldsymbol{K}_{i, i}+\boldsymbol{R}\right)^{-1}$ with $\boldsymbol{K}_{i, i}+\boldsymbol{R} \succ 0$ for some matrix $\boldsymbol{R}$. Hence, the result is obtained since

$$
\xi_{\iota, i}^{\mathcal{B}}=\left(1+\boldsymbol{v}_{\iota, i}^{\mathrm{T}}\left(\boldsymbol{K}_{i, i}+\boldsymbol{R}\right)^{-1} \boldsymbol{v}_{\iota, i}\right)^{-1}
$$

by using the matrix inversion lemma.

Remark 3: The effective information a state obtains from a neighbor is a positive semidefinite matrix smaller than or equal to the link capacity, and it is proportional to such capacity if it is a rank-one matrix. In addition the $\mathcal{B}$-effective information increases with smaller sets $\mathcal{B}$. Finally, the effective information equals the link capacity when the self positional information of the neighbor tends to infinity 6

\footnotetext{
${ }^{6}$ We refer to the matrices $\boldsymbol{K}_{i, j}$ as link capacities since they play a similar role to the capacities of network flows as upper bounds of flows through links 78.
} 
The next definition quantifies the information coupling induced by an interaction set as the difference between the actual EFIM and the sum of effective information from each neighbor.

Definition 8: Let $\mathcal{B}$ be an interaction set for state $\iota$, the coupled information for state $\iota$ induced by the links $\mathcal{B}$ is the positive semidefinite matrix

$$
\boldsymbol{\Xi}^{\mathcal{B}}=\boldsymbol{J}_{\mathrm{e}}-\boldsymbol{J}_{\mathrm{e}}^{\mathcal{B}}=\boldsymbol{J}_{\mathrm{e}}-\boldsymbol{K}_{\iota, \iota}-\sum_{i \in \mathcal{N}_{\iota}} \boldsymbol{K}_{i \rightarrow \iota}^{\mathcal{B}} \succcurlyeq 0 .
$$

where $\boldsymbol{J}_{\mathrm{e}}^{\mathcal{B}}$ is the EFIM for state $\iota$ based on the $\mathcal{B}$-reduced FIM $J^{\mathcal{B}}$.

\section{Information Coupling Decay with Network Distance}

This section shows how the information induced by a link changes with the network distance. These results will be used in Section IV-D to show the exponential decay of information coupling with the network distance in a simple network. Here, the first result shows the difference in the EFIM, caused by the presence of a specific link, for states with increasing network distance to such link. The second result shows how the EFIM difference decreases with the network distance.

We consider subsets of the states with increasing network distance to a link $\mathcal{B}=\left\{\left(j_{1}, j_{2}\right)\right\}$. Specifically, $\mathcal{S}_{k}$ is the set of the states at network distance at least $k$ to $j_{1}$ and $j_{2}$, and $\mathcal{E}_{k}$ is the set of the states in $\mathcal{S}_{k}$ at network distance $k$ to $j_{1}$ or $j_{2}$, i.e.,

$$
\begin{aligned}
& \mathcal{S}_{k}=\left\{i \in \mathcal{S}: \operatorname{ND}\left(i, j_{1}\right) \geqslant k, \mathrm{ND}\left(i, j_{2}\right) \geqslant k\right\} \\
& \mathcal{E}_{k}=\left\{i \in \mathcal{S}: \min \left\{\mathrm{ND}\left(i, j_{1}\right), \mathrm{ND}\left(i, j_{2}\right)\right\}=k\right\} .
\end{aligned}
$$

The EFIM difference due to information link $\mathcal{B}$ is given in the following theorem.

Theorem 2: Let $\boldsymbol{J}_{k}$ and $\boldsymbol{J}_{k}^{\mathcal{B}}$ be the EFIMs of states $\mathcal{S}_{k}$ for $\boldsymbol{J}$ and $\boldsymbol{J}^{\mathcal{B}}$, respectively. Then

$$
\left[\boldsymbol{J}_{k}\right]_{i, j}= \begin{cases}{\left[\boldsymbol{J}_{k}^{\mathcal{B}}\right]_{i, j}} & \text { if }(i, j) \notin \mathcal{E}_{k}^{2} \\ {\left[\boldsymbol{J}_{k}^{\mathcal{B}}\right]_{i, j}+\left[\boldsymbol{D}_{k}\right]_{i, j}} & \text { if }(i, j) \in \mathcal{E}_{k}^{2}\end{cases}
$$

with

$$
\begin{aligned}
\boldsymbol{D}_{k} & =\boldsymbol{\Gamma}_{k} \boldsymbol{U}_{j_{1}, j_{2}}\left(\boldsymbol{I}+\boldsymbol{U}_{j_{1}, j_{2}}^{\mathrm{T}} \boldsymbol{\Delta}_{k} \boldsymbol{U}_{j_{1}, j_{2}}\right)^{-1} \boldsymbol{U}_{j_{1}, j_{2}}^{\mathrm{T}} \boldsymbol{\Gamma}_{k}^{\mathrm{T}} \\
& \preccurlyeq \boldsymbol{\Gamma}_{k} \boldsymbol{K}_{j_{1}, j_{2}} \boldsymbol{\Gamma}_{k}^{\mathrm{T}}
\end{aligned}
$$

where $\boldsymbol{U}_{j_{1}, j_{2}}$ is a square root of $\boldsymbol{K}_{j_{1}, j_{2}}$, i.e., $\boldsymbol{K}_{j_{1}, j_{2}}=$ $\boldsymbol{U}_{j_{1}, j_{2}} \boldsymbol{U}_{j_{1}, j_{2}}^{\mathrm{T}}, \boldsymbol{\Gamma}_{0}=[\boldsymbol{I},-\boldsymbol{I}]^{\mathrm{T}}, \boldsymbol{\Delta}_{0}=0$, and

$$
\begin{aligned}
\boldsymbol{\Gamma}_{k} & =\boldsymbol{J}_{\mathcal{E}_{k}, \mathcal{E}_{k-1}}\left(\left[\boldsymbol{J}_{k-1}^{\mathcal{B}}\right]_{\mathcal{E}_{k-1}, \mathcal{E}_{k-1}}\right)^{-1} \boldsymbol{\Gamma}_{k-1} \\
\boldsymbol{\Delta}_{k} & =\boldsymbol{\Delta}_{k-1}+\boldsymbol{\Gamma}_{k-1}^{\mathrm{T}}\left(\left[\boldsymbol{J}_{k-1}^{\mathcal{B}}\right]_{\mathcal{E}_{k-1}, \mathcal{E}_{k-1}}\right)^{-1} \boldsymbol{\Gamma}_{k-1}
\end{aligned}
$$

for $k=1,2, \ldots, n$, with $n$ being the largest integer for which $\mathcal{S}_{n} \neq \varnothing$.

Proof: See Appendix D.

Remark 4: Theorem 2 shows that the difference between the EFIMs with and without a specific link $\left(j_{1}, j_{2}\right)$ depends on the link capacity $\boldsymbol{K}_{j_{1}, j_{2}}$ and the network distance $k$ to the link $\left(j_{1}, j_{2}\right)$ through the recursively defined matrices $\boldsymbol{\Gamma}_{k}$.

The following result shows that the difference between EFIMs for a network distance $k+1$ is smaller than the difference between EFIMs for a network distance $k$ multiplied by the matrices that define the recurrence for $\boldsymbol{\Gamma}_{k}$.

Corollary 2: Under the conditions of Theorem 2, we have that for $k=0,1, \ldots, n$

$$
\boldsymbol{D}_{k+1} \preccurlyeq \boldsymbol{J}_{\mathcal{E}_{k+1}, \mathcal{E}_{k}}\left(\left[\boldsymbol{J}_{k}^{\mathcal{B}}\right]_{\mathcal{E}_{k}, \mathcal{E}_{k}}\right)^{-1} \boldsymbol{D}_{k}\left(\left[\boldsymbol{J}_{k}^{\mathcal{B}}\right]_{\mathcal{E}_{k}, \mathcal{E}_{k}}\right)^{-1} \boldsymbol{J}_{\mathcal{E}_{k}, \mathcal{E}_{k+1}}
$$

Proof: The result is a straightforward consequence of the previous result taking into account that

$$
\left(\boldsymbol{I}+\boldsymbol{U}_{j_{1}, j_{2}}^{\mathrm{T}} \boldsymbol{\Delta}_{k+1} \boldsymbol{U}_{j_{1}, j_{2}}\right)^{-1} \preccurlyeq\left(\boldsymbol{I}+\boldsymbol{U}_{j_{1}, j_{2}}^{\mathrm{T}} \boldsymbol{\Delta}_{k} \boldsymbol{U}_{j_{1}, j_{2}}\right)^{-1}
$$

since $\boldsymbol{\Delta}_{k} \preccurlyeq \boldsymbol{\Delta}_{k+1}$.

These two results illustrate how the information induced by sets of links decay with the network distance in the network. The next section further explores information coupling for networks where there is one coupling link.

\section{Information Coupling for One-link Coupled Neighbors}

The detailed closed-form expression of the EFIM for general networks is too complex. We present such EFIM for the case where there is an interaction set formed by one link.

Theorem 3: Let $\mathcal{B}=\left\{\left(j_{1}, j_{2}\right)\right\}$ be an interaction set for state $\iota$ and $\mathcal{C}_{1}, \mathcal{C}_{2}, \ldots, \mathcal{C}_{n}$ be a partition of $\overline{\mathcal{S}}$ into connected components for the structure induced by the $\mathcal{B}$-reduced FIM $\boldsymbol{J}^{\mathcal{B}}$. If $\left\{i_{1}, i_{2}\right\} \subseteq \mathcal{N}_{\iota}$ with $i_{1}, j_{1} \in \mathcal{C}_{1}$ and $i_{2}, j_{2} \in \mathcal{C}_{2}$, then the EFIM for state $\iota$ is

$$
\boldsymbol{J}_{\mathrm{e}}=\boldsymbol{K}_{\iota, \iota}+\sum_{i \in \mathcal{N}_{\iota}} \boldsymbol{K}_{i \rightarrow \iota}^{\mathcal{B}}+\boldsymbol{\Xi}_{i_{1}, i_{2}}^{\left(j_{1}, j_{2}\right)}
$$

where

$$
\boldsymbol{\Xi}_{i_{1}, i_{2}}^{\left(j_{1}, j_{2}\right)}=\boldsymbol{\Gamma}\left[\boldsymbol{K}\left(\boldsymbol{\Phi}^{-1}+\boldsymbol{K}\right)^{-1} \boldsymbol{\Phi}^{-1}\right] \boldsymbol{\Gamma}^{\mathrm{T}}
$$

with

$$
\begin{aligned}
\boldsymbol{K} & =\boldsymbol{K}_{j_{1}, j_{2}} \\
\boldsymbol{\Phi} & =\left[\left(\boldsymbol{J}_{\mathcal{C}_{1}, \mathcal{C}_{1}}^{\mathcal{B}}\right)^{-1}\right]_{j_{1}, j_{1}}+\left[\left(\boldsymbol{J}_{\mathcal{C}_{2}, \mathcal{C}_{2}}^{\mathcal{B}}\right)^{-1}\right]_{j_{2}, j_{2}} \\
\boldsymbol{\Gamma} & =\boldsymbol{K}_{\iota, i_{1}}\left[\left(\boldsymbol{J}_{\mathcal{C}_{1}, \mathcal{C}_{1}}^{\mathcal{B}}\right)^{-1}\right]_{i_{1}, j_{1}}-\boldsymbol{K}_{\iota, i_{2}}\left[\left(\boldsymbol{J}_{\mathcal{C}_{2}, \mathcal{C}_{2}}^{\mathcal{B}}\right)^{-1}\right]_{i_{2}, j_{2}} .
\end{aligned}
$$

Proof: See Appendix E

Note that the coupled information $\Xi_{i_{1}, i_{2}}^{\left(j_{1}, j_{2}\right)}$ in 34 is a function of $\boldsymbol{K}, \boldsymbol{\Phi}$ and $\boldsymbol{\Gamma}$, and we rewrite it as $\boldsymbol{\Xi}_{i_{1}, i_{2}}^{\left(j_{1}, j_{2}\right)}\{\boldsymbol{K}, \boldsymbol{\Phi}, \boldsymbol{\Gamma}\}$ in the following corollary to show the monotonicity of the coupled information with the variables.

Corollary 3: Under the conditions of Theorem 3 ,

1) if $\boldsymbol{K}^{\prime} \succcurlyeq \boldsymbol{K}$

$$
\boldsymbol{\Xi}_{i_{1}, i_{2}}^{\left(j_{1}, j_{2}\right)}\left\{\boldsymbol{K}^{\prime}, \boldsymbol{\Phi}, \boldsymbol{\Gamma}\right\} \succcurlyeq \boldsymbol{\Xi}_{i_{1}, i_{2}}^{\left(j_{1}, j_{2}\right)}\{\boldsymbol{K}, \boldsymbol{\Phi}, \boldsymbol{\Gamma}\} ;
$$

2) if $\boldsymbol{\Phi}^{\prime} \preccurlyeq \Phi$,

$$
\boldsymbol{\Xi}_{i_{1}, i_{2}}^{\left(j_{1}, j_{2}\right)}\left\{\boldsymbol{K}, \boldsymbol{\Phi}^{\prime}, \boldsymbol{\Gamma}\right\} \succcurlyeq \boldsymbol{\Xi}_{i_{1}, i_{2}}^{\left(j_{1}, j_{2}\right)}\{\boldsymbol{K}, \boldsymbol{\Phi}, \boldsymbol{\Gamma}\} ;
$$

3) if $\Gamma^{\prime}=\lambda \Gamma$ with $\lambda \geqslant 1$,

$$
\boldsymbol{\Xi}_{i_{1}, i_{2}}^{\left(j_{1}, j_{2}\right)}\left\{\boldsymbol{K}, \boldsymbol{\Phi}, \boldsymbol{\Gamma}^{\prime}\right\} \succcurlyeq \boldsymbol{\Xi}_{i_{1}, i_{2}}^{\left(j_{1}, j_{2}\right)}\{\boldsymbol{K}, \boldsymbol{\Phi}, \boldsymbol{\Gamma}\} .
$$

Proof: For the first two cases, notice that $\left(\boldsymbol{I}-\boldsymbol{K}_{j_{1}, j_{2}}\left(\boldsymbol{\Phi}^{-1}+\boldsymbol{K}_{j_{1}, j_{2}}\right)^{-1}\right) \boldsymbol{K}_{j_{1}, j_{2}} \quad$ is the Schur complement of the matrix

$$
\boldsymbol{M}=\left[\begin{array}{cc}
\boldsymbol{K}_{j_{1}, j_{2}} & \boldsymbol{K}_{j_{1}, j_{2}} \\
\boldsymbol{K}_{j_{1}, j_{2}} & \boldsymbol{\Phi}^{-\mathbf{1}}+\boldsymbol{K}_{j_{1}, j_{2}}
\end{array}\right]
$$




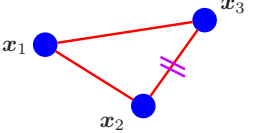

(a)

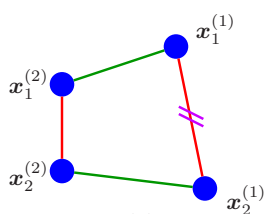

(b)

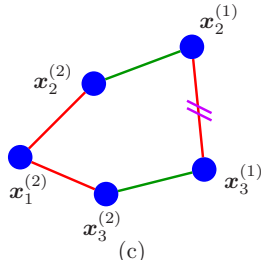

Fig. 2. Three representative simple scenarios of network navigation. (a): Three nodes cooperate via inter-node measurements; (b): Two nodes cooperate at two different times via inter- and intra-node measurements; (c): Three nodes cooperate at two different times via inter- and intra-node measurements. The double slash denotes the interaction set.

Then the results are obtained by observing that $\boldsymbol{K}_{j_{1}, j_{2}}^{\prime} \succcurlyeq$ $\boldsymbol{K}_{j_{1}, j_{2}}$ or $\boldsymbol{\Phi}^{\prime} \preccurlyeq \boldsymbol{\Phi}$ implies that the corresponding matrix $\boldsymbol{M}^{\prime}$ satisfies $M^{\prime} \succcurlyeq \boldsymbol{M}$. The last case is straightforward since $\lambda^{2} \geqslant 1$.

Remark 5: Theorem 3 and Corollary 3 show that the information obtained from cooperation is the sum of effective information from each neighbor plus the coupled information by the link in the interaction set. Such coupled information depends on the link capacities between state $\iota$ and the coupled neighbors, as well as on the link capacity of the interaction set. In addition, the coupled information increases when 1) the capacity of the link in the interaction set increases, 2) the uncertainty of the interaction set decreases, and 3) the capacities of the links to the coupled neighbors increase proportionally with the same ratio.

Corollary 4: Under the conditions of Theorem 3 , if $\boldsymbol{K}_{\iota, i_{1}}=$

$$
\begin{gathered}
\boldsymbol{v}_{\iota, i_{1}} \boldsymbol{v}_{\iota, i_{1}}^{\mathrm{T}}, \boldsymbol{K}_{\iota, i_{2}}=\boldsymbol{v}_{\iota, i_{2}} \boldsymbol{v}_{\iota, i_{2}}^{\mathrm{T}} \text {, and } \boldsymbol{K}_{j_{1}, j_{2}}=\boldsymbol{v}_{j_{1}, j_{2}} \boldsymbol{v}_{j_{1}, j_{2}}^{\mathrm{T}} \text {, then } \\
\boldsymbol{\Xi}_{i_{1}, i_{2}}^{\left(j_{1}, j_{2}\right)}=\delta_{j_{1}, j_{2}}\left(\mu_{i_{1}} \boldsymbol{v}_{\iota, i_{1}}-\mu_{i_{2}} \boldsymbol{v}_{\iota, i_{2}}\right)\left(\mu_{i_{1}} \boldsymbol{v}_{\iota, i_{1}}-\mu_{i_{2}} \boldsymbol{v}_{\iota, i_{2}}\right)^{\mathrm{T}}
\end{gathered}
$$

where

$$
\begin{aligned}
\delta_{j_{1}, j_{2}} & =\left(1+\boldsymbol{v}_{j_{1}, j_{2}}^{\mathrm{T}} \boldsymbol{\Phi} \boldsymbol{v}_{j_{1}, j_{2}}\right)^{-1} \\
\mu_{i_{1}} & =\boldsymbol{v}_{\iota, i_{1}}^{\mathrm{T}}\left[\left(\boldsymbol{J}_{\mathcal{C}_{1}, \mathcal{C}_{1}}^{\mathcal{B}}\right)^{-1}\right]_{i_{1}, j_{1}} \boldsymbol{v}_{j_{1}, j_{2}} \\
\mu_{i_{2}} & =\boldsymbol{v}_{\iota, i_{2}}^{\mathrm{T}}\left[\left(\boldsymbol{J}_{\mathcal{C}_{2}, \mathcal{C}_{2}}^{\mathcal{B}}\right)^{-1}\right]_{i_{2}, j_{2}} \boldsymbol{v}_{j_{1}, j_{2}} .
\end{aligned}
$$

Proof: See Appendix F

Remark 6: This result shows that when the information capacities of the coupled neighbors and interaction set are rank-one matrices, the coupled information is also a rankone matrix. In addition, such matrix is the outer product of a combination of the vectors forming the link capacity from the neighbors weighted by the coefficients $\mu_{i_{1}} / \sqrt{\delta_{j_{1}, j_{2}}}$ and $-\mu_{i_{2}} / \sqrt{\delta_{j_{1}, j_{2}}}$.

The next section focuses on four representative cases and derives the corresponding EFIMs. In Section $\nabla$ these scenarios are further examined and the coupled information is numerically quantified.

\section{CASE STUdiES}

This section presents the EFIMs for a state in the scenarios depicted in Fig. 2 and Fig. 3 to provide insights into the spatiotemporal information coupling arising in network navigation. These scenarios are composed by small-scale networks with 3,4 , and 5 states as well as by a simple network with $2 n+1$ states.

In the following, the link capacities $\boldsymbol{K}_{i, j}$ for $i \neq j$ account for the positional information obtained from the inter- or intra-node measurements. When inter-node measurements are related to the distances between pairs of nodes and intra-node measurements are related to the speed of nodes, each $\boldsymbol{K}_{i, j}$ for $i \neq j$ is a rank-one matrix. Specifically, $\boldsymbol{K}_{i, j}=\boldsymbol{v}_{i, j} \boldsymbol{v}_{i, j}^{\mathrm{T}}$, and $\boldsymbol{v}_{i, j}$ is a vector with direction joining states $i$ and $j$ and norm $\sqrt{\lambda_{i, j}} \in \mathbb{R}_{+}$, where $\lambda_{i, j}$ is called the ranging information intensity (RII) [32] and speed information intensity (SII) [71] for inter- and intra-node measurements, respectively.

\section{A. Scenario A}

Three nodes cooperate to determine their positions via internode measurements, e.g., time-of-arrival (TOA) or angle-ofarrival (AOA), as described in Fig. 2(a). In this case, the states are $\boldsymbol{x}_{1}, \boldsymbol{x}_{2}$, and $\boldsymbol{x}_{3}$ (denoted by states 1,2 , and 3 , respectively), which represent the positions of the three nodes, and the FIM becomes 7

$$
\boldsymbol{J}=\left[\begin{array}{ccc}
\boldsymbol{J}_{\{1\},\{1\}} & -\boldsymbol{K}_{1,2} & -\boldsymbol{K}_{1,3} \\
-\boldsymbol{K}_{1,2} & \boldsymbol{J}_{\{2\},\{2\}} & -\boldsymbol{K}_{2,3} \\
-\boldsymbol{K}_{1,3} & -\boldsymbol{K}_{2,3} & \boldsymbol{J}_{\{3\},\{3\}}
\end{array}\right]
$$

where

$$
\begin{aligned}
& \boldsymbol{J}_{\{1\},\{1\}}=\boldsymbol{K}_{1,1}+\boldsymbol{K}_{1,2}+\boldsymbol{K}_{1,3} \\
& \boldsymbol{J}_{\{2\},\{2\}}=\boldsymbol{K}_{2,2}+\boldsymbol{K}_{1,2}+\boldsymbol{K}_{2,3} \\
& \boldsymbol{J}_{\{3\},\{3\}}=\boldsymbol{K}_{3,3}+\boldsymbol{K}_{1,3}+\boldsymbol{K}_{2,3} .
\end{aligned}
$$

As a special case of Theorem 3 with interaction set $\mathcal{B}=$ $\{(2,3)\}$, the EFIM for $\boldsymbol{x}_{1}$ in Scenario A is (see Appendix G)

$$
\boldsymbol{J}_{\mathrm{e}}=\boldsymbol{K}_{1,1}+\boldsymbol{K}_{2 \rightarrow 1}^{(2,3)}+\boldsymbol{K}_{3 \rightarrow 1}^{(2,3)}+\boldsymbol{\Xi}_{2,3}^{(2,3)}
$$

where

$$
\begin{aligned}
\boldsymbol{\Xi}_{2,3}^{(2,3)} & =\boldsymbol{\Gamma}\left(\boldsymbol{K}_{2,3}\left(\boldsymbol{\Phi}^{-1}+\boldsymbol{K}_{2,3}\right)^{-1} \boldsymbol{\Phi}^{-1}\right) \boldsymbol{\Gamma}^{\mathrm{T}} \\
\boldsymbol{\Gamma} & =\boldsymbol{K}_{1,2}\left(\boldsymbol{K}_{2,2}+\boldsymbol{K}_{1,2}\right)^{-1}-\boldsymbol{K}_{1,3}\left(\boldsymbol{K}_{3,3}+\boldsymbol{K}_{1,3}\right)^{-1} \\
\boldsymbol{\Phi} & =\left(\boldsymbol{K}_{2,2}+\boldsymbol{K}_{1,2}\right)^{-1}+\left(\boldsymbol{K}_{3,3}+\boldsymbol{K}_{1,3}\right)^{-1}
\end{aligned}
$$

and

$$
\begin{aligned}
\boldsymbol{K}_{i \rightarrow 1}^{(2,3)} & =\left(\boldsymbol{I}-\boldsymbol{K}_{1, i}\left(\boldsymbol{K}_{i, i}+\boldsymbol{K}_{1, i}\right)^{-1}\right) \boldsymbol{K}_{1, i} \\
& =\boldsymbol{K}_{1, i}\left(\boldsymbol{K}_{i, i}+\boldsymbol{K}_{1, i}\right)^{-1} \boldsymbol{K}_{i, i} .
\end{aligned}
$$

The EFIM in (43) is the sum of four terms. The first term corresponds to the information that depends only on node 1 , and the remaining three terms correspond to the information obtained through spatial cooperation. The second and third terms correspond to the decoupled effective information obtained from neighbor nodes 2 and 3, respectively, while the last term represents the coupled information due to the cooperation between neighbor nodes 2 and 3. The coupled information depends on the capacity of the link between nodes 2 and 3 as well as the uncertainties of nodes 2 and 3 .

\footnotetext{
${ }^{7}$ Notice that in this scenario for notational convenience we do not specify the time index because there is only one time step involved.
} 
If the link capacities are rank-one matrices, i.e., $\boldsymbol{K}_{i, j}=$ $\boldsymbol{v}_{i, j} \boldsymbol{v}_{i, j}^{\mathrm{T}}$ with $\boldsymbol{v}_{i, j} \in \mathbb{R}^{D}$ for $i, j \in\{1,2,3\}, i \neq j$, then the EFIM $J_{\mathrm{e}}$ can be simplified as (see Appendix $\mathrm{G}$ )

$$
\begin{aligned}
\boldsymbol{J}_{\mathrm{e}}= & \boldsymbol{K}_{1,1}+\xi_{1,2} \boldsymbol{K}_{1,2}+\xi_{1,3} \boldsymbol{K}_{1,3} \\
& +\delta_{2,3}\left(\mu_{2} \boldsymbol{v}_{1,2}-\mu_{3} \boldsymbol{v}_{1,3}\right)\left(\mu_{2} \boldsymbol{v}_{1,2}-\mu_{3} \boldsymbol{v}_{1,3}\right)^{\mathrm{T}}
\end{aligned}
$$

where

$$
\begin{aligned}
\xi_{1, i} & =\left(1+\boldsymbol{v}_{1, i}^{\mathrm{T}} \boldsymbol{K}_{i, i}^{-1} \boldsymbol{v}_{1, i}\right)^{-1} \\
\mu_{i} & =\xi_{1, i}\left(\boldsymbol{v}_{1, i}^{\mathrm{T}} \boldsymbol{K}_{i, i}^{-1} \boldsymbol{v}_{2,3}\right) \\
\delta_{2,3} & =
\end{aligned}
$$

$$
\left(1+\boldsymbol{v}_{2,3}^{\mathrm{T}}\left(\left(\boldsymbol{K}_{2,2}+\boldsymbol{K}_{1,2}\right)^{-1}+\left(\boldsymbol{K}_{3,3}+\boldsymbol{K}_{1,3}\right)^{-1}\right) \boldsymbol{v}_{2,3}\right)^{-1} .
$$

In this special case, each effective link information for node 1 is proportional to the corresponding link capacity. The coupled information is also a rank-one matrix with positive eigenvalue $\delta_{2,3}\left\|\mu_{2} \boldsymbol{v}_{1,2}-\mu_{3} \boldsymbol{v}_{1,3}\right\|^{2}$ and corresponding eigenvector $\mu_{2} \boldsymbol{v}_{1,2}-\mu_{3} \boldsymbol{v}_{1,3}$, both depending on the RIIs, the uncertainties of nodes 2 and 3 , and the nodes' spatial topology.

\section{B. Scenario $B$}

Two nodes cooperate at two consecutive time steps to determine their positions via inter- and intra-node measurements, as described in Fig. 2(b). In this case, the states are $\boldsymbol{x}_{1}^{(2)}, \boldsymbol{x}_{2}^{(2)}$, $\boldsymbol{x}_{1}^{(1)}$, and $\boldsymbol{x}_{2}^{(1)}$ (denoted by states $1,2,3$, and 4 , respectively), which represent the states of the two nodes at two time steps. Note that $\boldsymbol{K}_{1,4}=\boldsymbol{K}_{2,3}=0$, and hence the FIM becomes

$$
\boldsymbol{J}=\left[\begin{array}{cccc}
\boldsymbol{J}_{\{1\},\{1\}} & -\boldsymbol{K}_{1,2} & -\boldsymbol{K}_{1,3} & 0 \\
-\boldsymbol{K}_{1,2} & \boldsymbol{J}_{\{2\},\{2\}} & 0 & -\boldsymbol{K}_{2,4} \\
-\boldsymbol{K}_{1,3} & 0 & \boldsymbol{J}_{\{3\},\{3\}} & -\boldsymbol{K}_{3,4} \\
0 & -\boldsymbol{K}_{2,4} & -\boldsymbol{K}_{3,4} & \boldsymbol{J}_{\{4\},\{4\}}
\end{array}\right]
$$

where

$$
\begin{aligned}
& \boldsymbol{J}_{\{1\},\{1\}}=\boldsymbol{K}_{1,1}+\boldsymbol{K}_{1,2}+\boldsymbol{K}_{1,3} \\
& \boldsymbol{J}_{\{2\},\{2\}}=\boldsymbol{K}_{2,2}+\boldsymbol{K}_{1,2}+\boldsymbol{K}_{2,4} \\
& \boldsymbol{J}_{\{3\},\{3\}}=\boldsymbol{K}_{3,3}+\boldsymbol{K}_{1,3}+\boldsymbol{K}_{3,4} \\
& \boldsymbol{J}_{\{4\},\{4\}}=\boldsymbol{K}_{4,4}+\boldsymbol{K}_{2,4}+\boldsymbol{K}_{3,4} .
\end{aligned}
$$

As a special case of Theorem 3 with interaction set $\mathcal{B}=$ $\{(4,3)\}$ the EFIM for $\boldsymbol{x}_{1}^{(2)}$ in Scenario B is (see Appendix G

$$
\boldsymbol{J}_{\mathrm{e}}=\boldsymbol{K}_{1,1}+\boldsymbol{K}_{2 \rightarrow 1}^{(4,3)}+\boldsymbol{K}_{3 \rightarrow 1}^{(4,3)}+\boldsymbol{\Xi}_{2,3}^{(4,3)}
$$

where

$$
\begin{aligned}
\boldsymbol{\Xi}_{2,3}^{(4,3)}= & \boldsymbol{\Gamma}\left(\boldsymbol{K}_{4,3}\left(\boldsymbol{\Phi}^{-1}+\boldsymbol{K}_{4,3}\right)^{-1} \boldsymbol{\Phi}^{-1}\right) \boldsymbol{\Gamma}^{\mathrm{T}} \\
\boldsymbol{\Gamma}= & \boldsymbol{K}_{1,2} \boldsymbol{\Omega}_{2}^{-1} \boldsymbol{K}_{2,4}\left(\boldsymbol{K}_{4,4}+\boldsymbol{K}_{2,4}\right)^{-1} \\
& \quad-\boldsymbol{K}_{1,3}\left(\boldsymbol{K}_{3,3}+\boldsymbol{K}_{1,3}\right)^{-1} \\
\boldsymbol{\Phi}= & \boldsymbol{\Omega}_{4}^{-1}+\left(\boldsymbol{K}_{3,3}+\boldsymbol{K}_{1,3}\right)^{-1} \\
\boldsymbol{K}_{2 \rightarrow 1}^{(4,3)}= & \left(\boldsymbol{I}-\boldsymbol{K}_{1,2} \boldsymbol{\Omega}_{2}^{-1}\right) \boldsymbol{K}_{1,2} \\
\boldsymbol{K}_{3 \rightarrow 1}^{(4,3)}= & \left(\boldsymbol{I}-\boldsymbol{K}_{1,3}\left(\boldsymbol{K}_{3,3}+\boldsymbol{K}_{1,3}\right)^{-1}\right) \boldsymbol{K}_{1,3}
\end{aligned}
$$

${ }^{8}$ Note that one can also consider the interaction set to be $\mathcal{B}=\{(4,2)\}$ which leads to analogous results. and

$$
\begin{aligned}
& \boldsymbol{\Omega}_{2}=\boldsymbol{K}_{2,2}+\boldsymbol{K}_{1,2}+\left(\boldsymbol{I}-\boldsymbol{K}_{2,4}\left(\boldsymbol{K}_{4,4}+\boldsymbol{K}_{2,4}\right)^{-1}\right) \boldsymbol{K}_{2,4} \\
& \boldsymbol{\Omega}_{4}=\boldsymbol{K}_{4,4}+\left(\boldsymbol{I}-\boldsymbol{K}_{2,4}\left(\boldsymbol{K}_{2,2}+\boldsymbol{K}_{1,2}+\boldsymbol{K}_{2,4}\right)^{-1}\right) \boldsymbol{K}_{2,4} .
\end{aligned}
$$

The EFIM in (50) is the sum of four terms. The first term corresponds to the information that depends only on node 1 at time step $t_{2}$, and the remaining three terms correspond to the information obtained through spatiotemporal cooperation. The second and third terms correspond to the effective information obtained from spatial neighbor (node 2 at time step $t_{2}$ ) and temporal neighbor (node 1 at time step $t_{1}$ ), respectively, while the last term represents the coupled information due to the cooperation between neighbors (nodes 1 and 2 share information at time step $t_{1}$ ). The coupled information depends on the capacity of the link between nodes 1 and 2 at time step $t_{1}$ as well as the uncertainties of node 2 at time $t_{2}$ and node 1 at time $t_{1}$.

If the link capacities are all rank-one matrices, i.e., $\boldsymbol{K}_{1,2}=$ $\boldsymbol{v}_{1,2} \boldsymbol{v}_{1,2}^{\mathrm{T}}, \boldsymbol{K}_{1,3}=\boldsymbol{v}_{1,3} \boldsymbol{v}_{1,3}^{\mathrm{T}}$, and $\boldsymbol{K}_{3,4}=\boldsymbol{v}_{3,4} \boldsymbol{v}_{3,4}^{\mathrm{T}}$, then the EFIM $\boldsymbol{J}_{\mathrm{e}}$ can be simplified as (see Appendix G)

$$
\begin{aligned}
\boldsymbol{J}_{\mathrm{e}}= & \boldsymbol{K}_{1,1}+\xi_{1,2} \boldsymbol{K}_{1,2}+\xi_{1,3} \boldsymbol{K}_{1,3} \\
& +\delta_{3,4}\left(\mu_{2} \boldsymbol{v}_{1,2}-\mu_{3} \boldsymbol{v}_{1,3}\right)\left(\mu_{2} \boldsymbol{v}_{1,2}-\mu_{3} \boldsymbol{v}_{1,3}\right)^{\mathrm{T}}
\end{aligned}
$$

where

$$
\begin{aligned}
& \xi_{1,2}= \\
&\left(1+\boldsymbol{v}_{1,2}^{\mathrm{T}}\left(\boldsymbol{K}_{2,2}+\left(1+\boldsymbol{v}_{2,4}^{\mathrm{T}} \boldsymbol{K}_{4,4}^{-1} \boldsymbol{v}_{2,4}\right)^{-1} \boldsymbol{K}_{2,4}\right)^{-1} \boldsymbol{v}_{1,2}\right)^{-1} \\
& \xi_{1,3}=\left(1+\boldsymbol{v}_{1,3}^{\mathrm{T}} \boldsymbol{K}_{3,3}^{-1} \boldsymbol{v}_{1,3}\right)^{-1} \\
& \mu_{2}=\xi_{1,2}\left(1+\boldsymbol{v}_{2,4}^{\mathrm{T}} \boldsymbol{K}_{4,4}^{-1} \boldsymbol{v}_{2,4}\right)^{-1}\left(\boldsymbol{v}_{2,4}^{\mathrm{T}} \boldsymbol{K}_{4,4}^{-1} \boldsymbol{v}_{3,4}\right) \\
& \cdot \boldsymbol{v}_{1,2}^{\mathrm{T}}\left(\boldsymbol{K}_{2,2}+\left(1+\boldsymbol{v}_{2,4}^{\mathrm{T}} \boldsymbol{K}_{4,4}^{-1} \boldsymbol{v}_{2,4}\right)^{-1} \boldsymbol{K}_{2,4}\right)^{-1} \boldsymbol{v}_{2,4} \\
& \mu_{3}= \xi_{1,3}\left(\boldsymbol{v}_{1,3}^{\mathrm{T}} \boldsymbol{K}_{3,3}^{-1} \boldsymbol{v}_{3,4}\right) \\
& \delta_{3,4}=\left(1+\boldsymbol{v}_{3,4}^{\mathrm{T}}\left(\boldsymbol{\Omega}_{4}^{-1}+\left(\boldsymbol{K}_{3,3}+\boldsymbol{K}_{1,3}\right)^{-1}\right) \boldsymbol{v}_{3,4}\right)^{-1} .
\end{aligned}
$$

In this special case, each effective link information for node 1 is proportional to the corresponding link capacity. The coupled information is a rank-one matrix with positive eigenvalue $\delta_{3,4}\left\|\mu_{2} \boldsymbol{v}_{1,2}-\mu_{3} \boldsymbol{v}_{1,3}\right\|^{2}$ and corresponding eigenvector $\mu_{2} \boldsymbol{v}_{1,2}-\mu_{3} \boldsymbol{v}_{1,3}$ both depending on the RIIs, the SIIs, the uncertainties of nodes 1 and 2 at different time steps, and the nodes' spatial topology.

\section{Scenario $C$}

Node 1 cooperates with nodes 2 and 3 at time step $t_{2}$ while nodes 2 and 3 have cooperated at time step $t_{1}$, as described in Fig. 2( c). In this case, the states are $\boldsymbol{x}_{1}^{(2)}, \boldsymbol{x}_{2}^{(2)}, \boldsymbol{x}_{3}^{(2)}, \boldsymbol{x}_{2}^{(1)}$, and $\boldsymbol{x}_{3}^{(1)}$ (denoted by states $1,2,3,4$, and 5 , respectively), which represent the states of three nodes at time step $t_{2}$ and two nodes at time step $t_{1}$. Then, the FIM becomes

$$
\boldsymbol{J}=\left[\begin{array}{ccccc}
\boldsymbol{J}_{\{1\},\{1\}} & -\boldsymbol{K}_{1,2} & -\boldsymbol{K}_{1,3} & 0 & 0 \\
-\boldsymbol{K}_{1,2} & \boldsymbol{J}_{\{2\},\{2\}} & 0 & -\boldsymbol{K}_{2,4} & 0 \\
-\boldsymbol{K}_{1,3} & 0 & \boldsymbol{J}_{\{3\},\{3\}} & 0 & -\boldsymbol{K}_{3,5} \\
0 & -\boldsymbol{K}_{2,4} & 0 & \boldsymbol{J}_{\{4\},\{4\}} & -\boldsymbol{K}_{4,5} \\
0 & 0 & -\boldsymbol{K}_{3,5} & -\boldsymbol{K}_{4,5} & \boldsymbol{J}_{\{5\},\{5\}}
\end{array}\right]
$$


where

$$
\begin{aligned}
& \boldsymbol{J}_{\{1\},\{1\}}=\boldsymbol{K}_{1,1}+\boldsymbol{K}_{1,2}+\boldsymbol{K}_{1,3} \\
& \boldsymbol{J}_{\{2\},\{2\}}=\boldsymbol{K}_{2,2}+\boldsymbol{K}_{1,2}+\boldsymbol{K}_{2,4} \\
& \boldsymbol{J}_{\{3\},\{3\}}=\boldsymbol{K}_{3,3}+\boldsymbol{K}_{1,3}+\boldsymbol{K}_{3,5} \\
& \boldsymbol{J}_{\{4\},\{4\}}=\boldsymbol{K}_{4,4}+\boldsymbol{K}_{2,4}+\boldsymbol{K}_{4,5} \\
& \boldsymbol{J}_{\{5\},\{5\}}=\boldsymbol{K}_{5,5}+\boldsymbol{K}_{3,5}+\boldsymbol{K}_{4,5} .
\end{aligned}
$$

As a special case of Theorem 3 with interaction set $\mathcal{B}=$ $\{(4,5)\}$, 9 the EFIM for $\boldsymbol{x}_{1}^{(2)}$ in Scenario C is (see Appendix G]

$$
\boldsymbol{J}_{\mathrm{e}}=\boldsymbol{K}_{1,1}+\boldsymbol{K}_{2 \rightarrow 1}^{(4,5)}+\boldsymbol{K}_{3 \rightarrow 1}^{(4,5)}+\boldsymbol{\Xi}_{2,3}^{(4,5)}
$$

where

$$
\begin{aligned}
\boldsymbol{\Xi}_{2,3}^{(4,5)}= & \boldsymbol{\Gamma}\left(\boldsymbol{I}-\boldsymbol{K}_{4,5}\left(\boldsymbol{\Phi}^{-1}+\boldsymbol{K}_{4,5}\right)^{-1}\right) \boldsymbol{K}_{4,5} \boldsymbol{\Gamma}^{\mathrm{T}} \\
\boldsymbol{\Gamma}= & \boldsymbol{K}_{1,2} \boldsymbol{\Omega}_{2}^{-1} \boldsymbol{K}_{2,4}\left(\boldsymbol{K}_{4,4}+\boldsymbol{K}_{2,4}\right)^{-1} \\
& -\boldsymbol{K}_{1,3} \boldsymbol{\Omega}_{3}^{-1} \boldsymbol{K}_{3,5}\left(\boldsymbol{K}_{5,5}+\boldsymbol{K}_{3,5}\right)^{-1} \\
\boldsymbol{\Phi}= & \boldsymbol{\Omega}_{4}^{-1}+\boldsymbol{\Omega}_{5}^{-1} \\
\boldsymbol{K}_{2 \rightarrow 1}^{(4,5)}= & \left(\boldsymbol{I}-\boldsymbol{K}_{1,2} \boldsymbol{\Omega}_{2}^{-1}\right) \boldsymbol{K}_{1,2} \\
\boldsymbol{K}_{3 \rightarrow 1}^{(4,5)}= & \left(\boldsymbol{I}-\boldsymbol{K}_{1,3} \boldsymbol{\Omega}_{3}^{-1}\right) \boldsymbol{K}_{1,3} \\
\boldsymbol{\Omega}_{i}= & \boldsymbol{K}_{i, i}+\boldsymbol{K}_{1, i} \\
& +\left(\boldsymbol{I}-\boldsymbol{K}_{i, i+2}\left(\boldsymbol{K}_{i+2, i+2}+\boldsymbol{K}_{i, i+2}\right)^{-1}\right) \boldsymbol{K}_{i, i+2}
\end{aligned}
$$

for $i=2,3$, and

$$
\begin{aligned}
\boldsymbol{\Omega}_{i} & =\boldsymbol{K}_{i, i} \\
& +\left(\boldsymbol{I}-\boldsymbol{K}_{i-2, i}\left(\boldsymbol{K}_{i-2, i-2}+\boldsymbol{K}_{1, i-2}+\boldsymbol{K}_{i-2, i}\right)^{-1}\right) \boldsymbol{K}_{i-2, i}
\end{aligned}
$$

for $i=4,5$.

The EFIM in 57 is the sum of four terms. The first term corresponds to the information that depends only on node 1 at time step $t_{2}$, and the remaining 3 terms correspond to the information obtained through spatiotemporal cooperation. The second and third terms correspond to the decoupled effective information obtained from spatial neighbors (nodes 2 and 3 at time step $t_{2}$ ), respectively, while the last term represents the coupled information due to the cooperation between neighbors (nodes 2 and 3 share information at time step $t_{1}$ ). The coupled information depends on the capacity of the link between nodes 2 and 3 at time step $t_{1}$ as well as the uncertainties of nodes 2 and 3 at time $t_{2}$.

If the link capacities are all rank-one matrices, i.e., $\boldsymbol{K}_{1, i}=$ $\boldsymbol{v}_{1, i} \boldsymbol{v}_{1, i}^{\mathrm{T}}, \boldsymbol{K}_{i, i+2}=\boldsymbol{v}_{i, i+2} \boldsymbol{v}_{i, i+2}^{\mathrm{T}}$ for $i=2,3$, and $\boldsymbol{K}_{4,5}=$ $\boldsymbol{v}_{4,5} \boldsymbol{v}_{4,5}^{\mathrm{T}}$, then the EFIM $\boldsymbol{J}_{\mathrm{e}}$ can be simplified as (see Appendix G]

$$
\begin{aligned}
\boldsymbol{J}_{\mathrm{e}}= & \boldsymbol{K}_{1,1}+\xi_{1,2} \boldsymbol{K}_{1,2}+\xi_{1,3} \boldsymbol{K}_{1,3} \\
& +\delta_{4,5}\left(\mu_{2} \boldsymbol{v}_{1,2}-\mu_{3} \boldsymbol{v}_{1,3}\right)\left(\mu_{2} \boldsymbol{v}_{1,2}-\mu_{3} \boldsymbol{v}_{1,3}\right)^{\mathrm{T}}
\end{aligned}
$$

where

$$
\begin{aligned}
& \xi_{1, i}= \\
& \frac{1}{1+\boldsymbol{v}_{1, i}^{\mathrm{T}}\left(\boldsymbol{K}_{i, i}+\left(1+\boldsymbol{v}_{i, i+2}^{\mathrm{T}} \boldsymbol{K}_{i+2, i+2}^{-1} \boldsymbol{v}_{i, i+2}\right)^{-1} \boldsymbol{K}_{i, i+2}\right)^{-1} \boldsymbol{v}_{1, i}}
\end{aligned}
$$

${ }^{9}$ Note that one can also consider the interaction set to be $\mathcal{B}=\{(4,2)\}$ and $\mathcal{B}=\{(3,5)\}$, which lead to analogous results.

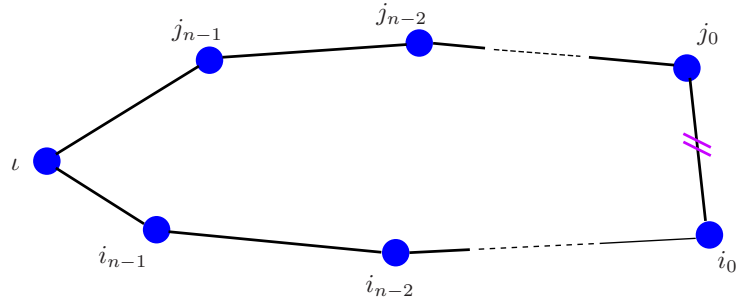

Fig. 3. A simple scenario of network navigation in which all the nodes are linked by a single loop. The double slash denotes a potential interaction set.

$$
\begin{aligned}
\mu_{i}= & \xi_{1, i}\left(1+\boldsymbol{v}_{i, i+2}^{\mathrm{T}} \boldsymbol{K}_{i+2, i+2}^{-1} \boldsymbol{v}_{i, i+2}\right)^{-1}\left(\boldsymbol{v}_{i, i+2}^{\mathrm{T}} \boldsymbol{K}_{i+2, i+2}^{-1} \boldsymbol{v}_{4,5}\right) \\
\cdot & \boldsymbol{v}_{1, i}^{\mathrm{T}}\left(\boldsymbol{K}_{i, i}+\left(1+\boldsymbol{v}_{i, i+2}^{\mathrm{T}} \boldsymbol{K}_{i+2, i+2}^{-1} \boldsymbol{v}_{i, i+2}\right)^{-1} \boldsymbol{K}_{i, i+2}\right)^{-1} \boldsymbol{v}_{i, i+2}
\end{aligned}
$$

for $i=2,3$, and

$$
\delta_{4,5}=\left(1+\boldsymbol{v}_{4,5}^{\mathrm{T}}\left(\boldsymbol{\Omega}_{4}^{-1}+\boldsymbol{\Omega}_{5}^{-1}\right) \boldsymbol{v}_{4,5}\right)^{-1} .
$$

In this case, each effective link information for node 1 is proportional to the corresponding link capacity. The coupled information is a rank-one matrix with positive eigenvalue $\delta_{4,5}\left\|\mu_{2} \boldsymbol{v}_{1,2}-\mu_{3} \boldsymbol{v}_{1,3}\right\|^{2}$ and corresponding eigenvector $\mu_{2} \boldsymbol{v}_{1,2}-\mu_{3} \boldsymbol{v}_{1,3}$ both depending on the RIIs, the SIIs, the uncertainties of nodes 1,2 and 3 at different time steps, and the nodes' spatial topology.

\section{Scenario D}

Finally, we characterize the exponential decay of information coupling with respect to the network distance for the simple network shown in Fig. 3. This behavior is also observed in Section $\mathrm{V}$ for more general networks.

In Fig. 3, the subset of the states with network distance at least $k$ to $\mathcal{B}=\left\{i_{0}, j_{0}\right\}$ is

$$
\mathcal{S}_{k}=\{\iota\} \cup\left\{\left(i_{r}, j_{r}\right)\right\}_{r \geqslant k}, \quad k=0,1, \ldots, n
$$

and the subset of the states with network distance $k$ to $\mathcal{B}=$ $\left\{\left(i_{0}, j_{0}\right)\right\}$ is

$$
\mathcal{E}_{k}= \begin{cases}\left\{\left(i_{k}, j_{k}\right)\right\}, & k=0,1, \ldots, n-1 \\ \{\iota\}, & k=n .\end{cases}
$$

Then, as a consequence of Theorem 2 and Corollary 2 we have the following result.

Corollary 5: Let $\mathcal{S}$ be a set of the states with NIG described by Fig. 31, (64), and (65), with $\boldsymbol{K}_{s, r}=\boldsymbol{K}_{r, s}=\lambda_{r, s} \boldsymbol{I}$ for $r, s \in\left\{i_{0}, i_{1}, \ldots, i_{n-1}, j_{0}, j_{1}, \ldots, j_{n-1}\right\}$. Then, the coupled information for state $\iota$ induced by $\mathcal{B}=\left\{\left(i_{0}, j_{0}\right)\right\}$ is upper bounded as

$$
\boldsymbol{\Xi}^{\left(i_{0}, j_{0}\right)} \preccurlyeq 4 \lambda_{i_{0}, j_{0}} \varepsilon^{n} \boldsymbol{I}
$$

where $\varepsilon=\left(\lambda_{2} /\left(\lambda_{1}+\lambda_{2}\right)\right)^{2}<1, \lambda_{1}=\min \left\{\lambda_{r, r}\right\}$, and $\lambda_{2}=$ $\max \left\{\lambda_{r, s}\right\}$ for $r, s \in\left\{i_{0}, i_{1}, \ldots, i_{n-1}, j_{0}, j_{1}, \ldots, j_{n-1}\right\}$.

Proof: See Appendix $\mathrm{H}$

Remark 7: This result shows that when two neighbors are linked by a single loop $\left\{i_{n-1}, i_{n-2}, \ldots, i_{0}, j_{0}, j_{1}, \ldots, j_{n-1}\right\}$ 


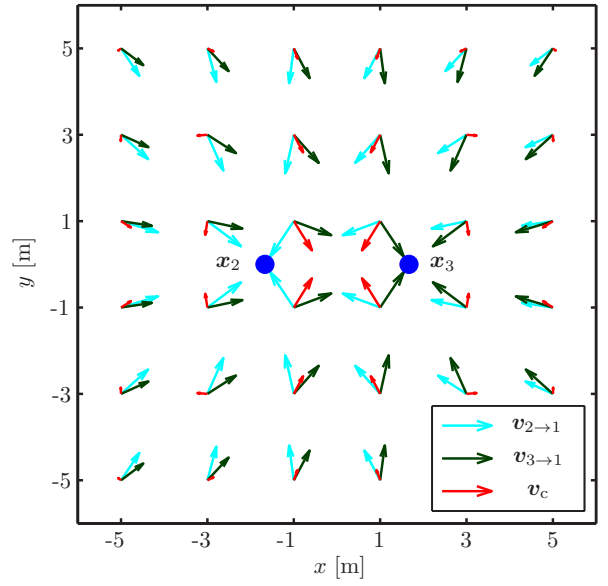

(a)

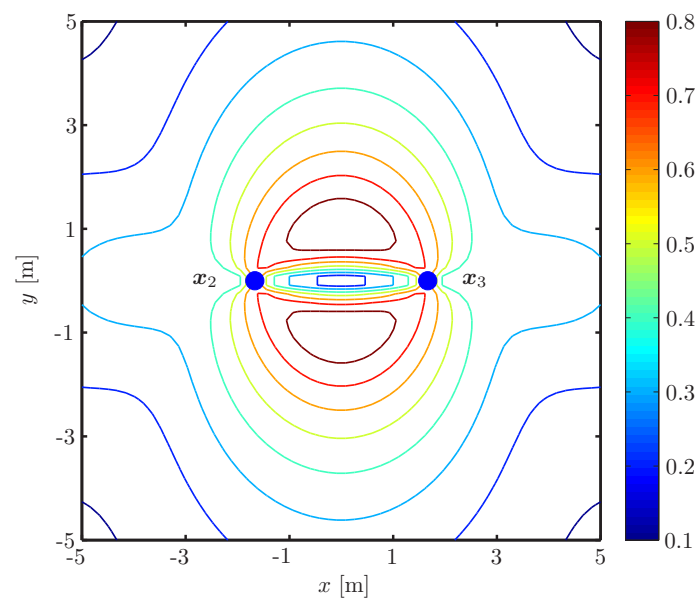

(b)

Fig. 4. Quantification of the coupled information for Scenario A. (a): Vectors $\boldsymbol{v}_{2 \rightarrow 1}, \boldsymbol{v}_{3 \rightarrow 1}$, and $\boldsymbol{v}_{\mathrm{c}}$ representing the effective information and the coupled information from cooperation with nodes 2 and 3; (b): Intensity of information coupling for different values $\boldsymbol{x}_{1}$, measured by the norm of vector $\boldsymbol{v}_{\mathrm{c}}$ (nuclear norm of $\Xi_{2,3}^{(2,3)}$.

and the link capacities are "isotropic," the information coupling induced by the interaction set $\left\{i_{0}, j_{0}\right\}$ decays exponentially with the network distance of the interaction set. This exponential decay as a function of the distance of the links forming the interaction set is further examined in Section $\nabla$ for more general networks through numerical results.

The exponential decay of coupled information implies that only close links induce a significant information coupling. This locality of the information coupling can be exploited by distributed algorithms since the effect of information coupling can be safely discarded when the neighbors are coupled by distant links. Hence, distributed algorithms that track the information coupling induced only by close links can result in near-optimal implementations.

\section{NumericAl RESUltS}

This section presents numerical results quantifying the coupled information. We first assess the effect of information coupling for scenarios $\mathrm{A}, \mathrm{B}$, and $\mathrm{C}$ studied in the previous section, then we describe the effect of information coupling for large networks and how it decreases with the network distance of the links inducing the coupling.

\section{A. Representative Scenarios}

We simulate the three specific scenarios in the square $[-5 \mathrm{~m}, 5 \mathrm{~m}] \times[-5 \mathrm{~m}, 5 \mathrm{~m}]$ and quantify the terms corresponding to the effective information from neighbors as well as the coupled information.

1) Scenario A: In this scenario, the positions of nodes 2 and 3 are $(-1.66 \mathrm{~m}, 0 \mathrm{~m})$ and $(1.66 \mathrm{~m}, 0 \mathrm{~m})$, the matrices $\boldsymbol{K}_{i, i}=$ $\boldsymbol{I}$, for $i=1,2,3$, and the RIIs $\lambda_{i, j}=50 / d_{i, j}^{2}$ for $i \neq j$ [32] 10 Fig. 4(a) shows the vectors $\boldsymbol{v}_{2 \rightarrow 1}, \boldsymbol{v}_{3 \rightarrow 1}$, and $\boldsymbol{v}_{\mathrm{c}}$ where

\footnotetext{
${ }^{10}$ For simplicity, we illustrate the numerical results of the information coupling using the free-space path-loss model for the wireless ranging signals. Similar observations can be made with other distributions for fading models.
}

$\boldsymbol{K}_{2 \rightarrow 1}^{(2,3)}=\boldsymbol{v}_{2 \rightarrow 1} \boldsymbol{v}_{2 \rightarrow 1}^{\mathrm{T}}, \boldsymbol{K}_{3 \rightarrow 1}^{(2,3)}=\boldsymbol{v}_{3 \rightarrow 1} \boldsymbol{v}_{3 \rightarrow 1}^{\mathrm{T}}$, and $\boldsymbol{\Xi}_{2,3}^{(2,3)}=$ $\boldsymbol{v}_{\mathrm{c}} \boldsymbol{v}_{\mathrm{c}}^{\mathrm{T}}$ for a grid of 36 different values of $\boldsymbol{x}_{1}$ in the square area. Fig. 4(b) shows the norm of vector $\boldsymbol{v}_{\mathrm{c}}$, which corresponds to the square root of the nuclear norm of the matrix $\boldsymbol{\Xi}_{2,3}^{(2,3)}$, for different positions of $\boldsymbol{x}_{1}$ in the square area.

From these figures we can observe that the coupled information coupling when node 1 is far from neighbors. Moreover, the direction corresponding to the coupled information aligns with one of the directions of neighbors' cooperation when $\boldsymbol{v}_{1, i}$ and $\boldsymbol{v}_{2,3}$ are orthogonal for $i=2$ or $i=3$.

2) Scenario B: In this scenario, the position of node 2 at time step $t_{2}$ is $(1.66 \mathrm{~m}, 0 \mathrm{~m})$, the position of node 1 at time step $t_{1}$ is $(-1.66 \mathrm{~m}, 0 \mathrm{~m})$, and the position of node 2 at time step $t_{1}$ is $(0 \mathrm{~m},-5 \mathrm{~m})$. The matrices $\boldsymbol{K}_{i, i}=\boldsymbol{I}$ for $i \in\{1,2,3,4\}$, and the RIIs and SIIs $\lambda_{i, j}=50 / d_{i, j}^{2}$ for $i, j \in\{1,2,3,4\}$, where $d_{i, j}$ is the Euclidean distance between states $i$ and $j$. Fig. 5(a) shows the vectors $\boldsymbol{v}_{2 \rightarrow 1}, \boldsymbol{v}_{3 \rightarrow 1}$, and $\boldsymbol{v}_{\mathrm{c}}$ where $\boldsymbol{K}_{2 \rightarrow 1}^{(4,3)}=\boldsymbol{v}_{2 \rightarrow 1} \boldsymbol{v}_{2 \rightarrow 1}^{\mathrm{T}}, \boldsymbol{K}_{3 \rightarrow 1}^{(4,3)}=\boldsymbol{v}_{3 \rightarrow 1} \boldsymbol{v}_{3 \rightarrow 1}^{\mathrm{T}}$, and $\boldsymbol{\Xi}_{2,3}^{(4,3)}=\boldsymbol{v}_{\mathrm{c}} \boldsymbol{v}_{\mathrm{c}}^{\mathrm{T}}$ for a grid of 36 different positions of $\boldsymbol{x}_{1}$ in the square area. Fig. 5(b) shows the norm of vector $\boldsymbol{v}_{\mathrm{c}}$, which corresponds to the squared root of the nuclear norm of the matrix $\boldsymbol{\Xi}_{2,3}^{4,3}$, for different positions of $\boldsymbol{x}_{1}^{(2)}$ in the square area.

From these figures, we can observe that the coupled information decreases when the node 1 at time step $t_{2}$ is far from neighbors. Moreover, the coupled information also decreases when the direction formed by node 1 at times $t_{1}$ and $t_{2}$ is orthogonal to the direction formed by node 1 at time $t_{1}$ and node 2 at time $t_{1}$, that is, when the direction with neighbor $\boldsymbol{x}_{1}^{(1)}$ is orthogonal to the direction formed by the interaction set $\left\{\boldsymbol{x}_{1}^{(1)}, \boldsymbol{x}_{2}^{(1)}\right\}$. Conversely, the information coupling is more severe when the direction with neighbor $\boldsymbol{x}_{1}^{(1)}$ is the same as the interaction set and when node 1 at time $t_{2}$ is closer to such neighbor. Finally, the direction corresponding to the coupled information is closer to the direction of cooperation with node 


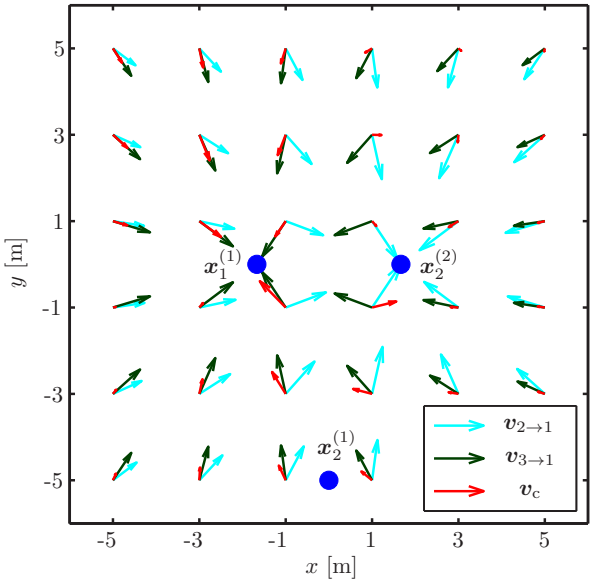

(a)

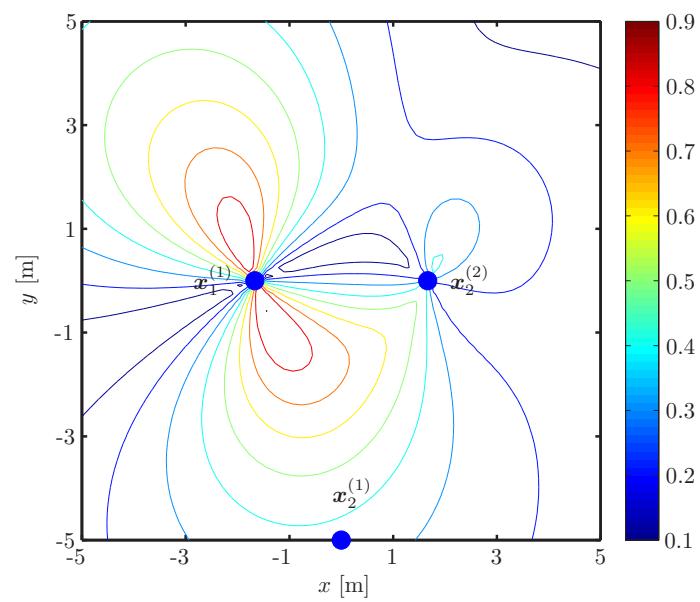

(b)

Fig. 5. Quantification of the coupled information for Scenario B. (a): Vectors $\boldsymbol{v}_{2 \rightarrow 1}, \boldsymbol{v}_{3 \rightarrow 1}$, and $\boldsymbol{v}_{\mathrm{c}}$ representing the effective information and the coupled information from cooperation with node 2 at time step $t_{2}$ and node 1 at time step $t_{1}$; (b): Intensity of information coupling for different values $\boldsymbol{x}_{1}$, measured by the norm of vector $\boldsymbol{v}_{\mathrm{c}}$ (nuclear norm of $\boldsymbol{\Xi}_{2,3}^{(4,3)}$ ).

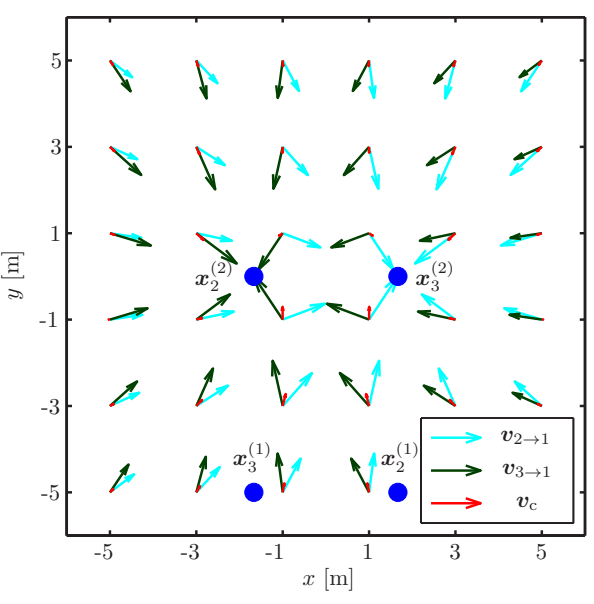

(a)

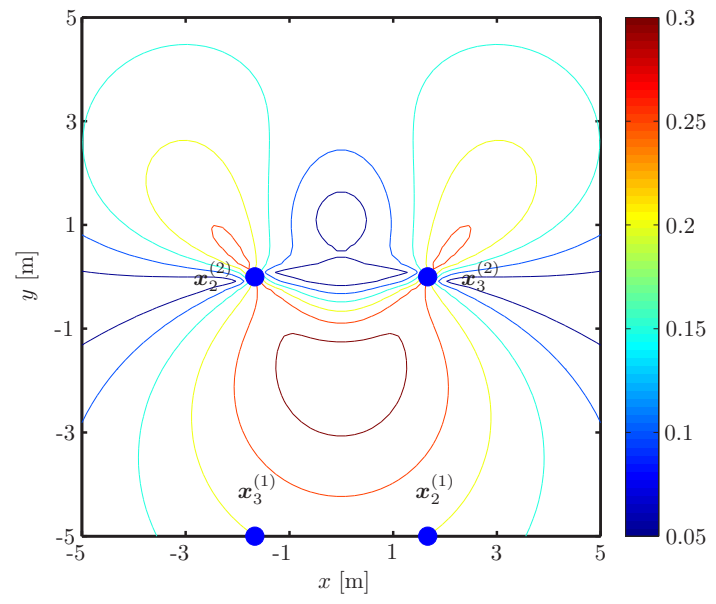

(b)

Fig. 6. Quantification of the coupled information for Scenario C. (a): Vectors $\boldsymbol{v}_{2 \rightarrow 1}, \boldsymbol{v}_{3 \rightarrow 1}$, and $\boldsymbol{v}_{\mathrm{c}}$ representing the effective information and the coupled information from cooperation with nodes 2 and 3 at time step $t_{2}$; (b): Intensity of information coupling for different values $\boldsymbol{x}_{1}^{(2)}$, measured by the norm of vector $\boldsymbol{v}_{\mathrm{c}}$ (nuclear norm of $\boldsymbol{\Xi}_{2,3}^{(4,5)}$ ).

1 at time $t_{1}$. These results agree with the intuition that the coupling directly affects neighbor $\boldsymbol{x}_{1}^{(1)}$ since this state is in the interaction set, and this effect comes mainly in the direction with the other state in the interaction set (node 2 at time $t_{1}$ ) while its effect is negligible in the orthogonal direction.

3) Scenario $C$ : In this scenario, the position of nodes 2 and 3 at time step $t_{2}$ are $(-1.66 \mathrm{~m}, 0 \mathrm{~m})$ and $(1.66 \mathrm{~m}, 0 \mathrm{~m})$, respectively, and the positions of nodes 2 and 3 at time step $t_{1}$ are $(1.66 \mathrm{~m},-5 \mathrm{~m})$ and $(-1.66 \mathrm{~m},-5 \mathrm{~m})$, respectively. The matrices $\boldsymbol{K}_{i, i}=\boldsymbol{I}$ for $i \in\{1,2,3,4,5\}$, and the RIIs and SIIs $\lambda_{i, j}=50 / d_{i, j}^{2}$ for $i, j \in\{1,2,3,4,5\}$, where $d_{i, j}$ is the Euclidean distance between states $i$ and $j$. Fig. 6(a) shows the vectors $\boldsymbol{v}_{2 \rightarrow 1}, \boldsymbol{v}_{3 \rightarrow 1}$, and $\boldsymbol{v}_{\mathrm{c}}$ where $\boldsymbol{K}_{2 \rightarrow 1}^{(4,5)}=\boldsymbol{v}_{2 \rightarrow 1} \boldsymbol{v}_{2 \rightarrow 1}^{\mathrm{T}}$, $\boldsymbol{K}_{3 \rightarrow 1}^{(4,5)}=\boldsymbol{v}_{3 \rightarrow 1} \boldsymbol{v}_{3 \rightarrow 1}^{\mathrm{T}}$, and $\boldsymbol{\Xi}_{2,3}^{(4,5)}=\boldsymbol{v}_{\mathrm{c}} \boldsymbol{v}_{\mathrm{c}}^{\mathrm{T}}$ for a grid of 36 different values of $\boldsymbol{x}_{1}^{(2)}$ in the square area. Fig. 6(b) shows the norm of vector $\boldsymbol{v}_{\mathrm{c}}$, which corresponds to the square root of the nuclear norm of the matrix $\boldsymbol{\Xi}_{2,3}^{(4,5)}$, for different positions of $\boldsymbol{x}_{1}^{(2)}$ in the square area.

From these figures we can observe that the information coupling is much less severe than in previous scenarios and decreases when the node 1 at time step $t_{2}$ is far from neighbors. Moreover, the coupled information also decreases when the direction formed by node 1 at time $t_{1}$ and each neighbor is orthogonal to the direction formed by each neighboring 


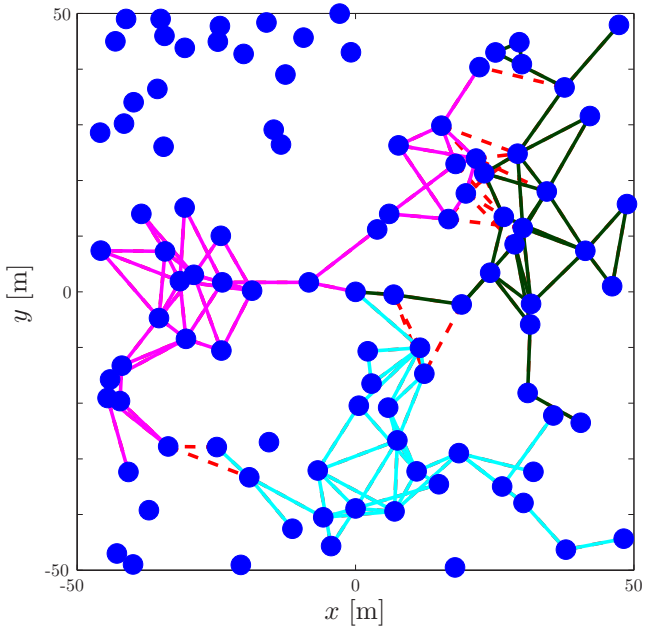

Fig. 7. Localization network formed by 100 nodes. Magenta, light blue, and dark green edges represent different connected components while red dashed edges represent an interaction set.

node with itself at previous time step. Conversely, the coupled information increases when the node 1 at time step $t_{2}$ is in the same direction as the direction formed by each neighboring node with itself, and hence the information coupling is more severe when node 1 at time step $t_{2}$ is in the intersection of both directions. These results agree with the intuition that firstly, the information coupling is much less severe because the coupling affects both neighbors indirectly (none of them are in the interaction set). In addition, the information coupling affects the neighbors in the direction they form with their neighbors in the interaction set, and hence, the information coupling affects node 1 at time step $t_{2}$ mainly in such directions while its effect is negligible in the orthogonal directions.

\section{B. Large Networks}

This section evaluates the intensity of information coupling over the total EFIM for large networks in terms of the nuclear norm of the corresponding matrices. In particular, we study how the closeness of the interaction set affects the coupled information. In addition, we show that close information links in terms of network distance are the truly relevant ones.

In this set of numerical simulations, we carried 10,000 Monte Carlo network emulations where 100 and 200 nodes are deployed uniformly at random in the square $[-50 \mathrm{~m}, 50 \mathrm{~m}] \times$ $[-50 \mathrm{~m}, 50 \mathrm{~m}]$ and the node $\iota$ is located in the origin (see Fig. 77. Each pair of nodes obtains range measurements with probability 0.5 if their distance is smaller than $16 \mathrm{~m}$ and the prior knowledge of nodes is set to $\boldsymbol{K}_{i, i}=\boldsymbol{I}, \boldsymbol{K}_{i, i}=4 \boldsymbol{I}$, or $\boldsymbol{K}_{i, i}=10 \boldsymbol{I}$.

In the first simulations, we study the relationship between information coupling and network distance of the interaction set. In these simulations the interaction set is formed by one link with different network distances to state $\iota$. In Fig. 8 we show the ratio between the nuclear norms of the coupled information $\boldsymbol{\Xi}$ and the total EFIM $\boldsymbol{J}_{\mathrm{e}}$ as a function of the network distance between the interaction set and state $\iota$ for networks formed by 100 and 200 nodes. In both figures, it can be observed that coupled information sharply decays with the network distance of the interaction set and also that information coupling is more severe in cases with small prior knowledge.

In the second simulations, we study how the information induced by a set of links decays with the network distance. In these simulations we compare the EFIM for state $\iota$ using all the information links $J_{\mathrm{e}}$ with that obtained using the information links between nodes with network distances at most $k, \boldsymbol{J}_{\mathrm{e}}^{\mathcal{B}_{k}}$, where $\mathcal{B}_{k}=\left\{(i, j) \in \mathcal{S}^{2}: \max \{\mathrm{ND}(\iota, i), \mathrm{ND}(\iota, j)\}>k\right\}$. Then, Fig. 9 shows the information intensities obtained from the nodes with network distances at most $k$ normalized by the total information intensity and the number of links to nodes with network distances at most $k$ for $k=1,2, \ldots, 10$. From this figure we can observe that the information intensity provided by each link in a network with network distances at most $k$ decays sharply with the network distance $k$.

\section{Information COUPLing AND Algorithms}

In network navigation, nodes obtain position estimates from spatial and temporal cooperation with neighbors. In previous sections, we have shown that the information an node obtains from its neighbors is often coupled since it depends not only on the information obtained from the links to each neighbor (e.g., links $\left(\iota, i_{1}\right)$ and $\left(\iota, i_{2}\right)$ in NIG shown in Fig. 1) but also on how those neighbors share information among themselves (e.g., link $\left(j_{1}, j_{2}\right)$ in NIG shown in Fig. 11). In this section, we show how such information coupling affects the performance of distributed algorithms and how our theoretical results can aid the design of such algorithms.

\section{A. Impact of Information Coupling on Algorithms}

In centralized algorithms, one processor collects all the information and determines jointly the positional estimates for all nodes. Such implementations can take full account of the information coupling since the centralized processor can have access to all pair-wise states' cooperations. That is, the processor can have complete access to the NIG formed by the pair-wise information links, and thus it can determine the coupled information for each state. For instance, in cases with linear and Gaussian models for measurements, a centralized processor can determine the posterior distribution of the states given all measurements as a joint Gaussian distribution. The mean of such distribution $\boldsymbol{\mu}$ represents the minimum meansquared error (MMSE) positional estimates for all states and the covariance matrix $\Sigma$ represents the joint uncertainty for all state estimates. In particular, the diagonal blocks in such joint covariance matrix represent the uncertainty of each state, while each non-diagonal block represents the correlation between pairs of states estimates. Note that a non-diagonal block $\boldsymbol{\Sigma}_{i, j}$ corresponding to states $i$ and $j$ is non-zero when there is path connecting states $i$ and $j$ in the NIG [79]. Such non-diagonal blocks quantify the interrelation between the estimates of states $i$ and $j$. 


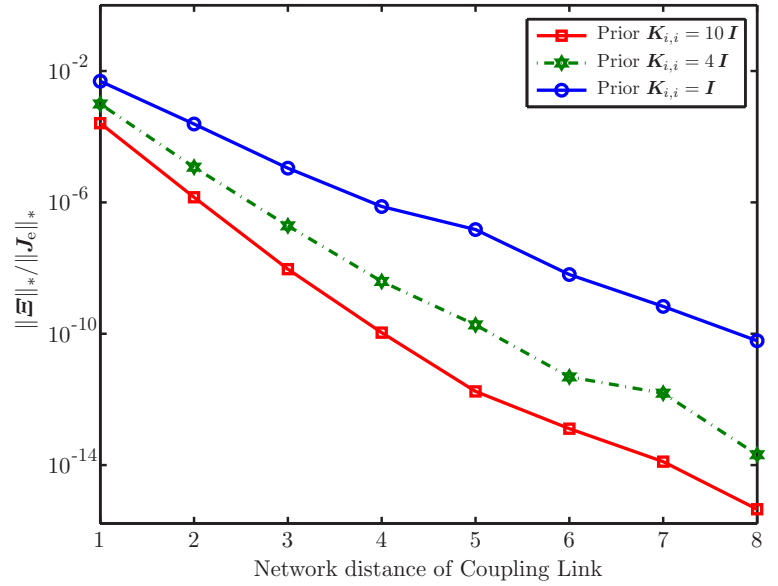

(a) Networks with 100 nodes.

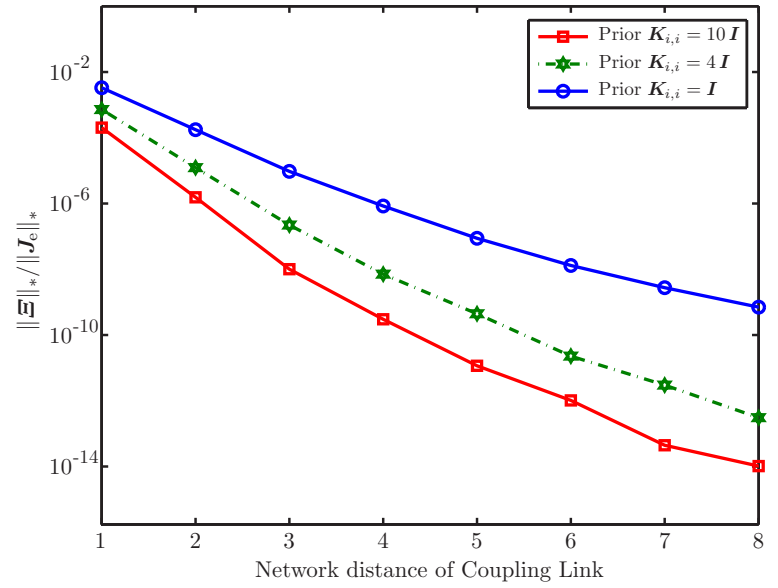

(b) Networks with 200 nodes.

Fig. 8. Coupled information sharply decays with the network distance of the interaction set and is more severe in cases with small prior knowledge. The $y$-axis represents averaged ratios between the nuclear norms of coupled information and the total equivalent information, while the $x$-axis represents different network distances of the interaction link.

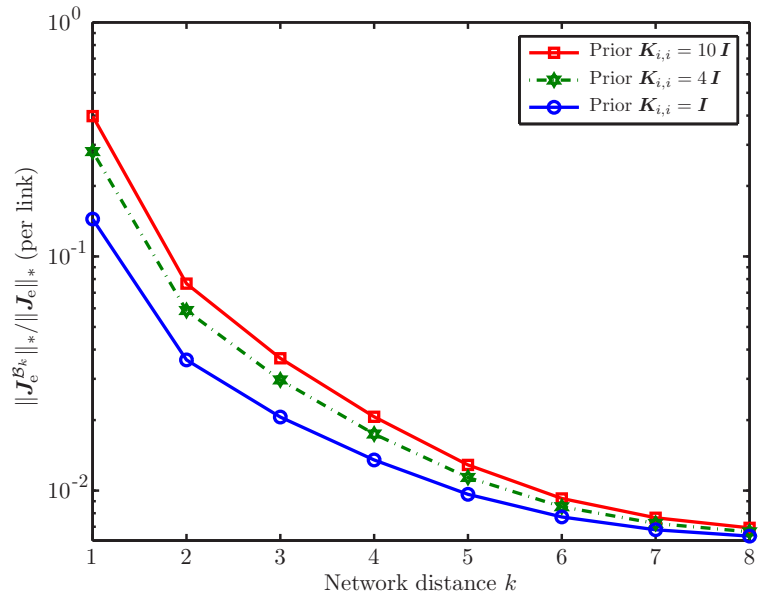

(a) Networks with 100 nodes.

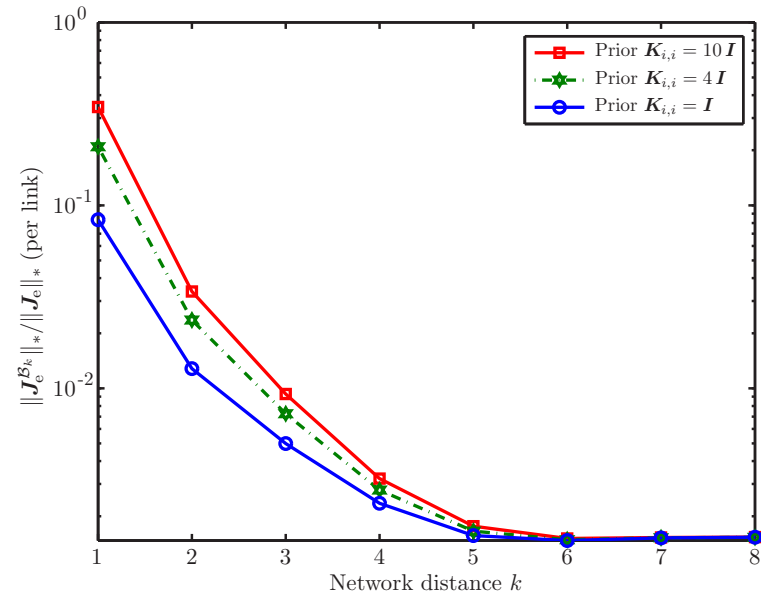

(b) Networks with 200 nodes.

Fig. 9. The impact of the links in the total information decays sharply with the network distance and increases with the amount of prior knowledge. The $y$-axis represents averaged nuclear norms of the EFIMs $\boldsymbol{J}_{\mathrm{e}}^{\mathcal{B}_{k}}$ obtained from nodes with network distances at most $k$ normalized by the total EFIM intensity and the number of links with network distances at most $k$, while the $x$-axis represents network distance $k$.

In distributed algorithms, several processors collect different subsets of information and determine in parallel positional estimates of subsets of nodes. In such implementations, it is difficult to account for information coupling since each processor has access to only a subset of pair-wise states cooperations. That is, each processor has access to a subgraph of the NIG formed by pair-wise information links. The most common current distributed implementations are based on belief propagation [51]-[53]. In these approaches, each processor keeps track of the positional estimate and uncertainty for each node separately, and individual estimates and uncertainties are updated by means of messages among different proces- sors. For instance, in cases with linear and Gaussian models for measurements, each processor approximates the marginal posterior of each individual state given all measurements as a Gaussian distribution. Each mean $\boldsymbol{\mu}_{i}$ of such distributions represents the positional estimate of each individual state and the corresponding covariance $\boldsymbol{\Sigma}_{i, i}$ represents the individual uncertainty of each state. Both means and covariances are updated through messages communicated with other processors through an iterative process. Note that those techniques are unaware of the interrelations among nodes' uncertainties and each processor does not keep track of how neighbors share information among themselves. 
In the following we show the impact of information coupling in the algorithms performance via both analytical expressions and numerical results using linear and Gaussian models for measurements for the ease of closed-form expressions. In particular, we consider a localization system that uses internode measurements related to the differences of positions and velocities, i.e., an inter-node measurement related to nodes $i$ and $j$ is $\boldsymbol{y}_{i, j}$ given by

$$
\boldsymbol{y}_{i, j}=\left[\begin{array}{c}
\boldsymbol{p}_{i}-\boldsymbol{p}_{j} \\
\boldsymbol{v}_{i}-\boldsymbol{v}_{j}
\end{array}\right]+\boldsymbol{\varepsilon}_{i, j}
$$

with $\boldsymbol{p}_{i} \in \mathbb{R}^{d}$ and $\boldsymbol{v}_{i} \in \mathbb{R}^{d}$ being position and velocity of node $i$, and $\boldsymbol{\varepsilon}_{i, j}$ being additive white noise following a zero-mean Gaussian distribution with covariance

$$
\boldsymbol{Q}_{i, j}=\operatorname{diag}\left\{\sigma_{\mathrm{p}}^{2} \boldsymbol{I}_{d}, \sigma_{\mathrm{v}}^{2} \boldsymbol{I}_{d}\right\}
$$

The state of node $k$ at time $t_{n}$ is $\boldsymbol{x}_{k}^{(n)}=\left[\left(\boldsymbol{p}_{k}^{(n)}\right)^{\mathrm{T}},\left(\boldsymbol{v}_{k}^{(n)}\right)^{\mathrm{T}}\right]^{\mathrm{T}} \in$ $\mathbb{R}^{2 d}$. Let $\boldsymbol{x}^{(n)}=\left[\left(\boldsymbol{x}_{1}^{(n)}\right)^{\mathrm{T}},\left(\boldsymbol{x}_{2}^{(n)}\right)^{\mathrm{T}}, \ldots,\left(\boldsymbol{x}_{K}^{(n)}\right)^{\mathrm{T}}\right]^{\mathrm{T}} \in$ $\mathbb{R}^{2 d K}$ be the concatenation of all states, and $\boldsymbol{y}^{(n)}=$ $\left[\left(\boldsymbol{y}_{i_{1}, j_{1}}^{(n)}\right)^{\mathrm{T}},\left(\boldsymbol{y}_{i_{2}, j_{2}}^{(n)}\right)^{\mathrm{T}}, \ldots,\left(\boldsymbol{y}_{i_{m}, j_{m}}^{(n)}\right)^{\mathrm{T}}\right] \in \mathbb{R}^{2 d M}$ be a set of internode measurements. Then, we have that

$$
\mathbf{y}^{(n)}=\boldsymbol{H}^{(n)} \boldsymbol{x}^{(n)}+\boldsymbol{\varepsilon}^{(n)}
$$

where

$$
\boldsymbol{H}^{(n)}=\left[\boldsymbol{e}_{i_{1}}^{K}-\boldsymbol{e}_{j_{1}}^{K}, \boldsymbol{e}_{i_{2}}^{K}-\boldsymbol{e}_{j_{2}}^{K}, \ldots, \boldsymbol{e}_{i_{m}}^{K}-\boldsymbol{e}_{j_{m}}^{K}\right]^{\mathrm{T}} \otimes \boldsymbol{I}_{2 d}
$$

with $\boldsymbol{\varepsilon}^{(n)}$ additive white noise that follows a zero-mean Gaussian distribution with covariance

$$
\boldsymbol{Q}^{(n)}=\operatorname{diag}\left\{\boldsymbol{Q}_{i_{1}, j_{1}}^{(n)}, \boldsymbol{Q}_{i_{2}, j_{2}}^{(n)}, \ldots, \boldsymbol{Q}_{i_{m}, j_{m}}^{(n)}\right\}
$$

We also assume that the nodes move with constant velocities, that is $\boldsymbol{x}_{k}^{(n)}=\boldsymbol{E} \boldsymbol{x}_{k}^{(n-1)}$ with

$$
\boldsymbol{E}=\left[\begin{array}{cc}
1 & \Delta \\
0 & 1
\end{array}\right] \otimes \boldsymbol{I}_{2 d}
$$

and $\Delta=t_{n}-t_{n-1}$ is a constant observation interval. Therefore, $\boldsymbol{x}^{(n)}=\boldsymbol{F} \boldsymbol{x}^{(n-1)}$ with $\boldsymbol{F}=\boldsymbol{I}_{K} \otimes \boldsymbol{E}$.

Under such assumptions, if $\boldsymbol{\mu}^{(n)}$ and $\boldsymbol{\Sigma}^{(n)}$ denote the mean and covariance of $\boldsymbol{x}^{(n)}$ given measurements $\left\{\boldsymbol{y}^{(1)}, \boldsymbol{y}^{(2)}, \ldots \boldsymbol{y}^{(n)}\right\}$, the usual Kalman filtering recursion (see e.g., [80]- $[86]$ ) give us that

$$
\begin{aligned}
& \boldsymbol{\mu}^{(n)}=\widetilde{\boldsymbol{\mu}}+\widetilde{\boldsymbol{\Sigma}}\left(\boldsymbol{H}^{(n)}\right)^{\mathrm{T}}\left(\boldsymbol{Q}^{(n)}+\boldsymbol{H}^{(n)} \widetilde{\boldsymbol{\Sigma}}\left(\boldsymbol{H}^{(n)}\right)^{\mathrm{T}}\right)^{-1} \boldsymbol{z} \\
& \boldsymbol{\Sigma}^{(n)}=\left(\widetilde{\boldsymbol{\Sigma}}^{-1}+\left(\boldsymbol{H}^{(n)}\right)^{\mathrm{T}}\left(\boldsymbol{Q}^{(n)}\right)^{-1} \boldsymbol{H}^{(n)}\right)^{-1}
\end{aligned}
$$

where

$$
\begin{aligned}
\widetilde{\boldsymbol{\mu}} & =\boldsymbol{F} \boldsymbol{\mu}^{(n-1)} \\
\widetilde{\boldsymbol{\Sigma}} & =\boldsymbol{F} \boldsymbol{\Sigma}^{(n-1)} \boldsymbol{F}^{\mathrm{T}} \\
\boldsymbol{z} & =\boldsymbol{y}^{(n)}-\boldsymbol{H}^{(n)} \widetilde{\boldsymbol{\mu}} .
\end{aligned}
$$

In the specific case where only one measurement is obtained at time $t_{n}$ (i.e., $\boldsymbol{y}^{(n)}=\boldsymbol{y}_{i, j}^{(n)}$ ), (73) and (74) lead to (see Appendix 凹

$$
\begin{aligned}
\boldsymbol{\mu}_{i}^{(n)}= & \boldsymbol{W}_{1}\left(\boldsymbol{W}_{1}+\boldsymbol{W}_{2}\right)^{-1} \boldsymbol{E} \boldsymbol{\mu}_{i}^{(n-1)} \\
& +\boldsymbol{W}_{2}\left(\boldsymbol{W}_{1}+\boldsymbol{W}_{2}\right)^{-1}\left(\boldsymbol{y}_{i, j}^{(n)}+\boldsymbol{E} \boldsymbol{\mu}_{j}^{(n-1)}\right) \\
\left(\boldsymbol{\Sigma}^{(n)}\right)_{i, i}= & \boldsymbol{W}_{1}\left(\boldsymbol{W}_{1}+\boldsymbol{W}_{2}\right)^{-1} \boldsymbol{E}\left[\boldsymbol{\Sigma}^{(n-1)}\right]_{i, i} \boldsymbol{E}^{\mathrm{T}} \\
& +\boldsymbol{W}_{2}\left(\boldsymbol{W}_{1}+\boldsymbol{W}_{2}\right)^{-1} \boldsymbol{E}\left[\boldsymbol{\Sigma}^{(n-1)}\right]_{j, i} \boldsymbol{E}^{\mathrm{T}}
\end{aligned}
$$

with

$$
\begin{aligned}
& \boldsymbol{W}_{1}=\boldsymbol{Q}_{i, j}^{(n)}+\boldsymbol{E}\left(\left[\boldsymbol{\Sigma}^{(n-1)}\right]_{j, j}-\left[\boldsymbol{\Sigma}^{(n-1)}\right]_{j, i}\right) \boldsymbol{E}^{\mathrm{T}} \\
& \boldsymbol{W}_{2}=\boldsymbol{E}\left(\left[\boldsymbol{\Sigma}^{(n-1)}\right]_{i, i}-\left[\boldsymbol{\Sigma}^{(n-1)}\right]_{i, j}\right) \boldsymbol{E}^{\mathrm{T}}
\end{aligned}
$$

In this simple case, at time $t_{n}$ node $i$ cooperates with two neighbors: node $i$ at time $t_{n-1}$ (temporal cooperation) and node $j$ at time $t_{n}$ (spatial cooperation). Both the state estimates and its covariance in (76) and (77) become the weighted sum of estimates and covariances due to the temporal cooperation and spatial cooperation. Note that both weighted sums depend on the uncertainty of the two neighbors but also on how those uncertainties are interrelated. If the neighbors have interacted, then the non-diagonal term $\left[\boldsymbol{\Sigma}^{(n-1)}\right]_{i, j}$ in the covariance matrix is non-zero. Discarding such information coupling leads to an inaccurate positional estimate in (76) and a wrong covariance in (77). Such covariances lead to a harmful cascade effect since they are used by other neighbors in next time steps.

We next quantify the localization performance loss due to discarding information coupling. In particular, we compare the position error of algorithms that keep only the individual uncertainties of each state with that of algorithms that keep the joint uncertainty of all the states. We simulate a navigation network composed of 100 nodes and three anchors deployed uniformly at random in the square $[-50 \mathrm{~m}, 50 \mathrm{~m}] \times[-50 \mathrm{~m}, 50 \mathrm{~m}]$. Nodes move with constant velocities with components sampled uniformly in the interval $[-1 \mathrm{~m} / \mathrm{s}, 1 \mathrm{~m} / \mathrm{s}]$. The states are estimated using inter-node measurements for positions and velocities differences as described in (67). In particular, in every second each pair of nodes $i, j$ obtain measurements with probability 0.5 if their distance $d_{i, j}$ is smaller than $20 \mathrm{~m}$, and $\sigma_{\mathrm{p}}^{2}=\sigma_{\mathrm{v}}^{2}=d_{i, j}^{2} / 50 \mathrm{~m}^{2}$. Positions are estimated using three algorithms:

1) a centralized algorithm that obtains MMSE estimates for the states using 73 and (74);

2) a distributed algorithm that estimates the states using BP (see e.g., [67]) 11

3) a centralized algorithm that estimates the states using (73) and (74) but discard the non-diagonal blocks in the covariance matrix.

These three algorithms are the most relevant for this evaluation since the first one is optimal in the mean-squared error (MSE) sense, the second one is the most common distributed algorithm, and the third one is the same as the optimal algorithm except that it discards the information coupling.

\footnotetext{
${ }^{11}$ We use four iterations for message passing as this is a common practice used for cooperative localization [51].
} 


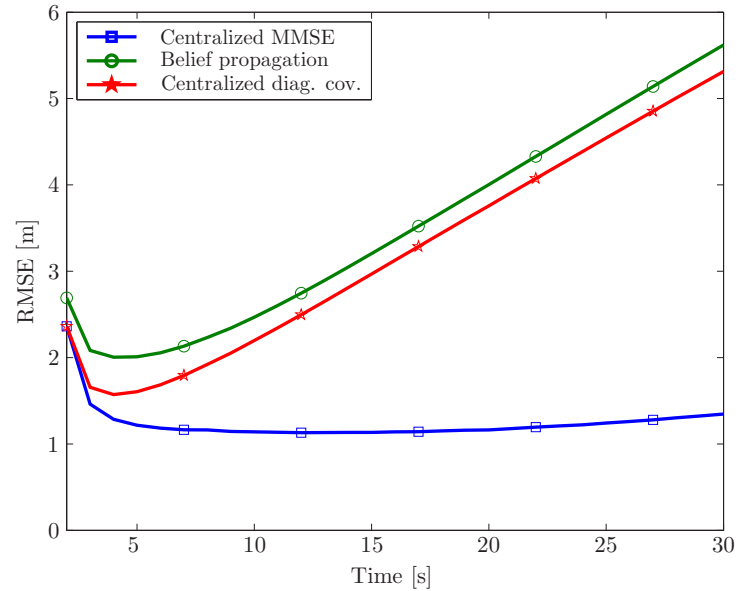

Fig. 10. The RMSE per node in a navigation network for centralized algorithm that estimates the states and joint nodes uncertainties versus a distributed algorithm based on BP, and a centralized algorithm that estimate the states and individual nodes' uncertainties. Position errors can rapidly increase if the information coupling among nodes' estimates are discarded.

Fig. 10 shows the root mean-squared error (RMSE) per node for the position estimates obtained by the three algorithms used during 30 seconds. It can be observed that the three algorithms obtain accurate position estimates for the first time steps, but that the algorithms that are not aware of the interrelations among estimates for different nodes (non-diagonal blocks in covariance matrix) quickly become highly inaccurate. In addition, Fig. 11] shows the RMSE ratio between the BP-based and MMSE algorithms for nodes with different number of neighbors. It can be observed that the performance gap between the BP-based and MMSE algorithms increases with the number of neighbors. As shown by these results, algorithms that discard the information coupling among different nodes can result in poor performances, specially in sequential implementations where the positions and uncertainties estimates are based on those obtained in previous steps.

\section{B. Design Insights Towards Coupling-aware Algorithms}

The paper characterizes the information coupling in network navigation via Fisher information analysis, and shows how the accuracy bound for each state depends on the effective information obtained from neighbors and also on the coupled information by neighbors' interaction. Specifically,

- Theorem 1 shows that the information obtained by each node through cooperation is the sum of that obtained from each connected component in the NIG. In addition, it shows that the information obtained from each component depends on the capacity of the links to the neighbors in such component and a term that accounts for the joint uncertainty of neighbors in the component. Corollary 1 shows that when neighbors are isolated, i.e., belong to different components, the information obtained through cooperation is the sum of the effective information obtained from each neighbor. Such effective information

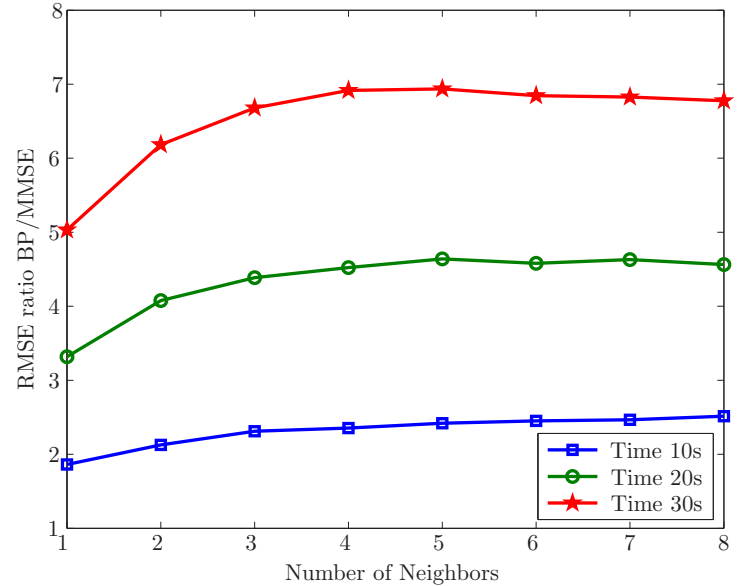

Fig. 11. Ratio between RMSEs of BP-based and MMSE algorithms for nodes with different number of neighbors. The performance gap of algorithms that discard information coupling increases with the number of neighbors.

accounts for the individual uncertainty of the neighbor in its component.

- Neighbors in the same connected component can be uncoupled by removing the information links in an interaction set. Proposition 2 shows that the information obtained through cooperation can be decoupled as the sum of the effective information from each neighbor by discarding the information links in an interaction set. Propositions 3 and 4 further characterize the effective information that can be obtained from neighbors in terms of the interaction set, neighbors uncertainty, and information links to neighbors.

- Theorem 2 with Corollaries 2 and 5 describes how coupled information decreases with the network distance to the interaction set. In particular Corollary 5 and the numerical results in Section $\mathrm{V}-\mathrm{B}$ show the sharp decay of coupled information with network distance. Such decay of coupled information implies that only close links induce a significant information coupling.

- Theorem 3 with Corollary 3 shows that the coupled information increases with the capacity of the link in the interaction set, and decreases with the uncertainty of the interaction set. In addition, the analytical expressions for specific simple representative networks shown in Section [V] and the corresponding numerical results shown in Section $\mathrm{V}$-A describe how information coupling also depends on the spatial topology among the states.

Our results show how interrelations among neighbors result in information coupling, how such coupling affects positional accuracy bounds, and the main aspects influencing information coupling. Several insights derived from such results can yield specially useful guidelines for the design of coupling-aware distributed algorithms.

- Algorithms that discard the information provided by interaction sets can directly lead to efficient distributed implementations. Such techniques can exploit a trade- 
off between positional accuracy and implementation efficiency where the information provided by interaction sets can be sacrificed in order to enable simple distributed implementations (see Propositions 2, 3, and 44).

- Algorithms that keep track of the most relevant information coupling can result in near optimal implementations. Our results show in what circumstances the information coupling is negligible depending on 1) the network distance to the interaction set (see Corollary 5 and Section $\mathrm{V}-\mathrm{B}$; 2) the interaction set uncertainties and link capacities (see Theorem 3 and Corollary 3); and 3) the spatial topology of neighbors and the interaction set (see Section V-A).

Efficient distributed estimation is critical not only for network navigation but also for a diverse set of applications in fields such as digital communications and sensor networks [87], [88]. Dependencies among different information sources hinder the development of effective distributed estimation techniques for general problems. For instance, BP-based distributed algorithms fail to take into account the dependences between messages from neighbors by assuming conditional independences, which are valid for tree-structured inference graphs but fail for graphs with loops. Capturing the dependencies among different information sources in distributed settings is challenging. Generalized BP techniques assemble variables in groups, and hence can keep the intra-group dependences but not inter-group dependences [88]. Other works suggest to keep track of the path followed by messages exchanged in oder to capture dependences between messages [87]. The theoretical results and design insights presented in this paper for network navigation can also be useful for general distributed estimation problems.

\section{CONCLUSION}

In this paper, we characterized the information coupling arising in network navigation via Fisher information analysis. In particular, we derived the EFIM for individual states, characterizing the effective information obtained from each neighbor and the coupled information induced by neighbors' interaction. In addition, we determined how the coupled information decays with network distance, analyzed information coupling in representative case studies, and showed the impact of information coupling in the performance of current distributed algorithms.

The results show that the information coupling depends on the links capacities, the node uncertainties, the spatiotemporal topology of the nodes, and the network distance of neighbors interaction. Information coupling can highly impact the performance of network navigation algorithms. The theoretical findings in the paper characterize how information coupling arises and which factors determine the intensity of information coupling. Our results can serve as guidelines for the design of efficient and accurate coupling-aware algorithms for network navigation that enable numerous emerging location-based applications.

\section{APPENDIX A}

PROOF OF PROPOSITION 1

Proof: The matrix $\boldsymbol{G}_{i, j}^{S} \otimes \boldsymbol{K}_{i, j}$ is positive semidefinite because $\boldsymbol{G}_{i, j}^{S} \otimes \boldsymbol{K}_{i, j}=\boldsymbol{C}_{i, j} \boldsymbol{K}_{i, j} \boldsymbol{C}_{i, j}^{\mathrm{T}}$ where $\boldsymbol{C}_{i, j}$ is a $S D \times D$ matrix given by

$$
\boldsymbol{C}_{i, j}= \begin{cases}\boldsymbol{e}_{i}^{S} \otimes \boldsymbol{I}_{D}, & i=j \\ \boldsymbol{e}_{i}^{S} \otimes \boldsymbol{I}_{D}-\boldsymbol{e}_{j}^{S} \otimes \boldsymbol{I}_{D}, & i \neq j .\end{cases}
$$

Then, the rank of $\boldsymbol{C}_{i, j} \boldsymbol{K}_{i, j} \boldsymbol{C}_{i, j}^{\mathrm{T}}$ is equal to that of $\boldsymbol{K}_{i, j}$ because $\boldsymbol{C}_{i, j}$ has rank $D$ which is larger than or equal to the rank of $\boldsymbol{K}_{i, j}$.

The second part of the proposition follows since using (1) we have that

$$
\boldsymbol{J} \succcurlyeq \sum_{i \in \mathcal{S}} \boldsymbol{G}_{i, i}^{S} \otimes \boldsymbol{K}_{i, i}
$$

where the right-hand side is full rank since it is block diagonal and $\boldsymbol{K}_{i, i} \in \mathbb{S}_{++}^{D}$ for all $i \in \mathcal{S}$.

\section{APPENDIX B LEMMA}

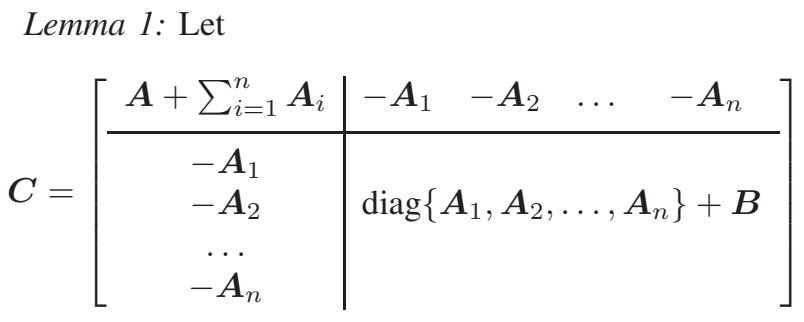

where $\boldsymbol{A}_{1}, \boldsymbol{A}_{2}, \ldots, \boldsymbol{A}_{n} \in \mathbb{S}_{+}^{D}, \boldsymbol{A} \in \mathbb{S}_{++}^{D}$, and $\boldsymbol{B} \in \mathbb{S}_{++}^{D n}$. Then, the matrix $C$ is positive definite and the Schur complement of $\boldsymbol{A}+\sum_{i=1}^{n} \boldsymbol{A}_{i}$ in $\boldsymbol{C}$ is $\boldsymbol{A}+\boldsymbol{R}$ with $\boldsymbol{R} \succcurlyeq 0$.

Proof: The matrix $C$ can be rewritten as

$$
\begin{aligned}
\boldsymbol{C}=\sum_{i=1}^{n}\left(\boldsymbol{e}_{1}^{n} \otimes \boldsymbol{U}_{i}-\boldsymbol{e}_{i}^{n} \otimes \boldsymbol{U}_{i}\right)\left(\boldsymbol{e}_{1}^{n} \otimes \boldsymbol{U}_{i}-\boldsymbol{e}_{i}^{n} \otimes \boldsymbol{U}_{i}\right)^{\mathrm{T}} \\
+\left[\begin{array}{cc}
\boldsymbol{A} & 0 \\
0 & \boldsymbol{B}
\end{array}\right]
\end{aligned}
$$

where $\boldsymbol{A}_{i}=\boldsymbol{U}_{i} \boldsymbol{U}_{i}^{\mathrm{T}}$. Then, $\boldsymbol{C}$ is positive definite because the first term in that decomposition is the sum of positive semidefinite matrices and the last term is a positive definite matrix.

Finally, the Schur complement of $\boldsymbol{A}+\sum_{i=1}^{n} \boldsymbol{A}_{i}$ in $\boldsymbol{C}$ is $\boldsymbol{A}+\boldsymbol{R}$ where $\boldsymbol{R}$ is the Schur complement of

$$
\sum_{i=1}^{n}\left(\boldsymbol{e}_{1}^{n} \otimes \boldsymbol{U}_{i}-\boldsymbol{e}_{i}^{n} \otimes \boldsymbol{U}_{i}\right)\left(\boldsymbol{e}_{1}^{n} \otimes \boldsymbol{U}_{i}-\boldsymbol{e}_{i}^{n} \otimes \boldsymbol{U}_{i}\right)^{\mathrm{T}}+\left[\begin{array}{cc}
0 & 0 \\
0 & \boldsymbol{B}
\end{array}\right]
$$

and hence, the result is obtained by observing that $\boldsymbol{R}$ is the Schur complement of a positive semidefinite matrix. 


\section{APPENDIX C \\ ProOf of Proposition 3}

Proof:

1) The inequality $\boldsymbol{K}_{i \rightarrow \iota}^{\mathcal{B}} \preccurlyeq \boldsymbol{K}_{\iota, i}$ is trivial since $\boldsymbol{\Lambda}_{k_{i}}^{\mathcal{B}}$ is a positive semidefinite matrix. The inequality $\boldsymbol{K}_{i \rightarrow \iota}^{\mathcal{B}^{\prime}} \succcurlyeq 0$ is obtained by observing that it is the Schur complement of

$$
\left[\begin{array}{cc}
\boldsymbol{K}_{\iota, i} & \boldsymbol{K}_{\iota, i} \\
\boldsymbol{K}_{\iota, i} & \left.\left(\left[\begin{array}{l}
\left(\boldsymbol{J}_{\mathcal{C}_{k_{i}}^{\prime}}^{\mathcal{B}^{\prime}}, \mathcal{C}_{k_{i}}^{\prime}\right.
\end{array}\right)^{-1}\right]_{i, i}\right)^{-1}
\end{array}\right]
$$

which is a positive semidefinite matrix since

$$
\left(\left[\left(\boldsymbol{J}_{\mathcal{C}_{k_{i}}^{\prime}, \mathcal{C}_{k_{i}}^{\prime}}^{\mathcal{B}^{\prime}}\right)^{-1}\right]_{i, i}\right)^{-1} \succcurlyeq \boldsymbol{K}_{\iota, i}
$$

using the Lemma1 with $\boldsymbol{C}=\boldsymbol{J}_{\mathcal{C}_{k_{i}}^{\prime}, \mathcal{C}_{k_{i}}^{\prime}}^{\mathcal{B}^{\prime}}$ and $\boldsymbol{A}=\boldsymbol{K}_{\iota, i}+$ $\boldsymbol{K}_{i, i}$. Finally, the inequality $\boldsymbol{K}_{i \rightarrow \iota}^{\mathcal{B}^{\prime}} \preccurlyeq \boldsymbol{K}_{i \rightarrow \iota}^{\mathcal{B}}$ is obtained by observing that $\boldsymbol{J}^{\mathcal{B}} \succcurlyeq \boldsymbol{J}^{\mathcal{B}^{\prime}}$.

2) The result is obtained by using the Lemma 1 with $C=$ $\boldsymbol{J}_{\mathcal{C}_{k_{i}}^{\prime}, \mathcal{C}_{k_{i}}^{\prime}}^{\mathcal{K}^{\prime}}$ and $\boldsymbol{A}=\boldsymbol{K}_{i, i}$. Notice that the result for $\mathcal{B}$ can be obtained analogously.

\section{APPENDIX D}

Proof OF THEOREM 2

Proof: The fact that $\left[\boldsymbol{J}_{k}\right]_{i, j}=\left[\boldsymbol{J}_{k}^{\mathcal{B}}\right]_{i, j}=\boldsymbol{J}_{i, j}$ for $(i, j) \notin \mathcal{E}_{k}^{2}$ is straightforward by the definition of $\mathcal{S}_{k}$ and $\mathcal{E}_{k}$. The remaining of the theorem can be proven by induction over $k$ as follows.

The result for $k=0$ is straightforward. Suppose the result holds for $k=r \geqslant 0$. Since $\left[\boldsymbol{J}_{r+1}\right]_{\mathcal{E}_{r+1}, \mathcal{E}_{r+1}}$ and $\left[\boldsymbol{J}_{r+1}^{\mathcal{B}}\right]_{\mathcal{E}_{r+1}, \mathcal{E}_{r+1}}$ are the Schur complement of the upper left block of

$$
\left[\begin{array}{cc}
\boldsymbol{J}_{\mathcal{E}_{r+1}, \mathcal{E}_{r+1}} & \boldsymbol{J}_{\mathcal{E}_{r+1}, \mathcal{E}_{r}} \\
\boldsymbol{J}_{\mathcal{E}_{r+1}^{\mathrm{T}}, \mathcal{E}_{r}} & {\left[\boldsymbol{J}_{r}\right]_{\mathcal{E}_{r}, \mathcal{E}_{r}}}
\end{array}\right] \text { and }\left[\begin{array}{cc}
\boldsymbol{J}_{\mathcal{E}_{r+1}, \mathcal{E}_{r+1}} & \boldsymbol{J}_{\mathcal{E}_{r+1}, \mathcal{E}_{r}} \\
\boldsymbol{J}_{\mathcal{E}_{r+1}}^{\mathrm{T}}, \mathcal{E}_{r} & {\left[\boldsymbol{J}_{r}^{\mathcal{B}}\right]_{\mathcal{E}_{r}, \mathcal{E}_{r}}}
\end{array}\right]
$$

respectively, then

$$
\begin{aligned}
& {\left[\boldsymbol{J}_{r+1}\right]_{\mathcal{E}_{r+1}, \mathcal{E}_{r+1}}-\left[\boldsymbol{J}_{r+1}^{\mathcal{B}}\right]_{\mathcal{E}_{r+1}, \mathcal{E}_{r+1}}} \\
& \quad=\boldsymbol{J}_{\mathcal{E}_{r+1}, \mathcal{E}_{r}}\left[\left(\left[\boldsymbol{J}_{r}^{\mathcal{B}}\right]_{\mathcal{E}_{r}, \mathcal{E}_{r}}\right)^{-1}-\left(\left[\boldsymbol{J}_{r}\right]_{\mathcal{E}_{r}, \mathcal{E}_{r}}\right)^{-1}\right] \boldsymbol{J}_{\mathcal{E}_{r+1}, \mathcal{E}_{r}}^{\mathrm{T}}
\end{aligned}
$$

and the result is obtained since by the induction hypothesis

$$
\begin{aligned}
{\left[\boldsymbol{J}_{r}\right]_{\mathcal{E}_{r}, \mathcal{E}_{r}=}=} & \left.\boldsymbol{J}_{r}^{\mathcal{B}}\right]_{\mathcal{E}_{r}, \mathcal{E}_{r}} \\
& +\boldsymbol{\Gamma}_{r} \boldsymbol{U}_{j_{1}, j_{2}}\left(\boldsymbol{I}+\boldsymbol{U}_{j_{1}, j_{2}}^{\mathrm{T}} \boldsymbol{\Delta}_{r} \boldsymbol{U}_{j_{1}, j_{2}}\right)^{-1} \boldsymbol{U}_{j_{1}, j_{2}}^{\mathrm{T}} \boldsymbol{\Gamma}_{r}^{\mathrm{T}}
\end{aligned}
$$

and hence

$$
\begin{aligned}
& \left(\left[\boldsymbol{J}_{r}^{\mathcal{B}}\right]_{\mathcal{E}_{r}, \mathcal{E}_{r}}\right)^{-1}-\left(\left[\boldsymbol{J}_{r}\right]_{\mathcal{E}_{r}, \mathcal{E}_{r}}\right)^{-1} \\
& =\left(\left[\boldsymbol{J}_{r}^{\mathcal{B}}\right]_{\mathcal{E}_{r}, \mathcal{E}_{r}}\right)^{-1} \boldsymbol{\Gamma}_{r} \boldsymbol{U}_{j_{1}, j_{2}} \\
& \quad \cdot\left[\boldsymbol{I}+\boldsymbol{U}_{j_{1}, j_{2}}^{\mathrm{T}}\left(\boldsymbol{\Delta}_{r}+\boldsymbol{\Gamma}_{r}^{\mathrm{T}}\left(\left[\boldsymbol{J}_{r}^{\mathcal{B}}\right]_{\mathcal{E}_{r}, \mathcal{E}_{r}}\right)^{-1} \boldsymbol{\Gamma}_{r}\right) \boldsymbol{U}_{j_{1}, j_{2}}\right]^{-1} \\
& \quad \cdot \boldsymbol{U}_{j_{1}, j_{2}}^{\mathrm{T}} \boldsymbol{\Gamma}_{r}^{\mathrm{T}}\left(\left[\boldsymbol{J}_{r}^{\mathcal{B}}\right]_{\mathcal{E}_{r}, \mathcal{E}_{r}}\right)^{-1}
\end{aligned}
$$

using the matrix inversion lemma.

\section{APPENDIX E}

PROOF OF THEOREM 3

Proof: Note that

$$
J_{\overline{\mathcal{S}}, \overline{\mathcal{S}}}=\boldsymbol{J}_{\overline{\mathcal{S}}, \overline{\mathcal{S}}}^{\mathcal{B}}+\boldsymbol{V} \boldsymbol{V}^{\mathrm{T}}
$$

where $\boldsymbol{V}=\left(\boldsymbol{e}_{j_{1}}^{S-1}-\boldsymbol{e}_{j_{2}}^{S-1}\right) \otimes \boldsymbol{U}_{j_{1}, j_{2}}$ and $\boldsymbol{K}_{j_{1}, j_{2}}=\boldsymbol{U}_{j_{1}, j_{2}} \boldsymbol{U}_{j_{1}, j_{2}}^{\mathrm{T}}$. Then, using the matrix inversion lemma

$$
\left(\boldsymbol{J}_{\overline{\mathcal{S}}, \overline{\mathcal{S}}}\right)^{-1}=\left(\boldsymbol{J}_{\overline{\mathcal{S}}, \overline{\mathcal{S}}}^{\mathcal{\mathcal { S }}}\right)^{-1}-\left(\boldsymbol{J}_{\overline{\mathcal{S}}, \overline{\mathcal{S}}}^{\mathcal{\mathcal { S }}}\right)^{-1} \boldsymbol{V} \boldsymbol{\Delta} \boldsymbol{V}^{\mathrm{T}}\left(\boldsymbol{J}_{\overline{\mathcal{S}}, \overline{\mathcal{S}}}^{\mathcal{\mathcal { S }}}\right)^{-1}
$$

where $\boldsymbol{\Delta}=\left(\boldsymbol{I}+\boldsymbol{V}^{\mathrm{T}}\left(\boldsymbol{J}_{\overline{\mathcal{S}}, \overline{\mathcal{S}}}^{\mathcal{B}}\right)^{-1} \boldsymbol{V}\right)^{-1}$. Since $\boldsymbol{J}_{\overline{\mathcal{S}}, \overline{\mathcal{S}}}^{\mathcal{B}}$ is block diagonal with each block corresponding to a different component of the partition of $\bar{S}$, all the components of $\left(\boldsymbol{J}_{\overline{\mathcal{S}}, \overline{\mathcal{S}}}\right)^{-1}$ and $\left(\boldsymbol{J}_{\overline{\mathcal{S}}, \overline{\mathcal{S}}}^{\mathcal{B}}\right)^{-1}$ are equal except for those corresponding to $\left(i_{1}, j_{1}\right)$, $\left(i_{1}, j_{2}\right),\left(i_{2}, j_{1}\right)$, and $\left(i_{2}, j_{2}\right)$ shown by 91, 92), and 93. respectively, at the bottom of the page.

Then the result is obtained since

$$
\begin{aligned}
\boldsymbol{U}_{j_{1}, j_{2}} \Delta \boldsymbol{U}_{j_{1}, j_{2}}^{\mathrm{T}} & =\boldsymbol{U}_{j_{1}, j_{2}}\left(\boldsymbol{I}+\boldsymbol{U}_{j_{1}, j_{2}}^{\mathrm{T}} \boldsymbol{\Phi} \boldsymbol{U}_{j_{1}, j_{2}}\right)^{-1} \boldsymbol{U}_{j_{1}, j_{2}}^{\mathrm{T}} \\
& =\left(\boldsymbol{I}-\boldsymbol{K}_{j_{1}, j_{2}}\left(\boldsymbol{\Phi}^{-1}+\boldsymbol{K}_{j_{1}, j_{2}}\right)^{-1}\right) \boldsymbol{K}_{j_{1}, j_{2}} \\
& =\boldsymbol{K}_{j_{1}, j_{2}}\left(\boldsymbol{\Phi}^{-1}+\boldsymbol{K}_{j_{1}, j_{2}}\right)^{-1} \boldsymbol{\Phi}^{-1}
\end{aligned}
$$

where the second equality is obtained using the matrix inversion lemma.

\section{APPENDIX F}

\section{Proof of Corollary 4}

Proof: Note that using (34) we have that

$$
\boldsymbol{\Xi}_{i_{1}, i_{2}}^{\left(j_{1}, j_{2}\right)}=\boldsymbol{\Gamma}\left(\boldsymbol{I}-\boldsymbol{K}_{j_{1}, j_{2}}\left(\boldsymbol{\Phi}^{-1}+\boldsymbol{K}_{j_{1}, j_{2}}\right)^{-1}\right) \boldsymbol{K}_{j_{1}, j_{2}} \boldsymbol{\Gamma}^{\mathrm{T}}
$$

and by the hypothesis of this corollary we have that

$$
\begin{aligned}
(\boldsymbol{I} & \left.-\boldsymbol{K}_{j_{1}, j_{2}}\left(\boldsymbol{\Phi}^{-1}+\boldsymbol{K}_{j_{1}, j_{2}}\right)^{-1}\right) \boldsymbol{K}_{j_{1}, j_{2}} \\
& =\left(1-\left(\boldsymbol{v}_{j_{1}, j_{2}}^{\mathrm{T}}\left(\boldsymbol{\Phi}^{-1}+\boldsymbol{v}_{j_{1}, j_{2}} \boldsymbol{v}_{j_{1}, j_{2}}^{\mathrm{T}}\right)^{-1}\right) \boldsymbol{v}_{j_{1}, j_{2}}\right) \boldsymbol{v}_{j_{1}, j_{2}} \boldsymbol{v}_{j_{1}, j_{2}}^{\mathrm{T}} \\
& =\left(1+\boldsymbol{v}_{j_{1}, j_{2}}^{\mathrm{T}} \boldsymbol{\Phi} \boldsymbol{v}_{j_{1}, j_{2}}\right)^{-1} \boldsymbol{v}_{j_{1}, j_{2}} \boldsymbol{v}_{j_{1}, j_{2}}^{\mathrm{T}} \\
& =\left(1+\boldsymbol{v}_{j_{1}, j_{2}}^{\mathrm{T}} \boldsymbol{\Phi} \boldsymbol{v}_{j_{1}, j_{2}}\right)^{-1} \boldsymbol{K}_{j_{1}, j_{2}}
\end{aligned}
$$

$$
\begin{aligned}
& {\left[\left(\boldsymbol{J}_{\overline{\mathcal{S}}, \overline{\mathcal{S}}}\right)^{-1}\right]_{i_{1}, j_{1}}=\left[\left(\boldsymbol{J}_{\mathcal{C}_{1}, \mathcal{C}_{1}}^{\mathcal{B}}\right)^{-1}\right]_{i_{1}, i_{1}}-\left[\left(\boldsymbol{J}_{\mathcal{C}_{1}, \mathcal{C}_{1}}^{\mathcal{B}}\right)^{-1}\right]_{i_{1}, j_{1}} \boldsymbol{U}_{j_{1}, j_{2}} \boldsymbol{\Delta} \boldsymbol{U}_{j_{1}, j_{2}}^{\mathrm{T}}\left[\left(\boldsymbol{J}_{\mathcal{C}_{1}, \mathcal{C}_{1}}^{\mathcal{B}}\right)^{-1}\right]_{i_{1}, j_{1}}} \\
& {\left[\left(\boldsymbol{J}_{\overline{\mathcal{S}}, \overline{\mathcal{S}}}\right)^{-1}\right]_{i_{1}, j_{2}}=\left(\left[\left(\boldsymbol{J}_{\overline{\mathcal{S}}, \overline{\mathcal{S}}}\right)^{-1}\right]_{i_{2}, j_{1}}\right)^{\mathrm{T}}=\left[\left(\boldsymbol{J}_{\mathcal{C}_{1}, \mathcal{C}_{1}}^{\mathcal{B}}\right)^{-1}\right]_{i_{1}, j_{1}} \boldsymbol{U}_{j_{1}, j_{2}} \boldsymbol{\Delta} \boldsymbol{U}_{j_{1}, j_{2}}^{\mathrm{T}}\left[\left(\boldsymbol{J}_{\mathcal{C}_{2}, \mathcal{C}_{2}}^{\mathcal{B}}\right)^{-1}\right]_{i_{2}, j_{2}}} \\
& {\left[\left(\boldsymbol{J}_{\overline{\mathcal{S}}, \overline{\mathcal{S}}}\right)^{-1}\right]_{i_{2}, j_{2}}=\left[\left(\boldsymbol{J}_{\mathcal{C}_{2}, \mathcal{C}_{2}}^{\mathcal{B}}\right)^{-1}\right]_{i_{2}, i_{2}}-\left[\left(\boldsymbol{J}_{\mathcal{C}_{2}, \mathcal{C}_{2}}^{\mathcal{B}}\right)^{-1}\right]_{i_{2}, j_{2}} \boldsymbol{U}_{j_{1}, j_{2}} \boldsymbol{\Delta} \boldsymbol{U}_{j_{1}, j_{2}}^{\mathrm{T}}\left[\left(\boldsymbol{J}_{\mathcal{C}_{2}, \mathcal{C}_{2}}\right)^{-1}\right]_{i_{2}, j_{2}}}
\end{aligned}
$$


where the second equality is a consequence of the matrix inversion lemma. Then, using the expression for $\Gamma$ in 35 , we have that

$$
\begin{aligned}
\boldsymbol{\Gamma} \boldsymbol{K}_{j_{1}, j_{2}}= & \left(\boldsymbol{v}_{\iota, i_{1}}^{\mathrm{T}}\left[\left(\boldsymbol{J}_{\mathcal{C}_{1}, \mathcal{C}_{1}}^{\mathcal{B}}\right)^{-1}\right]_{i_{1}, j_{1}} \boldsymbol{v}_{j_{1}, j_{2}} \boldsymbol{v}_{\iota, i_{1}}\right) \boldsymbol{v}_{j_{1}, j_{2}}^{\mathrm{T}} \\
& -\left(\boldsymbol{v}_{\iota, i_{2}}^{\mathrm{T}}\left[\left(\boldsymbol{J}_{\mathcal{C}_{2}, \mathcal{C}_{2}}^{\mathcal{B}}\right)^{-1}\right]_{i_{2}, j_{2}} \boldsymbol{v}_{j_{1}, j_{2}} \boldsymbol{v}_{\iota, i_{2}}\right) \boldsymbol{v}_{j_{1}, j_{2}}^{\mathrm{T}} \\
= & \left(\mu_{i_{1}} \boldsymbol{v}_{\iota, i_{1}}-\mu_{i_{2}} \boldsymbol{v}_{\iota, i_{2}}\right) \boldsymbol{v}_{j_{1}, j_{2}}^{\mathrm{T}}
\end{aligned}
$$

and the result is obtained observing that

$$
\boldsymbol{v}_{j_{1}, j_{2}}^{\mathrm{T}} \boldsymbol{\Gamma}^{\mathrm{T}}=\left(\mu_{i_{1}} \boldsymbol{v}_{\iota, i_{1}}-\mu_{i_{2}} \boldsymbol{v}_{\iota, i_{2}}\right)^{\mathrm{T}} .
$$

\section{APPENDIX G}

\section{DERIVATIONS FOR SECTIONS IV-A, IV-B, ANDIV-C}

The EFIM in (43) is obtained from Theorem 3 since in Scenario A, $i_{1}=j_{1}=2, i_{2}=j_{2}=3, \mathcal{C}_{1}=\{2\}$, and $\mathcal{C}_{2}=\{3\}$. Hence

$$
\begin{aligned}
& \left(\boldsymbol{J}_{\mathcal{C}_{1}, \mathcal{C}_{1}}^{\mathcal{B}}\right)^{-1}=\left(\boldsymbol{K}_{2,2}+\boldsymbol{K}_{1,2}\right)^{-1} \\
& \left(\boldsymbol{J}_{\mathcal{C}_{2}, \mathcal{C}_{2}}^{\mathcal{B}}\right)^{-1}=\left(\boldsymbol{K}_{3,3}+\boldsymbol{K}_{1,3}\right)^{-1}
\end{aligned}
$$

which leads to (43) using the expressions in Theorem 3 and (18).

The EFIM for the case with rank-one link capacities in 46 is obtained from Corollary 4 since from 18

$$
\begin{aligned}
\xi_{1, i} & =1-\boldsymbol{v}_{1, i}^{\mathrm{T}}\left(\boldsymbol{K}_{i, i}+\boldsymbol{K}_{1, i}\right)^{-1} \boldsymbol{v}_{1, i} \\
& =\left(1+\boldsymbol{v}_{1, i}^{\mathrm{T}} \boldsymbol{K}_{i, i}^{-1} \boldsymbol{v}_{1, i}\right)^{-1}
\end{aligned}
$$

using the matrix inversion lemma. The expression for $\mu_{i}$ is obtained since

$$
\boldsymbol{v}_{1, i}^{\mathrm{T}}\left(\boldsymbol{K}_{i, i}+\boldsymbol{K}_{1, i}\right)^{-1}=\xi_{1, i} \boldsymbol{v}_{1, i}^{\mathrm{T}} \boldsymbol{K}_{i, i}^{-1}
$$

using again the matrix inversion lemma. The expression for $\delta_{2,3}$ is a direct consequence of Corollary 4 and (43).

The EFIM in (50) is obtained from Theorem 3 since in Scenario $\mathrm{B}, i_{1}=2, j_{1}=4, i_{2}=j_{2}=3, \mathcal{C}_{1}=\{2,4\}$, and $\mathcal{C}_{2}=\{3\}$. Hence, $\left(\boldsymbol{J}_{\mathcal{C}_{1}, \mathcal{C}_{1}}^{\mathcal{B}}\right)^{-1}$ is given by 108 , shown at the bottom of the next page, and

$$
\left(\boldsymbol{J}_{\mathcal{C}_{2}, \mathcal{C}_{2}}^{\mathcal{B}}\right)^{-1}=\left(\boldsymbol{K}_{3,3}+\boldsymbol{K}_{1,3}\right)^{-1}
$$

which leads to (50) using the expressions in Theorem 3 and (18).

The EFIM for the case with rank-one link capacities in 53. is obtained from Corollary 4 The derivations for $\xi_{1,3}$ and $\mu_{2}$ are the same as those in Scenario A. The expression for $\delta_{3,4}$ is a direct consequence of Corollary 4 and (50), and $\xi_{1,2}$ is obtained since from (18) and the matrix inversion lemma

$$
\xi_{1,2}=1-\boldsymbol{v}_{1,2}^{\mathrm{T}} \boldsymbol{\Omega}_{1,2}^{-1} \boldsymbol{v}_{1,2}=\left(1+\boldsymbol{v}_{1,2}^{\mathrm{T}}\left(\boldsymbol{\Omega}_{2}-\boldsymbol{K}_{1,2}\right) \boldsymbol{v}_{1,2}\right)^{-1}
$$

and

$$
\begin{aligned}
\boldsymbol{\Omega}_{2}-\boldsymbol{K}_{1,2} & =\boldsymbol{K}_{2,2}+\boldsymbol{K}_{2,4}\left(\boldsymbol{K}_{4,4}+\boldsymbol{K}_{2,4}\right)^{-1} \boldsymbol{K}_{2,4} \\
& =\boldsymbol{K}_{2,2}+\left(1+\boldsymbol{v}_{2,4}^{\mathrm{T}} \boldsymbol{K}_{4,4}^{-1} \boldsymbol{v}_{2,4}\right)^{-1} \boldsymbol{K}_{2,4} .
\end{aligned}
$$

The expression for $\mu_{2}$ is obtained since from Corollary 4 and (43)

$$
\mu_{2}=\boldsymbol{v}_{1,2}^{\mathrm{T}} \boldsymbol{\Omega}_{2}^{-1} \boldsymbol{K}_{2,4}\left(\boldsymbol{K}_{4,4}+\boldsymbol{K}_{2,4}\right)^{-1} \boldsymbol{v}_{3,4}
$$

and using the matrix inversion lemma

$$
\begin{aligned}
& \boldsymbol{v}_{1,2}^{\mathrm{T}} \boldsymbol{\Omega}_{2}^{-1} \\
& \quad=\boldsymbol{v}_{1,2}^{\mathrm{T}}\left(\boldsymbol{\Omega}_{2}-\boldsymbol{K}_{1,2}\right)^{-1}\left(1+\boldsymbol{v}_{1,2}^{\mathrm{T}}\left(\boldsymbol{\Omega}_{2}-\boldsymbol{K}_{1,2}\right)^{-1} \boldsymbol{v}_{1,2}\right)^{-1} \\
& \quad=\xi_{1,2} \boldsymbol{v}_{1,2}^{\mathrm{T}}\left(\boldsymbol{\Omega}_{2}-\boldsymbol{K}_{1,2}\right)^{-1}
\end{aligned}
$$

and similarly

$\boldsymbol{K}_{2,4}\left(\boldsymbol{K}_{4,4}+\boldsymbol{K}_{2,4}\right)^{-1}=\boldsymbol{v}_{2,4} \boldsymbol{v}_{2,4}^{\mathrm{T}} \boldsymbol{K}_{4,4}^{-1}\left(1+\boldsymbol{v}_{2,4}^{\mathrm{T}} \boldsymbol{K}_{4,4}^{-1} \boldsymbol{v}_{2,4}\right)^{-1}$.

The EFIM in 57) is obtained from Theorem 3 since in Scenario $\mathrm{C}, i_{1}=2, j_{1}=4, i_{2}=3, j_{2}=5, \mathcal{C}_{1}=\{2,4\}$, and $\mathcal{C}_{2}=\{3,5\}$. Hence, $\left(\boldsymbol{J}_{\mathcal{C}_{1}, \mathcal{C}_{1}}^{\mathcal{B}}\right)^{-1}$ and $\left(\boldsymbol{J}_{\mathcal{C}_{2}, \mathcal{C}_{2}}^{\mathcal{B}}\right)^{-1}$ are given by (108) and (109), respectively, shown at the bottom of the page, which leads to (57) using the expressions in Theorem 3 and 18 .

The EFIM for the case with rank-one link capacities in (61) is obtained from Corollary 4. The derivations for $\xi_{1, i}$ and $\mu_{i}$ are the same as those of $\xi_{1,2}$ and $\mu_{2}$ in Scenario B. The expression for $\delta_{4,5}$ is a direct consequence of Corollary 4 and (57).

\section{APPENDIX H \\ PROOF OF COROLlaRY 5}

Proof: The proof is divided into two steps: we first prove that for $k=0,1, \ldots, n-1$,

$$
\boldsymbol{J}_{\mathcal{E}_{k}, \mathcal{E}_{k}}^{\mathcal{B}} \succcurlyeq \operatorname{diag}\left\{\left(\lambda_{i_{k}, i_{k}}+\lambda_{i_{k}, i_{k+1}}\right) \boldsymbol{I},\left(\lambda_{j_{k}, j_{k}}+\lambda_{j_{k}, j_{k+1}}\right) \boldsymbol{I}\right\}
$$

where we let $i_{n}=j_{n}=\iota$ for notational convenience. In the second step, we prove that for $k=0,1, \ldots, n-1, \boldsymbol{D}_{k} \preccurlyeq$ $2 \lambda_{i_{0}, j_{0}} \varepsilon^{k} \boldsymbol{I}$. Finally, the result is obtained by observing that $\boldsymbol{\Xi}^{\left(i_{0}, j_{0}\right)}=\boldsymbol{D}_{n} \preccurlyeq 4 \lambda_{i_{0}, j_{0}} \varepsilon^{n} \boldsymbol{I}$.

$$
\begin{aligned}
& \left(\boldsymbol{J}_{\mathcal{C}_{1}, \mathcal{C}_{1}}^{\mathcal{B}}\right)^{-1}=\left[\begin{array}{cc}
\boldsymbol{K}_{2,2}+\boldsymbol{K}_{1,2}+\boldsymbol{K}_{2,4} & -\boldsymbol{K}_{2,4} \\
-\boldsymbol{K}_{2,4} & \boldsymbol{K}_{4,4}+\boldsymbol{K}_{2,4}
\end{array}\right]^{-1}=\left[\begin{array}{cc}
\boldsymbol{\Omega}_{2}^{-1} & \boldsymbol{\Omega}_{2}^{-1} \boldsymbol{K}_{2,4}\left(\boldsymbol{K}_{4,4}+\boldsymbol{K}_{2,4}\right)^{-1} \\
\left(\boldsymbol{K}_{4,4}+\boldsymbol{K}_{2,4}\right)^{-1} \boldsymbol{K}_{2,4} \boldsymbol{\Omega}_{2}^{-1} & \boldsymbol{\Omega}_{4}^{-1}
\end{array}\right] \\
& \left(\boldsymbol{J}_{\mathcal{C}_{2}, \mathcal{C}_{2}}^{\mathcal{B}}\right)^{-1}=\left[\begin{array}{cc}
\boldsymbol{K}_{3,3}+\boldsymbol{K}_{1,3}+\boldsymbol{K}_{3,5} & -\boldsymbol{K}_{3,5} \\
-\boldsymbol{K}_{3,5} & \boldsymbol{K}_{5,5}+\boldsymbol{K}_{3,5}
\end{array}\right]^{-1}=\left[\begin{array}{cc}
\boldsymbol{\Omega}_{3}^{-1} & \boldsymbol{\Omega}_{3}^{-1} \boldsymbol{K}_{3,5}\left(\boldsymbol{K}_{5,5}+\boldsymbol{K}_{3,5}\right)^{-1} \\
\left(\boldsymbol{K}_{5,5}+\boldsymbol{K}_{3,5}\right)^{-1} \boldsymbol{K}_{3,5} \boldsymbol{\Omega}_{3}^{-1} & \boldsymbol{\Omega}_{5}^{-1}
\end{array}\right]
\end{aligned}
$$


First step: notice that

$$
\boldsymbol{J}_{\mathcal{E}_{0}, \mathcal{E}_{0}}^{\mathcal{B}}=\operatorname{diag}\left\{\left(\lambda_{i_{0}, i_{0}}+\lambda_{i_{0}, i_{1}}\right) \boldsymbol{I},\left(\lambda_{j_{0}, j_{0}}+\lambda_{j_{0}, j_{1}}\right) \boldsymbol{I}\right\}
$$

and then the result is obtained by induction since

$$
\begin{aligned}
& \boldsymbol{J}_{\mathcal{E}_{k+1}, \mathcal{E}_{k+1}}^{\mathcal{B}} \\
& =\left[\begin{array}{cc}
\left(\lambda_{i_{k+1}, i_{k+1}}+\lambda_{i_{k+1}, i_{k+2}}\right) \boldsymbol{I} & 0 \\
0 & \left(\lambda_{j_{k+1}, j_{k+1}}+\lambda_{j_{k+1}, j_{k+2}}\right) \boldsymbol{I}
\end{array}\right] \\
& +\left[\begin{array}{cc}
\lambda_{i_{k+1}, i_{k}} \boldsymbol{I} & 0 \\
0 & \lambda_{j_{k+1}, j_{k}} \boldsymbol{I}
\end{array}\right]-\left[\begin{array}{cc}
\lambda_{i_{k+1}, i_{k}} \boldsymbol{I} & 0 \\
0 & \lambda_{j_{k+1}, j_{k}} \boldsymbol{I}
\end{array}\right] \\
& \cdot\left(\boldsymbol{J}_{\mathcal{E}_{k}, \mathcal{E}_{k}}^{\mathcal{B}}\right)^{-1}\left[\begin{array}{cc}
\lambda_{i_{k+1}, i_{k}} \boldsymbol{I} & 0 \\
0 & \lambda_{j_{k+1}, j_{k}} \boldsymbol{I}
\end{array}\right]
\end{aligned}
$$

and hence, if the inequality in (110) is true for $k$ we have that it is also true for $k+1$ using (110) in (112) and since

$$
\begin{gathered}
\lambda_{i_{k+1}, i_{k}}-\frac{\lambda_{i_{k+1}, i_{k}}^{2}}{\lambda_{i_{k}, i_{k}}+\lambda_{i_{k+1}, i_{k}}}>0 \\
\lambda_{j_{k+1}, j_{k}}-\frac{\lambda_{j_{k+1}, j_{k}}^{2}}{\lambda_{j_{k}, j_{k}}+\lambda_{j_{k+1}, j_{k}}}>0 .
\end{gathered}
$$

Second step: notice that

$$
\boldsymbol{D}_{0}=\lambda_{i_{0}, j_{0}}[1,-1]^{\mathrm{T}}[1,-1] \otimes \boldsymbol{I} \preccurlyeq 2 \lambda_{i_{0}, j_{0}} \boldsymbol{I}
$$

and then $\boldsymbol{D}_{k} \preccurlyeq 2 \lambda_{i_{0}, j_{0}} \varepsilon^{k} \boldsymbol{I}$ for $k=0,1, \ldots, n-1$, by induction using Corollary 2 and the fact that

$$
\begin{array}{r}
\left(\boldsymbol{J}_{\mathcal{E}_{k}, \mathcal{E}_{k}}^{\mathcal{B}}\right)^{-2} \preccurlyeq \operatorname{diag}\left\{\left(\lambda_{i_{k}, i_{k}}+\lambda_{i_{k}, i_{k+1}}\right)^{-2} \boldsymbol{I},\right. \\
\left.\left(\lambda_{j_{k}, j_{k}}+\lambda_{j_{k}, j_{k+1}}\right)^{-2} \boldsymbol{I}\right\}
\end{array}
$$

and

$$
\begin{gathered}
\frac{\lambda_{i_{k+1}, i_{k}}}{\lambda_{i_{k}, i_{k}}+\lambda_{i_{k}, i_{k+1}}}<\frac{\lambda_{2}}{\lambda_{1}+\lambda_{2}} \\
\frac{\lambda_{j_{k+1}, j_{k}}}{\lambda_{j_{k}, j_{k}}+\lambda_{j_{k}, j_{k+1}}}<\frac{\lambda_{2}}{\lambda_{1}+\lambda_{2}} .
\end{gathered}
$$

Finally, from Corollary 2

$$
\begin{aligned}
\boldsymbol{D}_{n} & \preccurlyeq \boldsymbol{J}_{1, \mathcal{E}_{n-1}}\left(\boldsymbol{J}_{\mathcal{E}_{n-1}, \mathcal{E}_{n-1}}^{\mathcal{B}}\right)^{-1} \boldsymbol{D}_{n-1}\left(\boldsymbol{J}_{\mathcal{E}_{n-1}, \mathcal{E}_{n-1}}^{\mathcal{B}}\right)^{-1} \boldsymbol{J}_{\mathcal{E}_{n-1}, 1} \\
& \preccurlyeq 4 \lambda_{i_{0}, j_{0}} \varepsilon^{n} \boldsymbol{I}
\end{aligned}
$$

where the second inequality is obtained using (110) and $\boldsymbol{J}_{1, \mathcal{E}_{n-1}}=\left(\lambda_{1, i_{n-1}}, \lambda_{1, j_{n-1}}\right) \otimes \boldsymbol{I}=\boldsymbol{J}_{\mathcal{E}_{n-1}, 1}^{\mathrm{T}}$

\section{APPENDIX I}

DERIVATIONS FOR EQUATIONS (76) AND (77)

Since $\boldsymbol{y}^{(n)}=\boldsymbol{y}_{i, j}^{(n)}$, we have that $H^{(n)}=\left[\boldsymbol{e}_{i}^{K}-\boldsymbol{e}_{j}^{K}\right]^{\mathrm{T}} \otimes \boldsymbol{I}_{2 d}$ and hence

$$
\begin{array}{r}
\boldsymbol{z}=\boldsymbol{y}_{i, j}^{(n)}-\boldsymbol{E}\left(\boldsymbol{\mu}_{i}^{(n-1)}-\boldsymbol{\mu}_{j}^{(n-1)}\right) \\
\boldsymbol{Q}^{(n)}+\boldsymbol{H}^{(n)} \widetilde{\boldsymbol{\Sigma}}\left(\boldsymbol{H}^{(n)}\right)^{\mathrm{T}}=\boldsymbol{W}_{1}+\boldsymbol{W}_{2}
\end{array}
$$

with $\boldsymbol{W}_{1}$ and $\boldsymbol{W}_{2}$ given in (78). Therefore, using (73) we obtain

$$
\begin{aligned}
\boldsymbol{\mu}_{i}^{(n)}=\boldsymbol{E} \boldsymbol{\mu}_{i}^{(n-1)}+ & \boldsymbol{W}_{2}\left(\boldsymbol{W}_{1}+\boldsymbol{W}_{2}\right)^{-1} \\
& \cdot\left(\boldsymbol{y}_{i, j}^{(n)}-\boldsymbol{E}\left(\boldsymbol{\mu}_{i}^{(n-1)}-\boldsymbol{\mu}_{j}^{(n-1)}\right)\right)
\end{aligned}
$$

that directly leads to (76) after rearranging. Finally, (77) is obtained similarly after using the matrix inversion lemma in (74).

\section{ACKNOWLEDGMENT}

The authors would like to thank F. Meryer and H. Shin for their valuable suggestions and careful reading of the manuscript.

\section{REFERENCES}

[1] M. Z. Win, A. Conti, S. Mazuelas, Y. Shen, W. M. Gifford, D. Dardari, and M. Chiani, "Network localization and navigation via cooperation," IEEE Commun. Mag., vol. 49, no. 5, pp. 56-62, May 2011.

[2] Y. Shen, S. Mazuelas, and M. Z. Win, "Network navigation: Theory and interpretation," IEEE J. Sel. Areas Commun., vol. 30, no. 9, pp. 1823-1834, Oct. 2012.

[3] M. Z. Win, Y. Shen, and W. Dai, "A theoretical foundation of network localization and navigation," Proc. IEEE, vol. 106, no. 7, Jul. 2018, to appear.

[4] S. Gezici, Z. Tian, G. B. Giannakis, H. Kobayashi, A. F. Molisch, H. V. Poor, and Z. Sahinoglu, "Localization via ultra-wideband radios: A look at positioning aspects for future sensor networks," IEEE Signal Process. Mag., vol. 22, no. 4, pp. 70-84, Jul. 2005.

[5] A. H. Sayed, A. Tarighat, and N. Khajehnouri, "Network-based wireless location: Challenges faced in developing techniques for accurate wireless location information," IEEE Signal Process. Mag., vol. 22, no. 4, pp. 24-40, Jul. 2005

[6] K. Pahlavan, X. Li, and J.-P. Mäkelä, "Indoor geolocation science and technology," IEEE Commun. Mag., vol. 40, no. 2, pp. 112-118, Feb. 2002.

[7] J. J. Caffery and G. L. Stüber, "Overview of radiolocation in CDMA cellular systems," IEEE Commun. Mag., vol. 36, no. 4, pp. 38-45, Apr. 1998.

[8] M. Rabinowitz and J. J. Spilker, "A new positioning system using television synchronization signals," IEEE Trans. Broadcast., vol. 51, no. 1, pp. 51-61, March 2005.

[9] J. Warrior, E. McHenry, and K. McGee, "They know where you are location detection," IEEE Spectr., vol. 40, no. 7, pp. 20-25, 2003.

[10] J. Guivant, E. Nebot, and S. Baiker, "Autonomous navigation and map building using laser range sensors in outdoor applications," J. of Robotic Systems, vol. 17 , no. 10 , pp. 565-583, Oct. 2000

[11] N. Patwari, J. N. Ash, S. Kyperountas, A. O. Hero, R. L. Moses, and N. S. Correal, "Locating the nodes: Cooperative localization in wireless sensor networks," IEEE Signal Process. Mag., vol. 22, no. 4, pp. 54-69, Jul. 2005.

[12] K. Witrisal, P. Meissner, E. Leitinger, Y. Shen, C. Gustafson, F. Tufvesson, K. Haneda, D. Dardari, A. F. Molisch, A. Conti, and M. Z. Win, "High-accuracy localization for assisted living," IEEE Signal Process. Mag., vol. 33, no. 2, pp. 59-70, Mar. 2016.

[13] Z.-Q. Luo and W. Yu, "An introduction to convex optimization for communications and signal processing," IEEE J. Sel. Areas Commun., vol. 24, no. 8, pp. 1426-1438, Aug. 2006.

[14] I. F. Akyildiz, D. Pompili, and T. Melodia, "Underwater acoustic sensor networks: Research challenges," Ad Hoc Networks, vol. 3, no. 3, pp. 257-279, May 2005.

[15] R. Verdone, D. Dardari, G. Mazzini, and A. Conti, Wireless Sensor and Actuator Networks: Technologies, Analysis and Design. Amsterdam, The Netherlands: Elsevier, 2008.

[16] A. Conti, D. Dardari, and L. Zuari, "Cooperative UWB based positioning systems: CDAP algorithm and experimental results," in Proc. IEEE Int. Symp. on Spread Spectrum Tech. \& Applicat., Bologna, Italy, Aug. 2008 pp. 811-816.

[17] Y. Han, Y. Shen, X.-P. Zhang, M. Z. Win, and H. Meng, "Performance limits and geometric properties of array localization," IEEE Trans. Inf. Theory, vol. 62, no. 2, pp. 1054-1075, Feb. 2016.

[18] B. Denis, J.-B. Pierrot, and C. Abou-Rjeily, "Joint distributed synchronization and positioning in UWB ad hoc networks using TOA," IEEE Trans. Microw. Theory Techn., vol. 54, no. 4, pp. 1896 - 1911, Jun. 2006.

[19] T. Wang, Y. Shen, A. Conti, and M. Z. Win, "Network navigation with scheduling: Error evolution," IEEE Trans. Inf. Theory, vol. 63, no. 11, pp. 7509-7534, Nov. 2017.

[20] A. Rabbachin, I. Oppermann, and B. Denis, "GML ToA estimation based on low complexity UWB energy detection," in Proc. PIMRC '06, Helsinki, Finland, Sep. 2006.

[21] S. Bartoletti, A. Giorgetti, M. Z. Win, and A. Conti, "Blind selection of representative observations for sensor radar networks," IEEE Trans. Veh. Technol., vol. 64, no. 4, pp. 1388-1400, Apr. 2015. 
[22] F. Zabini and A. Conti, "Inhomogeneous Poisson sampling of finiteenergy signals with uncertainties in $\mathbb{R}^{d}, "$ IEEE Trans. Signal Process., vol. 64, no. 18, pp. 4679-4694, Sep. 2016.

[23] R. Niu, A. Vempaty, and P. Varshney, "Received signal strength based localization in wireless sensor networks," Proc. IEEE, vol. 106, no. 7, Jul. 2018.

[24] M. Z. Win, W. Dai, Y. Shen, G. Chrisikos, and H. V. Poor, "Network operation strategies for efficient localization and navigation," Proc. IEEE, vol. 106, no. 7, Jul. 2018, to appear.

[25] M. Chiani, A. Giorgetti, and E. Paolini, "Sensor radar for object tracking," Proc. IEEE, vol. 106, no. 6, Jun. 2018.

[26] Y. Xiong, N. Wu, Y. Shen, and M. Z. Win, "Cooperative network synchronization: Asymptotic analysis," IEEE Trans. Signal Process., vol. 66, no. 3, pp. 757-772, Feb. 2018.

[27] S. Mazuelas, R. M. Lorenzo, A. Bahillo, P. Fernandez, J. Prieto, and E. J. Abril, "Topology assessment provided by weighted barycentric parameters in harsh environment wireless location systems," IEEE Trans. Signal Process., vol. 58, no. 7, pp. 3842-3857, Jul. 2010.

[28] D. Dardari, A. Conti, U. J. Ferner, A. Giorgetti, and M. Z. Win, "Ranging with ultrawide bandwidth signals in multipath environments," Proc. IEEE, vol. 97, no. 2, pp. 404-426, Feb. 2009.

[29] J. Luo and Q. Zhang, "Relative distance based localization for mobile sensor networks," in Proc. IEEE Global Telecomm. Conf., Washington, DC, Nov. 2007, pp. 1076-1080.

[30] D. B. Jourdan, D. Dardari, and M. Z. Win, "Position error bound for UWB localization in dense cluttered environments," IEEE Trans. Aerosp. Electron. Syst., vol. 44, no. 2, pp. 613-628, Apr. 2008.

[31] L. Mailaender, "On the geolocation bounds for round-trip time-of-arrival and all non-line-of-sight channels," EURASIP J. Adv. in Signal Process., vol. 2008, pp. 1-10, 2008.

[32] Y. Shen and M. Z. Win, "Fundamental limits of wideband localization Part I: A general framework," IEEE Trans. Inf. Theory, vol. 56, no. 10, pp. 4956-4980, Oct. 2010.

[33] Y. Qi, H. Kobayashi, and H. Suda, "Analysis of wireless geolocation in a non-line-of-sight environment," IEEE Trans. Wireless Commun., vol. 5, no. 3, pp. 672-681, Mar. 2006.

[34] S. Bartoletti, W. Dai, A. Conti, and M. Z. Win, "A mathematical model for wideband ranging," IEEE J. Sel. Topics Signal Process., vol. 9, no. 2, pp. 216-228, Mar. 2015.

[35] J. J. Spilker, Jr., "GPS signal structure and performance characteristics," Journal of the Institute of Navigation, vol. 25, no. 2, pp. 121-146, Summer 1978.

[36] B. S. Pervan, D. G. Lawrence, and B. W. Parkinson, "Autonomous fault detection and removal using gps carrier phase," IEEE Trans. Aerosp. Electron. Syst., vol. 34, no. 3, pp. 897-906, Jul 1998.

[37] B. W. Parkinson, J. J. Spilker, P. Axelrad, and P. Enge, Eds., Global Positioning System: Theory and Applications, Volume I, ser. Progress in Astronautics and Aeronautics. Washington D.C., USA: AIAA, 1996.

[38] E. Foxlin, "Pedestrian tracking with shoe-mounted inertial sensors," IEEE Comput. Graph. Appl., vol. 25, no. 6, pp. 38-46, Nov.-Dec. 2005.

[39] S. Mazuelas, Y. Shen, and M. Z. Win, "Belief condensation filtering," IEEE Trans. Signal Process., vol. 61, no. 18, pp. 4403-4415, Sep. 2013

[40] G. Dissanayake, S. Sukkarieh, E. Nebot, and H. Durrant-Whyte, "The aiding of a low-cost strapdown inertial measurement unit using vehicle model constraints for land vehicle applications," IEEE Trans. Robot. Autom., vol. 17, no. 5, pp. 731 - 747, Oct. 2001.

[41] D. M. Bevly and B. Parkinson, "Cascaded kalman filters for accurate estimation of multiple biases, dead-reckoning navigation, and full state feedback control of ground vehicles," IEEE Trans. Control Syst. Technol., vol. 15, no. 2, pp. 199-208, March 2007.

[42] J. Prieto, S. Mazuelas, and M. Z. Win, "Context-aided inertial navigation via belief condensation," IEEE Trans. Signal Process., vol. 64, no. 12, pp. 3250-3261, Jun. 2016.

[43] W. Li, Y. Jia, and J. Du, "RSS-based joint detection and tracking in mixed LOS and NLOS environments," Digital Signal Processing, vol. 43, pp. 38 - 46, Aug. 2015.

[44] S. Maranò, W. M. Gifford, H. Wymeersch, and M. Z. Win, "NLOS identification and mitigation for localization based on UWB experimental data," IEEE J. Sel. Areas Commun., vol. 28, no. 7, pp. 1026-1035, Sep. 2010

[45] I. Güvenç, C.-C. Chong, F. Watanabe, and H. Inamura, "NLOS identification and weighted least-squares localization for UWB systems using multipath channel statistics," EURASIP J. Adv. in Signal Process., vol. 2008, pp. 1-14, Article ID 271 984, 2008.

[46] S. Mazuelas, A. Conti, J. C. Allen, and M. Z. Win, "Soft range information for network localization," IEEE Trans. Signal Process., vol. 66, no. 12, pp. 3155-3168, Jun. 2018.
[47] N. M. Freris, H. Kowshik, and P. R. Kumar, "Fundamentals of large sensor networks: Connectivity, capacity, clocks and computation," Proc. IEEE, vol. 98, no. 1, pp. 1828-1846, Nov. 2010.

[48] M. Dohler and Y. Li, Cooperative Communications: Hardware, Channel \& PHY. John Wiley \& Sons, 2010.

[49] M. R. Bhatnagar, A. Hjørungnes, and L. Song, "Cooperative communications over flat fading channels with carrier offsets: A doubledifferential modulation approach," EURASIP J. Adv. in Signal Process. vol. 2008, no. 531786, pp. 1-11, 2008 .

[50] H. Li, Z. Han, and H. V. Poor, "Asymptotic analysis of large cooperative relay networks using random matrix theory," EURASIP J. Adv. in Signal Process., vol. 2008, no. 235867, pp. 1-11, Apr. 2008.

[51] H. Wymeersch, J. Lien, and M. Z. Win, "Cooperative localization in wireless networks," Proc. IEEE, vol. 97, no. 2, pp. 427-450, Feb. 2009.

[52] A. T. Ihler, J. W. Fisher III, R. L. Moses, and A. S. Willsky, "Nonparametric belief propagation for self-localization of sensor networks," IEEE J. Sel. Areas Commun., vol. 23, no. 4, pp. 809-819, Apr. 2005.

[53] D. Fox, W. Burgard, H. Kruppa, and S. Thrun, "A probabilistic approach to collaborative multi-robot localization," Autonomous Robots, vol. 8, no. 3, pp. 325-344, Jun. 2000.

[54] C. Chang and A. Sahai, "Cramér-Rao-type bounds for localization," EURASIP J. Appl. Signal Process., vol. 2006, pp. Article ID 94287, 13 pages, 2006.

[55] Y. Shen, H. Wymeersch, and M. Z. Win, "Fundamental limits of wideband localization - Part II: Cooperative networks," IEEE Trans. Inf. Theory, vol. 56, no. 10, pp. 4981-5000, Oct. 2010.

[56] F. Yin, C. Fritsche, D. Jin, F. Gustafsson, and A. M. Zoubir, "Cooperative localization in WSNs using Gaussian mixture modeling: Distributed ECM algorithms," IEEE Trans. Signal Process., vol. 63, no. 6, pp. 1448 1463, Mar. 2015.

[57] W. Dai, Y. Shen, and M. Z. Win, "Distributed power allocation for cooperative wireless network localization," IEEE J. Sel. Areas Commun., vol. 33, no. 1, pp. 28-40, Jan. 2015.

[58] P. H. Tseng, Z. Ding, and K. T. Feng, "Cooperative self-navigation in a mixed LOS and NLOS environment," IEEE Trans. Mobile Comput., vol. 13, no. 2, pp. 350-363, Feb. 2014.

[59] X. Tan and J. Li, "Cooperative positioning in underwater sensor networks," IEEE Trans. Signal Process., vol. 58, no. 11, pp. 5860-5871, Nov. 2010.

[60] A. Conti, D. Dardari, M. Guerra, L. Mucchi, and M. Z. Win, "Experimental characterization of diversity navigation," IEEE Syst. J., vol. 8, no. 1, pp. 115-124, Mar. 2014.

[61] S. B. Cruz, T. E. Abrudan, Z. Xiao, N. Trigoni, and J. Barros, "Neighboraided localization in vehicular networks," IEEE Trans. Intell. Transp. Syst., vol. PP, no. 99, pp. 1-10, 2017.

[62] J. Yao, A. T. Balaei, M. Hassan, N. Alam, and A. G. Dempster, "Improving cooperative positioning for vehicular networks," IEEE Trans. Veh. Technol., vol. 60, no. 6, pp. 2810-2823, Jul. 2011.

[63] Y. Liu, Y. Shen, D. Guo, and M. Z. Win, "Network localization and synchronization using full-duplex radios," IEEE Trans. Signal Process., vol. 66, no. 3, pp. 714-728, Feb. 2018.

[64] U. A. Khan, S. Kar, and J. M. F. Moura, "DILAND: An algorithm for distributed sensor localization with noisy distance measurements," IEEE Trans. Signal Process., vol. 58, no. 3, pp. 1940-1947, Mar. 2010.

[65] — "Distributed sensor localization in random environments using minimal number of anchor nodes," IEEE Trans. Signal Process., vol. 57, no. 5, pp. 2000-2016, May 2009.

[66] S. Safavi, U. Khan, S. Kar, and J. Moura, "Distributed localization: A linear theory," Proc. IEEE, vol. 106, no. 7, Jul. 2018.

[67] Y. Weiss and W. T. Freeman, "Correctness of belief propagation in Gaussian graphical models of arbitrary topology," Neural Comput., vol. 13, no. 10, pp. 2173-2200, 2001.

[68] B. Zhou, Q. Chen, P. Xiao, and L. Zhao, "On the spatial error propagation characteristics of cooperative localization in wireless networks," IEEE Trans. Veh. Technol., vol. 66, no. 2, pp. 1647-1658, Feb. 2017.

[69] B. Zhou, Q. Chen, and P. Xiao, "The error propagation analysis of the received signal strength-based simultaneous localization and tracking in wireless sensor networks," IEEE Trans. Inf. Theory, vol. 63, no. 6, pp. 3983-4007, Jun. 2017.

[70] S. Mazuelas, Y. Shen, and M. Z. Win, "Information coupling in cooperative localization," IEEE Commun. Lett., vol. 15, no. 7, pp. 737739, Jul. 2011.

[71] - "Spatio-temporal information coupling in cooperative navigation," in Proc. IEEE Global Telecomm. Conf., Anaheim, CA, Dec. 2012, pp. 2403-2407. 
[72] A. Bahr, M. R. Walter, and J. J. Leonard, "Consistent cooperative localization," in Proc. IEEE Int. Conf. on Robotics and Automation, Kobe, Japan, May 2009, pp. 3415-3422.

[73] S. I. Roumeliotis and G. A. Bekey, "Synergetic localization for groups of mobile robots," in Proc. IEEE Conference on Decision and Control, Sydney, Australia, Dec. 2000, pp. 3477-3482.

[74] —_ "Distributed multirobot localization," IEEE Trans. Robot. Autom., vol. 18 , no. 5, pp. 781-795, Oct. 2002.

[75] H. L. Van Trees, Detection, Estimation and Modulation Theory, Part 1. New York, NY: Wiley, 1968.

[76] L. W. Beineke and R. J. Wilson, Topics in Algebraic Graph Theory. Cambridge University Press, 2004

[77] R. A. Horn and C. R. Johnson, Matrix Analysis, 1st ed. Cambridge, NY: Cambridge University Press, 1985.

[78] R. K. Ahuja, T. L. Magnanti, and J. B. Orlin, Network Flows: Theory, Algorithms, and Applications. Upper Saddle River, NJ, USA: PrenticeHall, 1993.

[79] B. Jones and M. West, "Covariance decomposition in undirected Gaussian graphical models," Biometrika, vol. 92, no. 4, pp. 779-786, Dec. 2005.

[80] H. V. Poor, An Introduction to Signal Detection and Estimation, 2nd ed. New York: Springer-Verlag, 1994.

[81] T. Kailath, Lectures on Wiener and Kalman filtering. Wien-New York: Springer-Verlag, 1981, second printing.

[82] _ "A view of three decades of linear filtering theory," IEEE Trans. Inf. Theory, vol. 20, no. 2, pp. 146-181, Mar. 1974.

[83] M. S. Branicky, V. S. Borkar, and S. K. Mitter, "A unified framework for hybrid control: Model and optimal control theory," IEEE Trans. Autom. Control, vol. 43, no. 1, pp. 31-45, Jan. 1998.

[84] S. K. Mitter, "Filtering and stochastic control: a historical perspective," IEEE Control Systems, vol. 16, no. 3, pp. 67-76, Jun. 1996

[85] M. Athans and P. L. Falb, Optimal Control: An Introduction to the Theory and Its Applications. New York: McGraw-Hill, 1966.

[86] P. L. Falb, "Infinite-dimensional filtering: The Kalman-Bucy filter in Hilbert space," Information and Control, vol. 11, no. 1-2, pp. 102-137, Jul.-Aug. 1967.

[87] M. Cetin, L. Chen, J. W. Fisher III, A. T. Ihler, R. L. Moses, M. J. Wainwright, and A. S. Willsky, "Distributed fusion in sensor networks," IEEE Signal Process. Mag., vol. 23, no. 4, pp. 42-55, Jul 2006.

[88] J. Yedidia, W. Freemand, and Y. Weiss, "Constructing free-energy approximations and generalized belief propagation algorithms," IEEE Trans. Inf. Theory, vol. 51, no. 7, pp. 2282-2312, July 2005.

Santiago Mazuelas (M'10-SM'17) received the Ph.D. in Mathematics and $\mathrm{Ph} . \mathrm{D}$ in Telecommunications Engineering from the University of Valladolid, Spain, in 2009 and 2011, respectively.

Since 2017 he has been Ramon y Cajal Researcher at the Basque Center for Applied Mathematics (BCAM). Prior to joining BCAM, he was a Staff Engineer at Qualcomm Corporate Research and Development from 2014 to 2017. He previously worked from 2009 to 2014 as Postdoctoral Fellow and Associate in the Laboratory for Information and Decision Systems (LIDS) at the Massachusetts Institute of Technology (MIT). His general research interest is the application of mathematics to solve engineering problems, currently his work is primarily focused on statistical signal processing, machine learning, and data science.

Dr. Mazuelas is Associate Editor for the IEEE Communications Letters and served as Co-chair for the Symposiums on Wireless Communications at the 2014 IEEE Globecom and at the 2015 IEEE ICC. He has received the Best Doctorate Thesis Award from University of Valladolid in 2011, the Young Scientist Prize from the Union Radio-Scientifique Internationale (URSI) Symposium in 2007, and the Early Achievement Award from the IEEE ComSoc in 2018. His papers received the IEEE Communications Society Fred W. Ellersick Prize in 2012, and Best Paper Awards from the IEEE ICC in 2013, the IEEE ICUWB in 2011, and the IEEE Globecom in 2011.
$\mathrm{He}$ is an Associate Professor with the Department of Electronic Engineering at Tsinghua University. Prior to that, he was a Research Assistant and then Postdoctoral Associate with the Wireless Information and Network Sciences Laboratory at MIT in 2005-2014. He was with the Hewlett-Packard Labs in winter 2009 and the Corporate R\&D at Qualcomm Inc. in summer 2008. His research interests include statistical inference, network science, communication theory, information theory, and optimization. His current research focuses on network localization and navigation, inference techniques, resource allocation, and intrinsic wireless secrecy.

Professor Shen was a recipient of the Qiu Shi Outstanding Young Scholar Award, the China's Youth 1000-Talent Program, the Marconi Society Paul Baran Young Scholar Award, and the MIT Walter A. Rosenblith Presidential Fellowship. His papers received the IEEE Communications Society Fred W. Ellersick Prize and three Best Paper Awards from the IEEE international conferences. He is elected Vice Chair (2017-2018) and Secretary (2015-2016) for the IEEE ComSoc Radio Communications Committee. He serves as TPC symposium Co-Chair for the IEEE Globecom (2018 and 2016), the European Signal Processing Conference (EUSIPCO) (2016), and the IEEE ICC Advanced Network Localization and Navigation (ANLN) Workshop (2016, 2017, and 2018). He also serves as Editor for the IEEE TRANSACTIONS ON WiRELESS COMMUNICATIONS since 2018, IEEE WIRLESS COMMUNICATIONS LETTERs since 2018, IEEE CHINA COMMUNICATIONS since 2017 , IEEE COMMUNICATIONS LETTERS (2015-2018), and Guest-Editor for the INTERNATIONAL JOURNAL OF DISTRIBUTED SENSOR NETWORKS (2015).

Moe Z. Win (S'85-M'87-SM'97-F'04) is a Professor at the Massachusetts Institute of Technology (MIT) and the founding director of the Wireless Information and Network Sciences Laboratory. Prior to joining MIT, he was with AT\&T Research Laboratories and NASA Jet Propulsion Laboratory.

His research encompasses fundamental theories, algorithm design, and network experimentation for a broad range of real-world problems. His current research topics include network localization and navigation, network interference exploitation, and quantum information science. He has served the IEEE Communications Society as an elected Member-at-Large on the Board of Governors, as elected Chair of the Radio Communications Committee, and as an IEEE Distinguished Lecturer. Over the last two decades, he held various editorial positions for IEEE journals and organized numerous international conferences. Currently, he is serving on the SIAM Diversity Advisory Committee.

Dr. Win is an elected Fellow of the AAAS, the IEEE, and the IET. He was honored with two IEEE Technical Field Awards: the IEEE Kiyo Tomiyasu Award (2011) and the IEEE Eric E. Sumner Award (2006, jointly with R. A. Scholtz). Together with students and colleagues, his papers have received numerous awards. Other recognitions include the IEEE Communications Society Edwin H. Armstrong Achievement Award (2016), the International Prize for Communications Cristoforo Colombo (2013), the Copernicus Fellowship (2011) and the Laurea Honoris Causa (2008) from the Università degli Studi di Ferrara, and the U.S. Presidential Early Career Award for Scientists and Engineers (2004). He is an ISI Highly Cited Researcher.
Yuan Shen (S'05-M'14) received the Ph.D. degree and the S.M. degree in electrical engineering and computer science from the Massachusetts Institute of Technology (MIT), Cambridge, MA, USA, in 2014 and 2008, respectively, and the B.E. degree (with highest honor) in electronic engineering from Tsinghua University, Beijing, China, in 2005. 